\title{
Integrable Background Geometries
}

\author{
David M.J. CALDERBANK
}

Department of Mathematical Sciences, University of Bath, Bath BA2 7AY, UK

E-mail: D.M.J.Calderbank@bath.ac.uk

URL: http://people.bath.ac.uk/dmjc20/

Received January 21, 2014, in final form March 18, 2014; Published online March 28, 2014

http://dx.doi.org/10.3842/SIGMA.2014.034

\begin{abstract}
This work has its origins in an attempt to describe systematically the integrable geometries and gauge theories in dimensions one to four related to twistor theory. In each such dimension, there is a nondegenerate integrable geometric structure, governed by a nonlinear integrable differential equation, and each solution of this equation determines a background geometry on which, for any Lie group $G$, an integrable gauge theory is defined. In four dimensions, the geometry is selfdual conformal geometry and the gauge theory is selfdual Yang-Mills theory, while the lower-dimensional structures are nondegenerate (i.e., nonnull) reductions of this. Any solution of the gauge theory on a $k$-dimensional geometry, such that the gauge group $H$ acts transitively on an $\ell$-manifold, determines a $(k+\ell)$-dimensional geometry $(k+\ell \leqslant 4)$ fibering over the $k$-dimensional geometry with $H$ as a structure group. In the case of an $\ell$-dimensional group $H$ acting on itself by the regular representation, all $(k+\ell)$-dimensional geometries with symmetry group $H$ are locally obtained in this way. This framework unifies and extends known results about dimensional reductions of selfdual conformal geometry and the selfdual Yang-Mills equation, and provides a rich supply of constructive methods. In one dimension, generalized Nahm equations provide a uniform description of four pole isomonodromic deformation problems, and may be related to the $\mathrm{SU}(\infty)$ Toda and dKP equations via a hodograph transformation. In two dimensions, the $\operatorname{Diff}\left(S^{1}\right)$ Hitchin equation is shown to be equivalent to the hyperCR Einstein-Weyl equation, while the $\operatorname{SDiff}\left(\Sigma^{2}\right)$ Hitchin equation leads to a Euclidean analogue of Plebanski's heavenly equations. In three and four dimensions, the constructions of this paper help to organize the huge range of examples of Einstein-Weyl and selfdual spaces in the literature, as well as providing some new ones. The nondegenerate reductions have a long ancestry. More recently, degenerate or null reductions have attracted increased interest. Two of these reductions and their gauge theories (arguably, the two most significant) are also described.
\end{abstract}

Key words: selfduality; gauge theory; twistor theory; integrable systems

2010 Mathematics Subject Classification: 53A30; 32L25; 37K25; 37K65; 53B35; 53C25; 58J70; 70S15; 83C20; 83C 80

\section{Introduction}

\section{Background}

This paper concerns differential geometry in dimensions one to four, and (primarily) four kinds of geometric structure, one in each dimension, governed by four nonlinear integrable differential equations. Associated to each manifold carrying one of these geometric structures, and to each Lie group, is an integrable gauge theory, generalizing a well-known gauge theory on flat space: the selfdual Yang-Mills equation on $\mathbb{R}^{4}$ or $\mathbb{R}^{2,2}$ and the gauge field equations arising as reductions by a (nondegenerate) group of translations to lower-dimensional flat spaces. Specifically, reduction by a single non-null translation gives the Bogomolny equation for monopoles on $\mathbb{R}^{3}$,

${ }^{\star}$ This paper is a contribution to the Special Issue on Progress in Twistor Theory. The full collection is available at http://www.emis.de/journals/SIGMA/twistors.html 
or its Lorentzian analogue on $\mathbb{R}^{2,1}[3,59]$. Reduction by two such translations yields the Hitchin equation for Higgs pairs on a Riemann surface, harmonic maps from a Riemann surface to a Lie group, or the principal chiral model on a two-dimensional space-time [30, 59]. Reduction by three non-null translations leads to the Nahm equation [44].

In addition to the physical motivation, the selfdual Yang-Mills equation has attracted interest because of its good integrability properties, which are inherited by the Bogomolny, Hitchin and Nahm equations, and their analogues. Further integrable systems may be obtained by reducing the selfdual Yang-Mills equation by other groups of conformal transformations of $\mathbb{R}^{4}$ or $\mathbb{R}^{2,2}$, and many such reductions have been investigated [41]. For example, hyperbolic monopoles arise from the reduction to three dimensions by a rotation, the Ernst equation is a reduction to two dimensions by a translation and rotation, while reductions to one dimension may be interpreted as isomonodromic deformation problems with four poles, governed (in the generic case) by the Schlesinger equation [31, 41, 43].

Twistor theory gives one explanation for this integrability: there is a Ward correspondence (see [5]) between solutions of the selfdual Yang-Mills equation on flat space and holomorphic vector bundles on (suitable open subsets of) $\mathbb{C P}^{3}$. This suggests that the selfdual Yang-Mills equation will continue to be integrable on other spaces $M$ so long as there is still a Ward correspondence between solutions and holomorphic vector bundles on some complex 3-manifold $Z$, the twistor space of $M$. Such curved twistor spaces $Z$ were introduced by Penrose [49] to study selfdual vacuum metrics. (Note that in Euclidean signature, selfdual Ricci-flat metrics are locally hyperkähler.)

Deep relationships between gauge field equations and selfdual vacuum metrics have been observed in a number of places. In [62], Ward considered gauge theories on $\mathbb{R}^{4-\ell}$ with the gauge group being a transitive group of diffeomorphisms of an $\ell$-manifold $\Sigma^{\ell}$. He focused on the group of diffeomorphisms preserving a fixed volume form on $\Sigma^{\ell}$ and observed that gauge fields then give rise to selfdual vacuum metrics on $\mathbb{R}^{4-\ell} \times \Sigma^{\ell}$. When $\ell=1$, the group of volume preserving diffeomorphisms of a circle or a line is $\mathrm{U}(1)$ or $\mathbb{R}$ acting by translation, so the monopoles are Abelian, and Ward's construction reduces to the Gibbons-Hawking Ansatz [24]. For $\ell=2$, finite-dimensional subgroups of $\operatorname{SDiff}\left(\Sigma^{2}\right)$ yield other interesting constructions of selfdual vacuum metrics [19], while $\ell=3$ gives the Ashtekar-Jacobson-Smolin description of selfdual vacuum metrics in terms of the Nahm equation [2]. One can also view the (closely related) Mason-Newman formulation [40] as the case $\ell=4$.

In Ward's construction, gauge fields on lower-dimensional flat spaces give rise to curved 4-manifolds. On the other hand, the example of hyperbolic monopoles shows that the lowerdimensional spaces can also be curved.

One of the goals of this paper is to place these miscellaneous results and observations in a geometric framework which simultaneously unifies and generalizes them. Selfdual vacuum metrics do not provide the right setting for this. For example, the natural generalization of the GibbonsHawking Ansatz to Abelian monopoles on hyperbolic space is LeBrun's hyperbolic Ansatz for scalar-flat Kähler metrics [39], and even on $\mathbb{R}^{4-\ell}$, one finds that without the volume-preserving condition, Ward's construction leads to hypercomplex, rather than hyperkähler, structures - see Hitchin [33] and Joyce [37] (or Dunajski [17]) for the analogue of the Ashtekar-Jacobson-Smolin and Mason-Newman description respectively.

In order to incorporate these constructions, it is essential to take into account a second basic feature of the selfdual Yang-Mills equation, in addition to integrability: conformal invariance. The selfdual Yang-Mills equation makes sense on any oriented conformal 4-manifold $M$, and the purely conformal part of Penrose's nonlinear graviton shows that there is a curved twistor space $Z$ as long as the conformal structure on $M$ is selfdual $[4,49]$.

The thesis of this paper is that reductions of selfdual conformal geometry to lower dimensions provide "integrable background geometries" on which curved versions of the Bogomolny, Hitchin 
and Nahm equations (with good integrability properties) can be defined. This thesis can be illustrated by the three-dimensional case: the Jones-Tod correspondence [36] shows that the reduction of a selfdual conformal structure to three dimensions is an Einstein-Weyl structure, and selfdual Yang-Mills fields reduce to generalized monopoles on such Einstein-Weyl spaces, placing Euclidean and hyperbolic monopoles in a common framework. Selfdual spaces with symmetry over a given Einstein-Weyl space are built out of Abelian monopoles, and this provides many constructions (generalizing the Gibbons-Hawking and hyperbolic Ansätze) of hyperkähler, scalarflat Kähler and selfdual Einstein metrics, and also of hypercomplex structures [10, 22, 29, 39].

The role of gauge fields and Abelian monopoles in Ward's construction and the Jones-Tod construction respectively suggests an underlying principle relating gauge field equations and constructions of selfdual spaces. This is amplified by the following two generalizations of the Jones-Tod construction.

First, in [8], the Jones-Tod construction and Ward's construction on $\mathbb{R}^{3}$ were simultaneously generalized by considering the Einstein-Weyl Bogomolny equation with the gauge group acting transitively by diffeomorphisms on a circle or a line. The volume preserving case gives the usual Jones-Tod correspondence between selfdual spaces with symmetry and Abelian monopoles on Einstein-Weyl spaces, but more general gauge groups lead to new constructions of selfdual conformal structures and metrics, including, in special cases, hyperkähler and selfdual Einstein metrics.

Second, note that the Jones-Tod construction gives rise to a procedure for constructing a new selfdual space from an invariant selfdual Maxwell field on a given selfdual space with one-dimensional symmetry group: the selfdual Maxwell field descends to an Abelian monopole on the quotient Einstein-Weyl space, out of which a new selfdual space may be built. This was generalized, by Maszczyk, Mason and Woodhouse [43], to any freely acting symmetry group, using a construction they call the "switch map" [41]: given a selfdual space with freely acting group of conformal transformations $H$, and an invariant selfdual Yang-Mills connection on a bundle $P$ with a gauge group $G$ of the same dimension as $H$, the quotient of $P$ by $H$ is another selfdual space, with symmetry group $G$. Hence, for example, $T^{3}$-invariant SU(2) YangMills fields on $\mathbb{R}^{4}$ give rise to selfdual conformal structures with $\mathrm{SU}(2)$ symmetry, such as the scalar-flat Kähler, hypercomplex and selfdual Einstein metrics of [14, 31, 33, 47, 55].

\section{Overview}

Ward's construction, the (generalized) Jones-Tod correspondence, and the switch map all point to the following framework for dimensional reduction of selfdual conformal geometry and the selfdual Yang-Mills equation.

(i) There are background geometries in each dimension less than four obtained by dimensional reduction of the selfduality condition for conformal structures.

(ii) The nonlinear differential equations defining these background geometries have the surprising feature that they do not depend on the symmetry group that one reduces by. Therefore, including selfdual conformal geometry, there are only four kinds of (nondegenerate) background geometry.

(iii) Instead, the symmetry group enters as a gauge group for a gauge theory defined on the background geometry, and the gauge field equation is the dimensional reduction of the selfdual Yang-Mills equation. Hence the gauge field equations play a remarkable dual role: solutions give rise both to selfdual conformal 4-manifolds and also to selfdual YangMills fields on such manifolds.

In this paper, the above framework is established and studied in full generality. Furthermore, the different geometries are related not just by symmetry reduction, but by a more general 
form of dimensional reduction, of which Ward's construction and the generalized Jones-Tod construction are examples (cf. also [25]). Such constructions also relate the lower-dimensional geometries to each other. More precisely:

- For $1 \leqslant k<k+\ell \leqslant 4,(k+\ell)$-dimensional geometries are obtained from a $k$-dimensional geometry by solving the gauge field equation on that background where the gauge group acts transitively by diffeomorphisms on an $\ell$-manifold.

In four dimensions, the gauge fields and background geometries are, of course, selfdual YangMills fields on selfdual spaces, while in three dimensions, one obtains monopoles on EinsteinWeyl spaces. The one and two-dimensional stories are new, although these structures have been implicitly studied in many places, at least in special cases $[28,51]$ and the twistor theory of the two-dimensional geometry has been developed independently by Donaldson and Fine [66, 76]. I first describe briefly the two-dimensional geometries, in the form obtained by reduction from Euclidean signature. The two reductions from Kleinian signature $(2,2)$ are similar.

Given a complex line bundle $\mathcal{W}$ over a Riemann surface $N$ with a Hermitian metric on $\mathcal{W}^{*} \otimes T N$, the geometric structure is a triple consisting of a $\mathrm{U}(1)$-connection $a$ on $\mathcal{W}^{*} \otimes T N$, a section $\psi$ of $\mathcal{W}^{*}$ and a section $\mathcal{C}$ of $\mathcal{W}^{*} \otimes \mathcal{W}^{*} \otimes T N$ satisfying the following equations:

$$
\bar{\partial}^{a} \mathcal{C}=0, \quad \bar{\partial}^{a} \psi=-3 \mathcal{C} \bar{\psi}, \quad * F^{a}=|\psi|^{2}-2|\mathcal{C}|^{2} .
$$

The flat geometry is obtained by setting $\mathcal{W}=T N$, with the trivial connection on $\mathcal{W}^{*} \otimes T N$ and $\mathcal{C}=\psi=0$. This is simply a Riemann surface with no additional structure. The general two-dimensional background geometry was found in joint work with Lionel Mason [9].

The gauge fields on this background, with gauge group $G$, are pairs $(A, \Phi)$ consisting of a $G$-connection $A$ and a section $\Phi$ of $\mathcal{W}^{*} \otimes \mathfrak{g}_{N}$, where $\mathfrak{g}_{N}$ is the associated Lie algebra bundle. These pairs satisfy the following equations:

$$
F^{A}-[\Phi, \bar{\Phi}]=\psi \wedge \bar{\Phi}+\bar{\psi} \wedge \Phi, \quad \bar{\partial}^{a, A} \Phi=\mathcal{C} \bar{\Phi} .
$$

On the flat geometry, where $\mathcal{C}=0=\psi$, these are the Hitchin equations for stable pairs, but in the general case, the equations are coupled to the background geometry.

In one dimension, the background geometry is governed by a symmetric traceless $(3 \times 3)$ matrix $\mathcal{B}$ satisfying the Riccati equation $\mathcal{B}_{r}=2\left(\mathcal{B}^{2}\right)_{0}$, where $r$ is an affine coordinate and the subscript zero denotes the traceless part. More invariantly, $\mathcal{B}$ is a section of $T^{*} C \otimes \operatorname{Sym}_{0} \mathcal{E}$ where $\mathcal{E}$ is a rank 3 conformal vector bundle over a curve $C$, with $\wedge^{3} \mathcal{E}=(T C)^{3}$, and one fixes compatible connections on $\mathcal{E}$ and $T C$ to define the $r$-derivative $\mathcal{B}_{r}$.

The gauge fields on this background are sections $\Phi$ of $\mathcal{E}^{*} \otimes \mathfrak{g}_{C}$ (where $\mathfrak{g}_{C}$ is a Lie algebra bundle over $C$ ) satisfying the equation $\Phi_{r}-*[\Phi, \Phi]=\mathcal{B} \cdot \Phi$, where $*$ denotes the star operator on $\mathcal{E}$ and one fixes a $G$-connection to define $\Phi_{r}$. The flat geometry is the trivial solution $\mathcal{B}=0$, in which case this gauge field equation reduces to the Nahm equation.

There is one further important property of the Jones-Tod correspondence that continues to hold in the general framework: it is constructive, i.e., a selfdual conformal structure is explicitly determined by an Einstein-Weyl structure together with a monopole, and conversely. The correspondence between additional monopoles and selfdual Yang-Mills fields is equally explicit. The same remarks hold for the switch map and Ward's construction. I therefore present explicit formulae for the constructions of the paper.

For convenience of exposition, I will concentrate on the reductions from Euclidean signature and the notation will be adapted to this case. However, the non-null reductions from Kleinian signature $(2,2)$ are completely analogous, as are such reductions of complex geometries: I indicate throughout the nondegeneracy assumptions that need to be made, and any changes in notation that are needed. On the other hand, it remains an interesting open project to study the integrable background geometries arising from null reductions: only two such reductions are considered here. 


\section{User guide}

This is a long paper to read from start to finish, so I give a detailed guide to the sections, both to draw attention to the highlights and to enable the reader to dip into the paper more easily.

The general theory of the paper is developed in Sections 2, 4, 6 and 8. Section 2 concerns selfdual spaces with a freely acting symmetry group and presents the background geometry equations in dimensions $1-3$. In each case the main result, Theorems $2.3,2.6$ or 2.9, identifies the selfduality condition on a conformal structure with the background geometry equations: in particular Theorem 2.9 is the Jones-Tod correspondence [36]. Section 4 deals with invariant selfdual Yang-Mills fields on selfdual spaces with a freely acting symmetry group. Here the gauge field equations on the background geometries are computed.

The main general theorems are in Sections 6 and 8, where the inverse constructions to Section 2 are established and generalized. Theorem 6.1 proves that selfdual spaces may be constructed from solutions to gauge field equations on $k$-dimensional background geometries where the gauge group acts transitively on a $(4-k)$-manifold: in the case of a group acting on itself by the regular representation, this theorem reconstructs the selfdual spaces with freely acting symmetry group of Section 2 - although, as mentioned already, in the general case, the group need not even be finite-dimensional.

Theorem 8.1 generalizes all this to gauge groups acting on $\ell$-manifolds, giving explicit constructions of $(k+\ell)$-dimensional background geometries from gauge fields on $k$-dimensional geometries for $k+\ell \leqslant 4$. Unfortunately, the calculations here are too complicated to present in full. However, I do provide explicit formulae: in examples arising in practice, it is usually not too hard to verify that the $(k+\ell)$-dimensional geometry satisfies the background equations, once one has a formula.

The relation between selfdual spaces, and the three-dimensional background geometries, Einstein-Weyl spaces, is a longstanding one, and as a consequence the latter have been extensively studied $[8,10,11,21,22,29,36,39,55,56,60]$. By contrast, the background geometries in one and two dimensions, Riccati spaces, and spinor-vortex spaces, although implicitly underlying previous work, have only been introduced and investigated relatively recently (see, e.g., [9, 66]). Assuming that one is not interested in zero-dimensional differential geometry (see Remark 7.9), then these geometries form the foundation for the more well-known higher-dimensional structures (using Theorems 6.1 and 8.1).

In Section 10, the geometry of Riccati spaces is described. Although there are only six Riccati spaces up to local isomorphism, they have a rather rich structure, which is most easily revealed in a complexified setting, since only three of the Riccati spaces arise as reductions from Euclidean signature. The six solutions correspond to the six types of quartic polynomial, i.e., the five types of configuration of four points on $\mathbb{C P}^{1}$ together with the zero polynomial. These types are denoted (I, II, III, D, N, 0), following the well-known application of this classification to Weyl tensors. It is easy to see that the gauge field equation on the trivial (0) Riccati space is the Nahm equation. One can also observe that the gauge field equations on the nontrivial Riccati spaces are equivalent to (strong) isomonodromy equations for a connection with four poles on $\mathbb{C P}^{1}$, the configuration of the poles corresponding to the type of Riccati space. This leads to a uniform Lax pair for these problems, which reduces to the usual Lax pair for the Nahm equation in the trivial case.

The theory of this paper would be very dry without examples and applications, so I intersperse the main development with interludes, which motivate or illustrate the theory, yet are, to varying degrees, self-contained. The first interlude, Section 3, relates the approach of Section 2 to other studies of selfdual Bianchi metrics [14, 15, 47, 53, 54, 55].

The second interlude, Section 5, provides a simple a priori explanation for the conformal invariance of Hitchin's selfduality equation on a Riemann surface [30] (and of course, reductions 
from Kleinian signature also give conformally invariant equations: harmonic maps into a Lie group, and the principal chiral model). This demystifies this conformal invariance: it is, after all, a consequence of conformal invariance in four dimensions.

The third interlude, Section 7, is a unified treatment of various constructions (or at least interpretations) of hypercomplex and hyperkähler structures. My aim here is three-fold:

(i) to provide geometrical descriptions of the well-known Mason-Newman [40], AshtekarJacobson-Smolin [2], and Park-Ward [46, 62] constructions of hyperkähler metrics from lower-dimensional gauge fields;

(ii) to give, at the same time, hypercomplex generalizations, following [17, 26, 33, 37];

(iii) to prove that all hypercomplex and hyperkähler structures are locally obtained from any of these constructions, in a way that is manifestly compatible with any reality conditions, and that clarifies the extra choice that needs to be made to reduce a hypercomplex or hyperkähler structure to a solution of the relevant gauge field equation.

I hope that the overview provided here is useful, at least to the reader who is not familiar with the treatments in the physics literature. In addition this work answers - and extends to the hypercomplex case - a question of Ward [62], who conjectured that any hyperkähler metric could be obtained from Hitchin's selfduality equation, with gauge group $\operatorname{SDiff}\left(\Sigma^{2}\right)$. I also show how this description gives a Euclidean analogue of Plebanski's heavenly equations, which is well-adapted to the study of hyperkähler metrics on elliptic fibrations.

The fourth interlude, Section 9, is a two-part analysis of hyperCR Einstein-Weyl spaces, the three-dimensional analogue of hypercomplex structures. In the first part, following an approach of Tod [56], the hyperCR Einstein-Weyl equation is shown to be equivalent to the $\operatorname{Diff}\left(S^{1}\right)$ Hitchin equation (revealing a hidden $\mathrm{SO}(3)$ symmetry in the latter). This can be viewed as a three-dimensional version of the constructions of Section 7 although it is remarkable that the fibres are only one-dimensional, since such constructions are not sufficiently general in four dimensions. In the second part, Einstein-Weyl spaces admitting a dimensional reduction with geodesic one-dimensional fibres are studied. They are shown to be hyperCR, and the quotient spaces are trivial or spherical spinor-vortex geometries. Conversely, any shear-free geodesic congruence on a hyperCR Einstein-Weyl space defines a generalized dimensional reduction.

The final interlude again consists of two-parts. The first shows that hyperCR Einstein-Weyl spaces may also be constructed from the $\operatorname{Diff}\left(\Sigma^{2}\right)$ Nahm equations. In the second part, I present a proof that the well-known $\mathrm{SU}(\infty)$ Toda field equation $u_{x x}+u_{y y}+\left(e^{u}\right)_{z z}=0$ and the $\mathrm{dKP}$ equation $u_{y y}=\left(u_{t}-u u_{x}\right)_{x}$ are both equivalent to generalized Nahm equations, via a hodograph transformation (cf. [20] in the dKP case). Even these results are to a large extent self-contained, although the the work of Section 8 shows that the construction is a special case of the general theory, while Section 10 shows that the backgrounds for these generalized Nahm equations are the type (D) and (N) Riccati spaces respectively. To the best of my knowledge, all examples of hodograph solutions to the $\mathrm{SU}(\infty)$ Toda field equation or dKP equation arise in this way from explicit solutions of a generalized Nahm equation. I end the interlude by discussing these examples.

\section{Addenda}

The majority of this paper was written in the period 1999-2001, and the present content is not substantially different from a January 2002 version which has been posted on my academic home page since that time. The intervening 12 years have seen many advances in the field (for instance, by Dunajski and his collaborators [67, 68, 69, 70, 71]), and I have collected a few of the most closely related papers as "Additional references" at the end of the bibliography. 
Some of these works develop ideas from the 2002 version of this paper, or discover related ideas independently.

There has also been much work on null reductions and geometries, which were only touched upon in the original version of this paper. A major driving force has been the introduction of a global twistor theory of holomorphic discs by LeBrun and Mason [77, 78, 80, 82], both for Kleinian signature selfdual conformal structures and 2-dimensional projective structures. Dunajski and West [72, 73] established a relationship between these structures by considering selfdual conformal structures with a null conformal Killing vector field. As explained in [65], the natural context for their construction is the null reduction of selfdual conformal structures along a $\beta$-surface foliation. The quotient is a surface with a natural projective structure structure, and the gauge field equations are projectively invariant on this background geometry. In [81] the global Mason-LeBrun theories are related by this construction. More recently, similar methods have been applied in Einstein-Weyl geometry [79, 83].

One of the most intriguing recent developments has been the introduction, by Ferapontov and his coworkers, of the method of hydrodynamic reductions to analyse integrability. This was applied in [74] to study the central quadric Ansatz and its relation to the Painlevé equations. In doing so, they independently rediscovered equations equivalent to the $\operatorname{SDiff}\left(\Sigma^{2}\right)$ generalized Nahm equations on Riccati spaces. More recently, in [75], Ferapontov and Kruglikov construct a Weyl structure from the formal linearization of a second-order PDE in three dimensions, and show that it is Einstein-Weyl for all solutions if and only if the system is integrable. This and related conjectures for second-order PDEs in four dimensions suggest deep connections with the integrable background geometry concept.

For these reasons, the main (odd-numbered) sections have been updated with addenda which place the above works in the framework of this paper. Section 12 and Subsection 11.2 have also been updated to reflect the exciting new directions that are currently unfolding.

\section{Notation}

In order to manipulate, in a tensorial way, the objects and structures entering into the equations and constructions of this paper, it will be convenient to employ the formalism of densities. If $V$ is a real $n$-dimensional vector space and $w$ any real number, then the oriented one-dimensional linear space $L^{w}=L_{V}^{w}$ carrying the representation $A \mapsto|\operatorname{det} A|^{w / n}$ of $\mathrm{GL}(V)$ is called the space of densities of weight $w$ or $w$-densities. It can be constructed canonically as the space of maps $\rho:\left(\wedge^{n} V\right) \backslash 0 \rightarrow \mathbb{R}$ such that $\rho(\lambda \omega)=|\lambda|^{-w / n} \rho(\omega)$ for all $\lambda \in \mathbb{R}^{\times}$and $\omega \in\left(\wedge^{n} V\right) \backslash 0$.

For a vector bundle $\mathcal{V} \rightarrow M$ this construction yields an oriented real line bundle $L_{\mathcal{V}}^{w}$, a density line bundle. If $\mathcal{V}$ is oriented and of rank $n$, then $L_{\mathcal{V}}^{-n}$ is canonically isomorphic to $\wedge^{n} \mathcal{V}$; indeed an orientation may be defined as an orientation form $* 1 \in \mathrm{C}^{\infty}\left(M, L_{\mathcal{V}}^{n} \wedge^{n} \mathcal{V}^{*}\right)$. (Here and elsewhere, when tensoring with a density line bundle, I shall often omit the tensor product sign.) More generally, the Hodge star operator is the isomorphism

$$
*: L_{\mathcal{V}}^{w-k} \wedge^{k} \mathcal{V} \rightarrow L_{\mathcal{V}}^{w+n-k} \wedge^{n-k} \mathcal{V}^{*}
$$

determined by the nondegenerate pairing $\wedge^{k} \mathcal{V}^{*} \otimes \wedge^{n-k} \mathcal{V}^{*} \rightarrow \wedge^{n} \mathcal{V}^{*} \cong L_{\mathcal{V}}^{-n}$.

A conformal structure on $\mathcal{V}$ is a nondegenerate symmetric bilinear form on $\mathcal{V}$ with values in $L_{\mathcal{V}}^{2}$, or equivalently a metric on $L_{\mathcal{V}}^{-1} \mathcal{V}$. The conformal inner product of sections $X, Y$ is $\langle X, Y\rangle \in \mathrm{C}^{\infty}\left(M, L_{\mathcal{V}}^{2}\right)$ and the conformal structure itself may be viewed as a section $\mathrm{c} \in$ $\mathrm{C}^{\infty}\left(M, L_{\mathcal{V}}^{2} S^{2} \mathcal{V}^{*}\right)$. I shall make free use of the isomorphism between $L_{\mathcal{V}}^{w-k} \wedge^{k} \mathcal{V}$ and $L_{\mathcal{V}}^{w+k} \wedge^{k} \mathcal{V}^{*}$ given by a conformal structure.

When $\mathcal{V}$ is the tangent bundle of $M, L_{M}^{w}=L_{T M}^{w}$ is called the bundle of $w$-densities of $M$, denoted $L^{w}$ when $M$ is understood. The line bundles $L^{w}$ are trivializable and a nonvanishing 
(usually positive) section $\mu$ of $L=L^{1}$ will be called a length scale or gauge. I shall also say that tensors in $L^{w} \otimes(T M)^{j} \otimes\left(T^{*} M\right)^{k}$ have weight $w+j-k$.

A Weyl derivative is a covariant derivative $D$ on $L$. It induces covariant derivatives on $L^{w}$ for all $w$. If $M$ is conformal, i.e., there is a conformal structure c on $T M$, then any Weyl derivative induces (via the Koszul formula) a Weyl connection: a torsion-free connection $D$ on $T M$ with $D \mathrm{c}=0$. Compatible Riemannian metrics $g$ correspond to length scales $\mu$, and the Weyl connection induced by the Weyl derivative preserving $\mu$ is, of course, the Levi-Civita connection of $g=\mu^{-2} \mathrm{c}$.

The curvature $R^{D}$ of a Weyl connection $D$, as a $\mathfrak{c o}(T M)$-valued 2-form, decomposes as

$$
R_{X, Y}^{D}=W_{X, Y}-\llbracket r^{D}(X), Y \rrbracket+\llbracket r^{D}(Y), X \rrbracket .
$$

Here $W$ is the Weyl curvature of the conformal structure, an so(TM)-valued 2-form independent of the choice of $D$, and $r^{D}$ is a covector valued 1-form, the (normalized) Ricci curvature of $D$. For a 1-form $\gamma$ and tangent vector $X, \llbracket \gamma, X \rrbracket=\gamma(X)$ id $+\gamma \Delta X$, where $(\gamma \Delta X)(Y)=\gamma(Y) X-$ $\langle X, Y\rangle \gamma$. This bracket is part of a Lie algebra structure on $T M \oplus \mathfrak{c o}(T M) \oplus T^{*} M$ and the same notation will be used for the commutator bracket in $\mathfrak{c o}(T M)$.

The normalized Ricci curvature decomposes into a symmetric traceless part $r_{0}^{D}$, a scalar part $\mathrm{scal}^{D}$ (the scalar curvature) and a skew part, which is just a multiple of the curvature of $D$ on $L$ called the Faraday curvature $F^{D}$. In practice a Weyl derivative is described by its connection 1 -form $\omega$ relative to a length scale: $\omega$ is called the Weyl 1 -form, and $F^{D}=\mathrm{d} \omega$. If $F^{D}=0$ then $D$ is said to be closed. It follows that there are local length scales $\mu$ with $D \mu=0$. If such a length scale exists globally then $D$ is said to be exact.

The above constructions can also be carried out locally on complex manifolds, except that $L^{1}$ is now a choice of local $n$th root of $\wedge^{n} T M$.

Although confusion with the index $i$ is unlikely, as a courtesy to the reader, I denote the (chosen) square root of -1 by $\boldsymbol{i}$.

\section{Twistors and Lax pairs}

Several of the results in this paper were motivated by twistor or integrable systems methods: in particular the idea of generalized dimensional reduction arises naturally when one considers holomorphic foliations of twistor spaces. However, I have deliberately suppressed discussion of twistor spaces and Lax pairs, for at least two reasons: first, to make the paper accessible to the reader not familiar with these ideas; second, because I believe it is a useful to present all calculations and formulae in purely differential geometric terms - it is often impossible to carry out twistor constructions in practice. An unfortunate consequence is that some of the results and formulae appear miraculous: the twistor point of view provides a quick way to see why such results are true, while the Lax pair formalism provides one way to carry out more detailed calculations.

\section{Selfdual spaces and the background geometries}

On a conformal 4-manifold, the Hodge star operator is an involution on 2-forms, so there is a decomposition $\wedge^{2} T^{*} M=\wedge_{+}^{2} T^{*} M \oplus \wedge_{-}^{2} T^{*} M$, and the eigenspaces $\wedge_{ \pm}^{2} T^{*} M$ are called the selfdual and antiselfdual 2-forms. This induces a similar decomposition $\operatorname{so}(T M)=\mathrm{so}_{+}(T M) \oplus$ so- $(T M)$ of the skew endomorphisms of $T M$. The Weyl curvature $W$ splits as a sum of selfdual and antiselfdual 2-forms $W^{ \pm}$with values in $\operatorname{so}(T M)$ : in fact $W^{+}$is $\mathrm{so}_{+}(T M)$-valued and $W^{-}$is so- $(T M)$-valued (essentially because $W$ is traceless).

A selfdual space is a conformal 4-manifold with selfdual Weyl curvature, i.e., $W^{-}=0$. 
In [37], Joyce studied selfdual spaces with a surface-orthogonal action of the torus $T^{2}$ by conformal transformations, and found new explicit selfdual conformal metrics on connected sums of complex projective planes. The key idea in his approach is the use of conformal connections with torsion, and the following observation.

Lemma 2.1 (see Joyce [37]). Let $(M, \mathrm{c})$ be an oriented conformal 4-manifold and $\mathcal{D}$ a conformal connection (i.e., $\mathcal{D} \mathrm{c}=0$, but $\mathcal{D}$ may have torsion). Suppose that the antiselfdual part of the torsion of $\mathcal{D}$ is tracelike. Then $(M, \mathrm{c})$ is selfdual if and only if the Weyl part of the curvature of $\mathcal{D}$ is selfdual.

When the torsion is selfdual and traceless, this lemma follows easily, since $\mathcal{D}$ then differs from a Weyl connection by an $\mathrm{so}_{+}(T M)$-valued 1-form, so that the so- $(T M)$-valued part of the curvature of $\mathcal{D}$ agrees with that of the Weyl connection. The general case is a consequence of the fact that the trace parts of the torsion (which are 1-forms) cannot contribute to the Weyl part of the curvature.

I shall refer to this result as Joyce's lemma: although simple, and perhaps previously known, its application by Joyce [37] was one of the main motivations for the present work. Indeed, for a conformal manifold with a surface-orthogonal $T^{2}$-action, Joyce constructed, on the open set where the torus acts freely, a conformal connection with torsion, and hence separated the selfduality equation for the conformal structure into a nonlinear equation for a quotient geometry and a linear equation defined on this background. He then showed that the quotient geometry in this case is the hyperbolic plane, and superposed known solutions of the linear equation to find new explicit metrics.

In this section, Joyce's lemma will be applied to selfdual spaces with any freely acting group of conformal transformations, and a large class of dimensional reductions of the selfduality equation will be obtained. The restriction to freely acting groups of symmetries will also be relaxed later, leading to a generalized version of dimensional reduction.

Let $M$ be a conformal manifold with a free proper action of a group $H$, so that $M$ is a principal $H$-bundle over the orbit space $Q=M / H$. The generators of the action form a Lie algebra $\mathfrak{h}$ of vector fields on $M$ and pointwise evaluation defines an isomorphism $M \times \mathfrak{h} \rightarrow V M$, where $V M$ is the vertical bundle of $M \rightarrow Q$. Now suppose that $H$ acts conformally with nondegenerate orbits. Then $Q$ is a conformal manifold and the horizontal distribution $V M^{\perp} \leqslant T M$ defines a principal $H$-connection $\alpha$ on $\pi: M \rightarrow Q$; furthermore, $V M$ is isomorphic to the pullback of a conformal vector bundle $\mathcal{V} \rightarrow Q$ with $L_{\mathcal{V}}^{1}=L_{Q}^{1}$. Although $M \times \mathfrak{h}$ and $\pi^{*} \mathcal{V}$ are both isomorphic to $V M$, it will be crucial in the following to distinguish between them, since the trivialization of $V M$ given by the $H$-action is not, in general, compatible with the conformal structure. However, the isomorphism $\pi^{*} \mathcal{V} \rightarrow M \times \mathfrak{h}$ is $H$-equivariant, so it may be viewed as a bundle isomorphism $\varphi: \mathcal{V} \rightarrow \mathfrak{h}_{Q}$ over $Q$, where $\mathfrak{h}_{Q}=M \times_{H} \mathfrak{h}$. To summarize, the conformal geometry of $M$ is encoded by:

- a conformal structure on $Q$;

- a conformal vector bundle $\mathcal{V} \rightarrow Q$ with $L_{\mathcal{V}}^{1}=L_{Q}^{1}$;

- a principal $H$-connection $\alpha$ on $M \rightarrow Q$;

- a bundle isomorphism $\varphi: \mathcal{V} \rightarrow \mathfrak{h}_{Q}$ over $Q$.

The data $(\alpha, \varphi)$ identify the tangent bundle $T M$ with the pullback of $\mathcal{V} \oplus T Q$ : sections of $\mathcal{V}$ or $T Q$ will be denoted $U, V, W$ or $X, Y, Z$ respectively, and identified with invariant vector fields on $M$.

The curvature of the principal connection $\alpha$ on $\pi: M \rightarrow Q$ is an $\mathfrak{h}_{Q^{-}}$valued 2 -form $F^{\alpha}$, given by minus the Frobenius curvature of the horizontal distribution: $F^{\alpha}(X, Y)=-\varphi([X, Y])$, where $\varphi$ extended by zero from $\mathcal{V}$ to $\mathcal{V} \oplus T Q$. 
Choose a conformal connection $D$ on $\mathcal{V}$ over $Q$. This induces a Weyl derivative on $L_{\mathcal{V}}^{1}=L_{Q}^{1}$, hence a torsion-free conformal connection on $T Q$ and a direct sum connection on $\mathcal{V} \oplus T Q$. These conformal connections will be denoted by $D$, as will the pullback connection on $T M=$ $\pi^{*}(\mathcal{V} \oplus T Q)$, which is conformal, but not torsion-free in general: the fibres of $\pi: M \rightarrow Q$ need not be umbilic unless $Q$ is three-dimensional, the nonlinear connection on $M \rightarrow Q$ need not be flat unless $Q$ is one-dimensional, and the sections of $\mathcal{V}$ parallel along the fibres of $\pi$ will have nontrivial Lie brackets unless $\mathfrak{h}$ is Abelian.

In order to apply Joyce's lemma, the torsion needs to be reduced. To do this, introduce $\psi: \wedge^{2} T Q \rightarrow \mathcal{V}$ and $\mathcal{C}: S_{0}^{2} \mathcal{V} \rightarrow T Q$, and define $\mathcal{D}=D+\widehat{\mathcal{C}}+\widehat{\psi}$, where $\widehat{\mathcal{C}}$ and $\widehat{\psi}$ are the sections of $T^{*} M \otimes \operatorname{so}(T M)$ given by

$$
\begin{aligned}
& \widehat{\mathcal{C}}_{U+X}(V+Y)=\mathcal{C}(U, V)-\langle\mathcal{C}(U, \cdot), Y\rangle, \\
& 2 \widehat{\psi}_{U+X}(V+Y)=\langle\psi(X, \cdot), V\rangle+\langle\psi(Y, \cdot), U\rangle-\psi(X, Y) .
\end{aligned}
$$

The idea is that $\psi$ will compensate for the curvature of the horizontal distribution, while $\mathcal{C}$ will offset the traceless second fundamental form of the fibres. It will then be possible to make the torsion selfdual by the choice of $D$.

The torsion $T^{\mathcal{D}}$ of this modified conformal connection may be computed by applying it to invariant vector fields, as long as one is careful that the Lie bracket on invariant vertical vector fields is minus the Lie bracket on the Lie algebra $\mathfrak{h}$ of generators of the action, hence minus the bracket on the associated Lie algebra bundle $\mathfrak{h}_{Q}$ over $Q$

$$
\begin{aligned}
& T^{\mathcal{D}}(U, V)=\varphi^{-1}[\varphi(U), \varphi(V)]_{\mathfrak{h}}, \\
& T^{\mathcal{D}}(U, X)=\varphi^{-1} D_{X}^{\alpha} \varphi(U)-\langle\mathcal{C}(U), X\rangle, \\
& T^{\mathcal{D}}(X, Y)=\varphi^{-1} F^{\alpha}(X, Y)-\psi(X, Y) .
\end{aligned}
$$

Here $D^{\alpha}$ denotes the connection on $\pi^{*}\left(\mathcal{V} \otimes \mathfrak{h}_{Q}\right)$ induced by $D$ and $\alpha$. Note that the torsion is vertical-valued.

The computation of the curvature is a little more complicated. First of all the curvatures of $\mathcal{D}$ and $D($ on $T M$ ) are related by

$$
R_{U+X, V+Y}^{\mathcal{D}}=R_{U+X, V+Y}^{D}+\mathrm{d}^{D}(\widehat{\mathcal{C}}+\widehat{\psi})_{U+X, V+Y}+\llbracket(\widehat{\mathcal{C}}+\widehat{\psi})_{U+X},(\widehat{\mathcal{C}}+\widehat{\psi})_{V+Y} \rrbracket .
$$

The second term is the twisted exterior derivative of so(TM)-valued 1-forms and must be handled carefully, since $D$ has torsion. Relating this to the torsion of $\mathcal{D}$ gives

$$
\begin{aligned}
\mathrm{d}^{D}(\widehat{\mathcal{C}}+\widehat{\psi})_{U+X, V+Y}= & D_{U+X}(\widehat{\mathcal{C}}+\widehat{\psi})_{V+Y}-D_{V+Y}(\widehat{\mathcal{C}}+\widehat{\psi})_{U+X} \\
& +(\widehat{\mathcal{C}}+\widehat{\psi})_{T^{\mathcal{D}}(U+X, V+Y)}+(\widehat{\mathcal{C}}+\widehat{\psi})_{\psi(X, Y)}+(\widehat{\mathcal{C}}+\widehat{\psi})_{\langle\mathcal{C}(U), Y\rangle-\langle\mathcal{C}(V), X\rangle}
\end{aligned}
$$

Notice that the derivatives in vertical directions are zero, since the connection is a pullback connection and $\widehat{\mathcal{C}}, \widehat{\psi}$ are invariant. Also, $R^{D}$ is horizontal. Hence putting everything together leads to the following formula for the "torsion-adjusted" curvature of $\mathcal{D}$

$$
\begin{aligned}
R_{U+X, V+Y}^{\mathcal{D}, t a}:= & R_{U+X, V+Y}^{\mathcal{D}}-(\widehat{\mathcal{C}}+\widehat{\psi})_{T}^{\mathcal{D}}(U+X, V+Y) \\
= & R_{X, Y}^{D}+D_{X} \widehat{\mathcal{C}}_{V}-D_{Y} \widehat{\mathcal{C}}_{U}+D_{X} \widehat{\psi}_{V}-D_{Y} \widehat{\psi}_{U} \\
& +\widehat{\mathcal{C}}_{\psi(X, Y)}+\widehat{\psi}_{\langle\mathcal{C}(U), Y\rangle}-\widehat{\psi}_{\langle\mathcal{C}(V), X\rangle}+\llbracket \widehat{\psi}_{U+X}, \widehat{\mathcal{C}}_{V} \rrbracket+\llbracket \widehat{\mathcal{C}}_{U}, \widehat{\psi}_{V+Y} \rrbracket \\
& +\widehat{\mathcal{C}}_{\langle\mathcal{C}(U), Y\rangle}-\widehat{\mathcal{C}}_{\langle\mathcal{C}(V), X\rangle}+\llbracket \widehat{\mathcal{C}}_{U}, \widehat{\mathcal{C}}_{V} \rrbracket+\widehat{\psi}_{\psi(X, Y)}+\llbracket \widehat{\psi}_{U+X}, \widehat{\psi}_{V+Y} \rrbracket .
\end{aligned}
$$

Now suppose that $T^{\mathcal{D}}$ is selfdual. Then, by Joyce's lemma, the Weyl curvature $W$ of $M$ is selfdual if and only if the Weyl part of this formula is selfdual. This condition is a nonlinear 
differential equation on $Q$ defining a reduced background geometry. A key feature of the formula is that the right hand side is manifestly independent of $(\alpha, \varphi)$ and so the group structure of $H$ decouples from the reduced background geometry.

The details depend on the dimension; in particular, ensuring that the torsion is selfdual constrains $D$. In the following subsections, I will explain these constraints and obtain the background geometries explicitly. For later use I will also introduce individual notations for the geometries in each dimension. As far as possible, the notation will be chosen to be consistent with existing usage. The case of a four-dimensional group acting on itself is left as an exercise: a field $\mathcal{Y} \in \wedge_{-}^{2} \mathcal{V}^{*} \otimes \mathcal{V}$ is needed here to make the torsion selfdual; see Remark 7.9.

\subsection{Reduction to one dimension}

In this case $Q$ is a oriented curve, with weightless unit tangent $\xi$, and $\psi=0$. Write $\mathcal{C}(U, V)=$ $\langle\mathcal{B}(U), V\rangle \xi$, so that $\mathcal{B}$ is a symmetric traceless endomorphism of weight -1 . I will denote the curve $Q$ by $C$ and the bundle $\mathcal{V}$ by $\mathcal{E}$. Thus $\mathcal{E}$ is a rank 3 conformal vector bundle over $C$ with $L_{\mathcal{E}}^{1}=L_{C}^{1}=T C$, the last identification being given by $\xi$. The Hodge star operator is an isomorphism between $\wedge^{2} \mathcal{E}$ and $T C \otimes \mathcal{E}=L^{1} \mathcal{E}$.

The two components of the torsion of $\mathcal{D}$ are given by

$$
T^{\mathcal{D}}(U, V)=\varphi^{-1}[\varphi(U), \varphi(V)]_{\mathfrak{h}}, \quad T^{\mathcal{D}}(U, \xi)=\varphi^{-1} D_{\xi}^{\alpha} \varphi(U)-\mathcal{B}(U) .
$$

This will be selfdual if and only if

$$
\varphi^{-1}\left(D_{\xi}^{\alpha} \varphi-*[\varphi, \varphi]_{\mathfrak{h}}\right)=\mathcal{B} .
$$

Conformal connections $D$ on $\mathcal{E}$ form an affine space modelled on $T^{*} C \otimes \mathfrak{c o}(\mathcal{E})$. Hence $D$ can be chosen uniquely so that the left hand side is symmetric and traceless and this in turn defines $\mathcal{B}$.

The two components of the torsion-adjusted curvature of $\mathcal{D}$ are

$$
R_{U, V}^{\mathcal{D}, t a}=\llbracket \widehat{\mathcal{B}}_{U}, \widehat{\mathcal{B}}_{V} \rrbracket, \quad R_{U, \xi}^{\mathcal{D}, t a}=-D_{\xi} \widehat{\mathcal{B}}_{U}+\widehat{\mathcal{B}}_{\mathcal{B}(U)} .
$$

A straightforward calculation shows that the Weyl part is selfdual if and only if $D_{\xi} \mathcal{B}=2\left(\mathcal{B}^{2}\right)_{0}$, where the subscript zero denotes the traceless part. Since $D$ is flat, this is really just a Riccati equation for a $3 \times 3$ symmetric traceless matrix. On the other hand, the orientation of $C$ is not needed if $\mathcal{B}$ is viewed as an endomorphism-valued 1 -form on $C$.

Definition 2.2. Suppose that $\mathcal{E}$ is a rank 3 conformal vector bundle over a curve $C$ with $L_{\mathcal{E}}^{1}=L_{C}^{1}$. Equip $\mathcal{E}$ with a conformal connection $D$ and a section $\mathcal{B}$ of $T^{*} C \otimes \operatorname{Sym}_{0} \mathcal{E}$. Then $(C, \mathcal{E})$ is said to be a Riccati space if $(D, \mathcal{B})$ satisfy the equation

$$
D \mathcal{B}=2\left(\mathcal{B}^{2}\right)_{0}
$$

Equivalently, with respect to a conformal trivialization of $\mathcal{E}$, the connection $D$ is given by a section $(a, \Theta)$ of $T^{*} C \otimes \mathfrak{c o}(\mathcal{E})=T^{*} C \oplus\left(T^{*} C \otimes \operatorname{so}(\mathcal{E})\right)$ and

$$
\dot{\mathcal{B}}-a \mathcal{B}+\llbracket \Theta, \mathcal{B} \rrbracket=2\left(\mathcal{B}^{2}\right)_{0}
$$

where the dot denotes differentiation with respect to a compatible coordinate. In particular, using a $D$-parallel trivialization of $\mathcal{E}$ and an affine coordinate $r$ (i.e., $D \mathrm{~d} r=0), \mathcal{B}_{r}=2\left(\mathcal{B}^{2}\right)_{0}$.

Joyce's lemma now gives the following result.

Theorem 2.3. Let $M$ be an oriented conformal manifold with a 3-dimensional Lie algebra $\mathfrak{h}$ of linearly independent conformal vector fields such that the projection $\pi$ onto the space of orbits is a submersion over a curve $C$. Let $\mathcal{E}$ be a rank 3 conformal vector bundle on $C$ such that $\pi^{*} \mathcal{E}$ is the vertical bundle of $M$ (trivialized along the fibres by invariant vector fields) and define $(D, \mathcal{B})$ by equation (2.4) as explained above.

Then $M$ is selfdual if and only if $(C, \mathcal{E})$ is a Riccati space. 


\subsection{Reduction to two dimensions}

The two-dimensional geometry is perhaps the richest. In the Euclidean case, $Q$ is a Riemann surface, which will be denoted $N$, and the conformal vector bundle $\mathcal{V}$ has rank two, and so will be viewed as a complex line bundle $\mathcal{W} \rightarrow N$. It will be convenient to view $\varphi: \mathcal{W} \rightarrow \mathfrak{h}_{N}$ as a complex linear map $\frac{1}{2}(\varphi-\boldsymbol{i} \varphi \circ J): \mathcal{W} \rightarrow \mathfrak{h}_{N} \otimes \mathbb{C}$. The constraint $L_{\mathcal{W}}^{1}=L_{N}^{1}$ means that there is a Hermitian metric on $\mathcal{W}^{*} \otimes_{\mathbb{C}} T N$. On fixing orientations, this may also be interpreted as an identification $\mathcal{W} \otimes_{\mathbb{C}} \overline{\mathcal{W}}=T N \otimes_{\mathbb{C}} \overline{T N}$, or $\wedge^{1,1} \mathcal{W}=\wedge^{1,1} T N$. Here the orientations will be chosen so that the induced almost complex structure $J$ on $T M=\pi^{*}(\mathcal{W} \oplus T N)$ is selfdual, i.e., the corresponding weightless 2 -form $\Omega \in \mathrm{C}^{\infty}\left(M, L^{2} \wedge^{2} T^{*} M\right)$ is selfdual.

The Hodge star operator on $\wedge^{2} T M$ interchanges the vertical and horizontal components using the identification above, and acts on mixed bivectors by $*(U \wedge X)=-J U \wedge J X$. In particular the torsion of the modified connection $\mathcal{D}$ is selfdual if and only if

$$
\begin{aligned}
& \varphi^{-1}\left(F^{\alpha}-[\varphi, \bar{\varphi}]_{\mathfrak{h}}\right)=\psi, \\
& \varphi^{-1}\left(D_{X}^{\alpha} \varphi(U)+D_{J X}^{\alpha} \varphi(J U)\right)=\langle\mathcal{C}(U), X\rangle+\langle\mathcal{C}(J U), J X\rangle
\end{aligned}
$$

for any vector field $X$ and section $U$ of $\mathcal{W}$. The first equation defines $\psi$ uniquely. For the second equation, note that conformal connections $D$ on $\mathcal{W}$ form an affine space modelled on $T^{*} N \otimes_{\mathbb{R}} \mathfrak{c o}(\mathcal{W})$, and therefore $D$ can be chosen uniquely such that $\varphi^{-1} D_{X}^{\alpha} \varphi$ is symmetric and traceless for all $X$. The remaining ambiguity in $\mathcal{C}$ is fixed by supposing it is complex linear, i.e., a section of $S_{0}^{2} \mathcal{W}^{*} \otimes_{\mathbb{C}} T N$, so that the second equation determines it uniquely. Note though, that the second equation does not use all of $D$ : only the induced holomorphic structure of $\mathcal{W}$ is needed.

For the selfduality of the Weyl curvature, it now suffices to compute $R_{U, Y}^{\mathcal{D}, t a}$ and one additional component. To see this, note first that $\widehat{\psi}$ and $\widehat{\mathcal{C}}$ are so $(T M)$-valued, and therefore no information is lost by considering $R^{\mathcal{D}}$ and $R^{D}$ to be the curvatures on $L^{-1} T M$ rather than $T M$, so that the curvature equation is an identity between so(TM)-valued 2-forms. The selfduality condition is obtained by requiring that the Weyl part of $R^{\mathcal{D}, t a}$ is selfdual. This amounts to considering the traceless part of the induced bundle map from $\wedge_{-}^{2} T M$ to so- $(T M)$. Let $\bar{\Omega}$ and $\bar{J}$ be the sections of these bundles obtained by reversing the orientation of $\Omega$ and $J$ on $T N$. There are therefore three equations to find:

(i) The traceless part of the $\bar{\Omega}^{\perp} \otimes \bar{J}^{\perp}$ component of $R^{\mathcal{D}, t a}$ should be zero.

(ii) The part of $R^{\mathcal{D}, t a}$ in $\bar{\Omega}^{\perp} \otimes\langle\bar{J}\rangle$ should be zero.

(iii) The multiple of $\bar{\Omega} \otimes \bar{J}$ should equal half the trace in $\bar{\Omega}^{\perp} \otimes \bar{J}^{\perp}$.

All of these except (iii) involve considering only the part of $R^{\mathcal{D}, t a}$ in $\bar{\Omega}^{\perp} \otimes \operatorname{so}(T M)$, which involves evaluating $R^{\mathcal{D}, t a}$ on bivector fields of the form $U \wedge Y-*(U \wedge Y)=U \wedge Y+J U \wedge J Y$. An easy calculation gives

$$
\begin{aligned}
R_{U, Y}^{\mathcal{D}, t a}= & -D_{Y} \mathcal{C}(U, \cdot)+D_{Y} \mathcal{C}(U, \cdot)^{\mathrm{T}}-\frac{1}{4} D_{Y} \psi(J U)(J-\bar{J}) \\
& +\mathcal{C}(\langle\mathcal{C}(U), Y\rangle, \cdot)-\mathcal{C}(\langle\mathcal{C}(U), Y\rangle, \cdot)^{\mathrm{T}}-\frac{1}{4}\langle\mathcal{C}(U, J \psi), Y\rangle(3 J-\bar{J})-\frac{1}{4} J \psi(U) J \psi \triangle Y,
\end{aligned}
$$

where ${ }^{\mathrm{T}}$ denotes the transpose, and I have contracted horizontal and vertical skew endomorphisms using $X \triangle Y=\frac{1}{2} \Omega(X, Y)(J-\bar{J})$ and $U \Delta V=\frac{1}{2} \Omega(U, V)(J+\bar{J})$. Also, using $\Omega, \psi$ may be viewed as a section of $\mathcal{W}^{*}$, so that $\psi(X, Y)=2 \Omega(X, Y) J \psi$.

One readily obtains conditions (i) and (ii):

$$
\begin{aligned}
& D_{X} \mathcal{C}(U, \cdot)+D_{J X} \mathcal{C}(J U, \cdot)=0, \\
& \frac{1}{2}\left(D_{X} \psi(U)+D_{J X} \psi(J U)\right)=-3\langle\mathcal{C}(\psi, U), X\rangle,
\end{aligned}
$$

for any vector field $X$ and section $U$ of $\mathcal{W}$. 
It remains to compute condition (iii). For this note that $R^{D}$ is given by $\frac{1}{4} s_{N}(\Omega-\bar{\Omega}) \otimes(J-$ $\bar{J})+\frac{1}{4} s_{\mathcal{W}}(\Omega-\bar{\Omega}) \otimes(J+\bar{J})$, where $s_{N}=\frac{1}{2} \operatorname{scal}_{N}$ and $s_{\mathcal{W}}=\frac{1}{2} \operatorname{scal}_{\mathcal{W}}$ are the normalized scalar curvatures of $D$ on $T N$ and $\mathcal{W}$. This yields, finally,

$$
s_{N}-s_{\mathcal{W}}=|\psi|^{2}-2|\mathcal{C}|^{2} .
$$

These equations, and the equations defining $\mathcal{C}$ and $\psi$, depend only on $D$ through the induced holomorphic structure $\bar{\partial}^{a}$ on $\mathcal{W}$. The conformal connections on $\mathcal{W}$ and $T N$ may be viewed as Chern connections determined by the holomorphic structures on these bundles together with the choice of a Weyl derivative on $L^{1}$. The difference $s_{N}-s_{\mathcal{W}}$ does not depend on the choice of Weyl derivative, since it is the normalized scalar curvature of the Chern connection on the Hermitian line bundle $\mathcal{W}^{-1} T N$.

Aside 2.4. The construction of a "Chern-Weyl" connection does not seem to be known and relates to a simple coordinate-free description (also not well-known) of the usual Chern connection, so I will sketch it here. Let $E$ be a complex vector bundle (over a complex manifold $M, J$ ) with holomorphic structure $\bar{\partial}^{E}$ and compatible conformal metric c: $S_{\mathbb{R}}^{2} E \rightarrow L_{E}^{2}$ with respect to which the complex structure on $E$ is orthogonal; the latter is equivalently a conformal Hermitian structure $\bar{E} \otimes_{\mathbb{C}} E \rightarrow L_{E}^{2} \otimes \mathbb{C}$. (Note that a complex line bundle automatically has a conformal Hermitian structure.) Now given any covariant derivative $D$ on $L_{E}^{1}$, let $\bar{\partial}_{X}^{D}=\frac{1}{2}\left(D_{X}+i D_{J X}\right)$ (for all vector fields $X$ ) be the induced almost holomorphic structure on $L_{E}^{2} \otimes \mathbb{C}$. Then there is a unique conformal Hermitian connection $D^{E}$ on $E$ inducing $D$ on $L_{E}^{1}$. It is given by the formula

$$
\left\langle D_{X}^{E} s_{1}, s_{2}\right\rangle=\bar{\partial}_{X}^{D}\left\langle s_{1}, s_{2}\right\rangle-\left\langle s_{1}, \bar{\partial}_{X}^{E} s_{2}\right\rangle+\left\langle\bar{\partial}_{X}^{E} s_{1}, s_{2}\right\rangle,
$$

where $s_{1}$ and $s_{2}$ are sections of $E$. The proof is immediate (the idea behind the formula is simply that $D^{E}=\partial^{E}+\bar{\partial}^{E}$, where $\partial^{E}$ is the complex-linear part of the derivative). In the case that the covariant derivative on $L_{E}^{1}$ is just a trivialization, so that $\bar{\partial}^{D}=\bar{\partial}$, this is the usual Chern connection by uniqueness.

Definition 2.5. Suppose that $\mathcal{W}$ is a complex line bundle over a Riemann surface $N$ such that $L_{\mathcal{W}}^{1}=L_{N}^{1}$ (i.e., there is a Hermitian metric on $\mathcal{W}^{-1} T N$ ). Equip $\mathcal{W}$ with a holomorphic structure $\bar{\partial}^{a}$, a section $\mathcal{C}$ of $\mathcal{W}^{-2} T N$, and a section $\psi$ of $\mathcal{W}^{-1}$. Then $(N, \mathcal{W})$ is said to be a spinor-vortex space if $\left(\bar{\partial}^{a}, \mathcal{C}, \psi\right)$ satisfy the equations

$$
\begin{aligned}
& \bar{\partial}^{a} \mathcal{C}=0, \\
& \bar{\partial}^{a} \psi=-3 \mathcal{C} \bar{\psi}, \\
& s_{\mathcal{W}^{-1} T N}=\psi \bar{\psi}-2 \mathcal{C} \overline{\mathcal{C}},
\end{aligned}
$$

where $s_{\mathcal{W}^{-1} T N}$ is the normalized scalar curvature of the Chern connection on $\mathcal{W}^{-1} T N$.

Joyce's lemma now gives the following result.

Theorem 2.6. Let $M$ be an oriented conformal manifold with a 2-dimensional Lie algebra $\mathfrak{h}$ of linearly independent conformal vector fields such that the projection $\pi$ onto the space of orbits is a submersion over a Riemann surface $N$. Let $\mathcal{W}$ be a complex line bundle on $N$ such that $\pi^{*} \mathcal{W}$ is the vertical bundle of $M$ (trivialized along the fibres by invariant vector fields) and define $\left(\bar{\partial}^{a}, \mathcal{C}, \psi\right)$ by $(2.5)$ as explained above.

Then $M$ is selfdual if and only if $(N, \mathcal{E})$ is a spinor-vortex space.

Of course there are only two possible 2 -dimensional Lie algebras. In the case that $\mathfrak{h}$ is Abelian and $\psi=0$, this result reduces to the original application of Lemma 2.1 by Joyce [37]. 
Remark 2.7. It is straightforward to adapt the calculations of this subsection to other signatures: in general $N$ is a conformal surface with two line bundles $\mathcal{W}, \widetilde{\mathcal{W}}$ such that $\mathcal{W} \widetilde{\mathcal{W}} \cong$ $T^{1,0} N T^{0,1} N$ where $T^{1,0} N$ and $T^{0,1} N$ are the null line subbundles of $T N \otimes \mathbb{C}$. The line bundles $\mathcal{W}$ and $\widetilde{\mathcal{W}}$ are equipped with "(anti)holomorphic structures" $\tilde{\partial}^{a}, \partial^{a}$ and there are sections $(\mathcal{C}, \widetilde{\mathcal{C}}, \psi, \widetilde{\psi})$ satisfying appropriate reality conditions: in the Euclidean case $\widetilde{\mathcal{W}}=\overline{\mathcal{W}}, \widetilde{\mathcal{C}}=\overline{\mathcal{C}}$ and $\widetilde{\psi}=\bar{\psi}$, but there is also a Lorentzian case, when the fields are all real, and a Euclidean reduction from Kleinian signature $(2,2)$ when $\widetilde{\psi}=-\bar{\psi}$.

\subsection{Reduction to three dimensions}

The reduction to three dimensions is the Jones-Tod correspondence [36], which was one of the motivations for this work. The proof using Joyce's lemma is outlined in [37], so I will only recall the main ideas and definitions.

In this case, $Q$ is an oriented 3 -dimensional conformal manifold, which will be denoted $B$, and $\mathcal{V}$ is an oriented line bundle isomorphic to $L^{1}=L_{B}^{1}$. Note that $\mathcal{C}$ automatically vanishes, and write $\psi=* \omega$, where $\omega$ is a 1 -form on $B$. Then the torsion $T^{\mathcal{D}}$ will be selfdual provided that

$$
*(D \varphi-\omega \varphi)=F^{\alpha} .
$$

(The connection $\alpha$ does not act on $\varphi$ since $\mathfrak{h}$ is Abelian.)

This equation determines $(D, \omega)$ up to the gauge freedom $D \mapsto D+\gamma, \omega \mapsto \omega-\gamma$ for any 1-form $\gamma$ on $B$ (note that $(D+\gamma) \varphi=D \varphi-\gamma \varphi$, since $\varphi$ has weight -1 ). In other words, it determines uniquely the Weyl derivative $D+\omega$. This is called the Jones-Tod Weyl structure.

The gauge freedom can be used to set $D \varphi=0$, or to set $\omega=0$. Taking the latter point of view, the Jones-Tod Weyl structure is just $D$, determined by $* D \varphi=F^{\alpha}$.

The curvature of $\mathcal{D}$ is simply the pullback of the curvature of $D$ on $B$. In particular the Weyl part will be selfdual iff it vanishes and this is readily seen to be equivalent to the vanishing of $r_{0}^{D}$ [37].

Definition 2.8. Suppose that $B$ is a conformal 3-manifold and let $D$ be a Weyl connection on $B$. Then $B$ is said to be an Einstein-Weyl space iff $r_{0}^{D}=0$, i.e., the symmetric traceless Ricci tensor of $D$ vanishes.

Theorem 2.9 ([36]). Let $M$ be an oriented conformal 4-manifold and $K$ a nonvanishing conformal vector field such that the projection $\pi$ of $M$ onto the space of trajectories is a submersion over a conformal manifold B. Equip B with the Jones-Tod Weyl structure.

Then $M$ is selfdual if and only if $B$ is Einstein-Weyl.

All three background geometries are themselves defined by geometric gauge theories: conformal local trivializations of the bundle $\mathcal{V}$ over $Q$ are related by gauge transformations. In the three-dimensional case, $\mathcal{V}$ is simply $L^{1}$, so conformal trivializations are length scales, and this is Weyl's original gauge theory [63]. Analogously, in the one and two-dimensional case, $\mathcal{E}$ and $\mathcal{W}$ should be regarded as part of the geometry of the space, not auxiliary bundles, and these background equations, although gauge-theoretic, should not be confused with the gauge field equations on auxiliary $G$-bundles which will be studied in Section 4.

\section{Addenda: null reductions}

The constructions of this section assume nondegeneracy of the conformal structure c on $M$ along the orbits of the symmetry group $H$. In (real) Euclidean signature, this is automatic, but this is not the case when $M$ has Kleinian signature, nor when $M$ is a holomorphic conformal manifold. When the conformal structure degenerates on the orbits, the reduction is said to be 
null. For the generic local considerations of this paper, I assume that the radical (or kernel) $R_{V}:=V M \cap V M^{\perp}$ of the conformal structure along the $H$ orbits has constant rank (as before, $V M$ denotes the tangent bundle to the $H$ orbits). There are thus three possibilities:

(1) $R_{V}$ has rank one;

(2+) $R_{V}=U_{+}$has rank two and is selfdual;

(2-) $R_{V}=U_{-}$has rank two and is antiselfdual.

In case (1), $R_{V}^{\perp}=V M+V M^{\perp}$ has rank three, and $R_{V}^{\perp} / R_{V}$ is the sum of two null subbundles with a nondegenerate pairing between them. Their inverse images $U_{ \pm}$in $R_{V}^{\perp}$ are selfdual and antiselfdual null 2-plane distributions. There are several subcases to consider here, depending on the rank of $V M$. If either $U_{ \pm} \subseteq V M$ then $V M$ must have rank 3, in which case $V M^{\perp} \subset V M$, hence $V M^{\perp}=R_{V}$ and $R_{V}^{\perp}=V M$, so $V M=U_{+}+U_{-}$. If $V M$ has rank two, then $T M=$ $V M+U_{+}+U_{-}$, and clearly if $\operatorname{rank} V_{M}=1$, then $R_{V}=V M$.

Cases (2士) are simpler, at least when $\operatorname{rank} V M=2$, so $V M=R_{V}$ is a bundle of selfdual or antiselfdual 2-planes. I concentrate on these two cases here. One justification for such a focus is that in the other cases, one may be able to prove that the distributions $U_{+}$or $U_{-}$are integrable, and hence study the reduction in terms of their leaf spaces. For instance, Dunajski-West [72] establish such an integrability result when $\operatorname{rank} V M=1$.

Suppose then that $\pi: M \rightarrow Q=M / H$ is a principal $H$-bundle over a manifold $Q$ such that $V M$ has rank two and is totally null. As $V M^{\perp}=V M$, neither $V M$ nor $T Q$ inherit conformal structures from $M$; instead there is a nondegenerate pairing $V M \times \pi^{*} T Q \rightarrow L^{2}$. This pairing in $H$-invariant, and thus identifies $V M=\pi^{*}\left(T^{*} Q \otimes \mathcal{V}\right)$, for a line bundle $\mathcal{V} \rightarrow Q$ (whose pullback to $M$ will be identified with $L^{2}$ ). As in the nondegenerate setting the isomorphism of $V M$ with $M \times \mathfrak{h}$ descends to a bundle isomorphism $\varphi: T^{*} Q \otimes \mathcal{V} \rightarrow \mathfrak{h}_{Q}:=M \times_{H} \mathfrak{h}$ over $Q$. There is no canonical splitting of the short exact sequence $0 \rightarrow \pi^{*} T^{*} Q \otimes \mathcal{V} \rightarrow T M \rightarrow \pi^{*} T Q \rightarrow 0$, but such a splitting may be chosen so that the complementary subbundle to $V M$ is $H$-invariant and null. This yields a principal $H$-connection $\alpha$ on $\pi: M \rightarrow Q$.

A torsion-free connection on $T N$ and a connection on $\mathcal{V}$ together induce a conformal connection $D$ on $T M$ with vertical-valued torsion as before. A conformal connection $\mathcal{D}$ with selfdual torsion may be obtained by adding correction terms to $D$. Compared to the nondegenerate case, the correction term $\mathcal{C}$ may be absorbed into the choice of $D$, and replaced by a vertical correction $\chi: \wedge^{2}\left(T^{*} Q \otimes \mathcal{V}\right) \rightarrow T^{*} Q \otimes \mathcal{V}$. Thus $\mathcal{D}=D+\widehat{\chi}+\widehat{\psi}$, where $\psi: \wedge^{2} T Q \rightarrow T^{*} Q \otimes \mathcal{V}$ as before, and

$$
\begin{aligned}
& 2 \widehat{\chi}_{U+X}(V+Y)=\langle\chi(U, \cdot), Y\rangle+\langle\chi(V, \cdot), X\rangle-\chi(U, V), \\
& 2 \widehat{\psi}_{U+X}(V+Y)=\langle\psi(X, \cdot), Y\rangle+\langle\psi(Y, \cdot), X\rangle-\psi(X, Y) .
\end{aligned}
$$

The torsion satisfies

$$
\begin{aligned}
& T^{\mathcal{D}}(U, V)=\varphi^{-1}[\varphi(U), \varphi(V)]_{\mathfrak{h}}-\chi(U, V), \\
& T^{\mathcal{D}}(U, X)=\varphi^{-1} D_{X}^{\alpha} \varphi(U), \\
& T^{\mathcal{D}}(X, Y)=\varphi^{-1} F^{\alpha}(X, Y)-\psi(X, Y),
\end{aligned}
$$

while the curvature computes to

$$
\begin{aligned}
R_{U+X, V+Y}^{\mathcal{D}}= & R_{U+X, V+Y}^{D}+(\widehat{\chi}+\widehat{\psi})_{T^{\mathcal{D}}(U+X, V+Y)} \\
& +D_{U+X}(\widehat{\chi}+\widehat{\psi})_{V+Y}-D_{V+Y}(\widehat{\chi}+\widehat{\psi})_{U+X} \\
& +(\widehat{\chi}+\widehat{\psi})_{\psi(X, Y)}+(\widehat{\chi}+\widehat{\psi})_{\chi(U, V)}+\llbracket(\widehat{\chi}+\widehat{\psi})_{U+X},(\widehat{\chi}+\widehat{\psi})_{V+Y} \rrbracket .
\end{aligned}
$$


Hence the torsion-adjusted curvature is

$$
\begin{aligned}
R_{U+X, V+Y}^{\mathcal{D}, t a}= & R_{X, Y}^{D}+\llbracket(\widehat{\chi}+\widehat{\psi})_{X},(\widehat{\chi}+\widehat{\psi})_{Y} \rrbracket+\widehat{\chi}_{\psi(X, Y)} \\
& +D_{X}(\widehat{\chi}+\widehat{\psi})_{Y}-D_{Y}(\widehat{\chi}+\widehat{\psi})_{X}+D_{X} \widehat{\chi}_{V}-D_{Y} \widehat{\chi}_{U} \\
& +\llbracket(\widehat{\chi}+\widehat{\psi})_{X}, \widehat{\chi}_{V} \rrbracket+\llbracket \widehat{\chi}_{U},(\widehat{\chi}+\widehat{\psi})_{Y} \rrbracket+\llbracket \widehat{\chi}_{U}, \widehat{\chi}_{V} \rrbracket+\widehat{\chi}_{\chi}(U, V) .
\end{aligned}
$$

\section{Antiselfdual ( $\alpha$-surface) reduction}

When $V M$ is antiselfdual, the fibres of $\pi: M \rightarrow Q$ are $\alpha$-surfaces (i.e., they correspond to points in the twistor space of $M)$. Under the isomorphism of $T M$ with $\pi^{*}\left(T^{*} Q \otimes \mathcal{V} \oplus T Q\right)$, the antiselfdual bivectors decompose into three rank one subbundles: $\wedge^{2}\left(T^{*} Q \otimes \mathcal{V}\right), \wedge^{2} T Q$, and the tracelike part of $T Q \otimes T^{*} Q \otimes \mathcal{V}$. The corresponding decomposition of so_ $(T M)$ has summands $\mathrm{Hom}_{-}\left(T Q, T^{*} Q \otimes \mathcal{V}\right), \mathrm{Hom}_{-}\left(T^{*} Q \otimes \mathcal{V}, T Q\right)$ and the span of $\operatorname{id}_{T^{*} Q \otimes \mathcal{V}}-\mathrm{id}_{T Q}$; here Hom_ denotes the subbundle of skew symmetric operators.

In order to interpret equations (2.10)-(2.12) and (2.13), it is convenient to set $\mathcal{V}=\mathcal{L}^{2} \otimes \wedge^{2} T Q$ for a line bundle $\mathcal{L}$, so that $\varphi, \psi$ and $\chi$ may be viewed as 1 -forms on $Q$, with values in $\mathcal{L}^{2} \otimes \mathfrak{h}_{Q}$, $\mathcal{L}^{-2}$ and $\mathcal{L}^{2}$ respectively.

Then $T^{\mathcal{D}}$ is selfdual iff $\chi \wedge \varphi=\frac{1}{2}[\varphi \wedge \varphi]_{\mathfrak{h}}, \psi \wedge \varphi=F^{\alpha}$ and $\mathrm{d}^{D, \alpha} \varphi=0$. The first two equations determine $\chi$ and $\psi$ uniquely (since $\varphi: T N \rightarrow \mathcal{L} \otimes \mathfrak{h}_{Q}$ is injective), while the third depends only on (and essentially determines) the connection $a$ induced by $D$ on $\mathcal{L}$.

In the expression (2.13) for the torsion-adjusted curvature, very few terms contribute to the antiselfdual Weyl part. For instance, only the trace part of first and last lines contribute, and the latter trace vanishes identically. After some tedious computations, the background equations for the 1 -forms $\psi$ and $\chi$ and the connection $a$ on $\mathcal{L}$ reduce to

$$
\mathrm{d}^{a} \psi=0, \quad \mathrm{~d}^{a} \chi=0, \quad F^{a}=\chi \wedge \psi,
$$

which may be interpreted as the flatness of the connection $\mathrm{d}^{a}+\psi+\chi$ on $\mathcal{L} \oplus \mathcal{L}^{-1}$ (where $\mathrm{d}^{a}$ is the direct sum connection, while $\psi$ and $\chi$ are viewed as 1 -forms with values in $\operatorname{Hom}\left(\mathcal{L}^{-1}, \mathcal{L}\right)$ and $\left.\operatorname{Hom}\left(\mathcal{L}, \mathcal{L}^{-1}\right)\right)$.

\section{Selfdual ( $\beta$-surface) reduction}

In the selfdual case, only the mixed part of $T^{\mathcal{D}}$ has a antiselfdual component, so there is no loss in setting $\psi=\chi=0$. The selfduality of the torsion then reduces to $D^{\alpha} \varphi=\frac{1}{2} \mathrm{~d}^{D^{\alpha}} \varphi$, where $\varphi$ is interpreted as a 1-form on $Q$ as before. This equation determines $D$ up to projective transformation $\left(D_{X} Y \mapsto D_{X} Y+\gamma(X) Y+\gamma(Y) X\right.$ for a 1-form $\left.\gamma\right)$, and the background equations are vacuous. Hence the background geometry is an arbitrary (torsion-free) projective surface $(Q,[D])$; this reduction was obtained in $[72]$ and $[65]$.

\section{Interlude: Bianchi metrics}

Selfdual conformal manifolds with a freely acting three-dimensional symmetry group have been studied in many places $[14,15,31,42,43,47,53,54,55]$. In this interlude, I will show briefly how the direct approach to selfdual Bianchi metrics is related to the Riccati space reduction of the previous section.

For simplicity, I focus on the case of diagonal Bianchi IX metrics. Such a metric may be written in the form

$$
g=w_{1} w_{2} w_{3} \mathrm{~d} t^{2}+\frac{w_{2} w_{3}}{w_{1}} \sigma_{1}^{2}+\frac{w_{3} w_{1}}{w_{2}} \sigma_{2}^{2}+\frac{w_{1} w_{2}}{w_{3}} \sigma_{3}^{2},
$$


where $\sigma_{1}, \sigma_{2}, \sigma_{3}$ are the usual left-invariant 1-forms on $\mathrm{SU}(2)$ and $w_{1}, w_{2}, w_{3}$ are functions of $t$. If $X_{1}, X_{2}, X_{3}$ are the dual vector fields to $\sigma_{1}, \sigma_{2}, \sigma_{3}$, then the vector fields $\partial_{t}, \varphi_{1}=w_{1} X_{1}$, $\varphi_{2}=w_{2} X_{2}$ and $\varphi_{3}=w_{3} X_{3}$ form a conformal frame. Notice that $\dot{\varphi}_{1}-\left[\varphi_{2}, \varphi_{3}\right]$ is the multiple $\dot{w}_{1}-w_{2} w_{3}$ of $X_{1}$, where the dot denotes the derivative with respect to $t$. The other two components are similar. Following [47, 53], write

$$
\begin{aligned}
& \dot{w}_{1}=w_{2} w_{3}-w_{1}\left(A_{2}+A_{3}\right), \\
& \dot{w}_{2}=w_{3} w_{1}-w_{2}\left(A_{3}+A_{1}\right), \\
& \dot{w}_{3}=w_{1} w_{2}-w_{3}\left(A_{1}+A_{2}\right) .
\end{aligned}
$$

Comparing with equation (2.4), observe that the matrix $\mathcal{B}$ is diagonal, with eigenvalues $-\left(A_{2}+\right.$ $\left.A_{3}\right),-\left(A_{3}+A_{1}\right),-\left(A_{1}+A_{2}\right)$. In the approach of the previous section, the conformal gauge freedom is used to set $A_{1}+A_{2}+A_{3}=0$, so that $\mathcal{B}$ is traceless with eigenvalues $A_{1}, A_{2}, A_{3}$. This is not usually done in the literature, because by working with an arbitrary compatible metric, additional (non-conformally-invariant) equations can be imposed. In particular, vacuum metrics - and more generally, Kähler metrics (with antiselfdual complex structure) - are scalarflat. Hence scalar-flatness is often used as a gauge condition, in which case the following well-known system, originating in work of Brioschi, Chazy, Darboux, and Halphen, is obtained:

$$
\begin{aligned}
& \dot{A}_{1}=A_{2} A_{3}-A_{1}\left(A_{2}+A_{3}\right), \\
& \dot{A}_{2}=A_{3} A_{1}-A_{2}\left(A_{3}+A_{1}\right), \\
& \dot{A}_{3}=A_{1} A_{2}-A_{3}\left(A_{1}+A_{2}\right) .
\end{aligned}
$$

Joyce's lemma explains the remarkable fact that this system depends only on $w_{1}, w_{2}, w_{3}$ through the functions $A_{1}, A_{2}, A_{3}$. The trace of this system is the scalar-flat gauge condition: $\dot{A}_{1}+\dot{A}_{2}+$ $\dot{A}_{3}=-A_{2} A_{3}-A_{3} A_{1}-A_{1} A_{2}$, while the traceless part is the selfduality equation for the Weyl curvature - the latter condition is independent of the choice of conformal gauge, and may be rewritten as a matrix Riccati equation in the following way:

$$
\begin{aligned}
& \mathcal{B}=\frac{1}{3}\left[\begin{array}{ccc}
2 A_{1}-A_{2}-A_{3} & 0 & 0 \\
0 & 2 A_{2}-A_{3}-A_{1} & 0 \\
0 & 0 & 2 A_{3}-A_{1}-A_{2}
\end{array}\right], \\
& \dot{\mathcal{B}}-a \mathcal{B}=2\left(\mathcal{B}^{2}\right)_{0},
\end{aligned}
$$

where

$$
a=-\frac{2}{3}\left(A_{1}+A_{2}+A_{3}\right) .
$$

In other words, the matrix Riccati equation is obtained by separating the diagonal matrix $\mathcal{B}+a$ id, with eigenvalues $-\left(A_{2}+A_{3}\right),-\left(A_{3}+A_{1}\right),-\left(A_{1}+A_{2}\right)$, into its trace (which defines the connection $D=\partial_{t}+a$ on the one-dimensional quotient geometry), and its traceless part (which is $\mathcal{B})$.

One advantage of this geometric interpretation is that different gauge conditions can be easily compared. Evidently the condition $a=0$ fixes $t$ up to affine transformations. In order to interpret the scalar-flat gauge condition $\dot{a}=\frac{2}{3}\left(A_{2} A_{3}+A_{3} A_{1}+A_{1} A_{2}\right)$, it is natural and illuminating to express the right hand side in terms of $a$ and $\operatorname{tr} \mathcal{B}^{2}$. The result is

$$
\dot{a}-\frac{1}{2} a^{2}=-\frac{1}{3} \operatorname{tr} \mathcal{B}^{2} .
$$

Note that $\left(\partial_{t}-\frac{1}{2} a\right)\left(\partial_{t}+\frac{1}{2} a\right)=\partial_{t}^{2}+\frac{1}{2} \dot{a}-\frac{1}{4} a^{2}$, so that on sections of $L^{1 / 2}, D^{2}=\partial_{t}^{2}-\frac{1}{6} \operatorname{tr} \mathcal{B}^{2}$. Hence the scalar-flat gauge condition may be interpreted as fixing $t$ to be a projective coordinate with respect to the projective structure $D^{2}+\frac{1}{6} \operatorname{tr} \mathcal{B}^{2}$; this determines $t$ up to a projective transformation. 


\section{The gauge field equations}

Let $\nabla$ be a $G$-connection, on a vector bundle $E$ over a conformal manifold $M$, which is invariant under an action of a group $H$ of conformal transformations. Infinitesimally, the generators form a Lie algebra $\mathfrak{h}$ of conformal vector fields on $M$ and there is an action of these vector fields by Lie derivative on sections of $E$ such that $\mathcal{L}_{\xi} \nabla=0$ for all $\xi \in \mathfrak{h}$. Each $\xi \in \mathfrak{h}$ therefore determines a Higgs field $S_{\xi}$ in the associated Lie algebra bundle $\mathfrak{g}_{M} \leqslant \operatorname{End}(E)$ defined by $S_{\xi} s=\mathcal{L}_{\xi} s-\nabla_{\xi} s$. (Note the unusual sign convention, which is necessary for consistency later.) Since $\mathcal{L}_{\xi} \nabla=0$ and $\left[\mathcal{L}_{\xi}, \mathcal{L}_{\chi}\right]=\mathcal{L}_{[\xi, \chi]}$, it follows that

$$
\mathcal{L}_{\xi}\left(S_{\chi}\right)=S_{[\xi, \chi]}
$$

and so $S: M \times \mathfrak{h} \rightarrow \mathfrak{g}_{M}$ is $H$-equivariant. Part of the curvature $F^{\nabla}$ of $\nabla$ is determined by the Higgs fields - one readily computes that for any $\xi \in \mathfrak{h}$ and any vector field $X$,

$$
F^{\nabla}(\xi, X)=\nabla_{X}\left(S_{\xi}\right) .
$$

In particular, for two vector fields $\xi, \chi$ in $\mathfrak{h}$,

$$
F^{\nabla}(\xi, \chi)=\left[S_{\xi}, S_{\chi}\right]_{\mathfrak{g}}-S_{[\xi, \chi]},
$$

where the first bracket is the Lie bracket in $\mathfrak{g}_{M}$.

Now suppose that $H$ acts freely on $M$ with nondegenerate conformal metrics on the orbits, and define $(\alpha, \varphi)$ as in Section 2. The bundle $E$ is the pullback of a bundle, also denoted $E$, over $Q$. Since $\nabla$ is $H$-invariant, it descends to a connection $A$ over $Q$. The Higgs fields are also $H$ invariant; hence setting $\Phi(U)=S_{\varphi(U)}$ defines a bundle map $\Phi: \mathcal{V} \rightarrow \mathfrak{g}_{Q}$ over $Q$. Next introduce a conformal connection $D$ on $\mathcal{V}$. Then $\nabla_{X}\left(S_{\xi}\right)=\left(D_{X}^{A} \Phi\right)\left(\varphi^{-1}(\xi)\right)-\Phi\left(\varphi^{-1}\left(D_{X}^{\alpha} \varphi\right) \varphi^{-1}(\xi)\right)$ and a simple computation of the curvature of $\nabla$ yields the following equations:

$$
\begin{aligned}
& F^{\nabla}(U, V)=[\Phi(U), \Phi(V)]_{\mathfrak{g}}-\Phi\left(\varphi^{-1}[\varphi(U), \varphi(V)]_{\mathfrak{h}}\right), \\
& F^{\nabla}(U, X)=\left(D_{X}^{A} \Phi\right)(U)-\Phi\left(\varphi^{-1} D_{X}^{\alpha} \varphi(U)\right), \\
& F^{\nabla}(X, Y)=F^{A}(X, Y)-\Phi\left(\varphi^{-1} F^{\alpha}(X, Y)\right) .
\end{aligned}
$$

This formulation makes manifest the analogy between $(A, \Phi)$ and $(\alpha, \varphi)$ : the former is a $G$ connection and $\mathfrak{g}_{Q}$-valued section of $\mathcal{V}^{*}$, while the latter is an $H$-connection and $\mathfrak{h}_{Q}$-valued section of $\mathcal{V}^{*}$.

Furthermore, these formulae for $F^{\nabla}$ are closely analogous to the formulae (2.1)-(2.3) for $T^{\mathcal{D}}$ obtained in the previous section: adding torsion terms to the above equations yields

$$
\begin{aligned}
& \left(F^{\nabla}+\Phi \circ T^{\mathcal{D}}\right)(U, V)=[\Phi(U), \Phi(V)]_{\mathfrak{g}}, \\
& \left(F^{\nabla}+\Phi \circ T^{\mathcal{D}}\right)(U, X)=\left(D_{X}^{A} \Phi\right)(U)-\Phi(\langle\mathcal{C}(U), X\rangle), \\
& \left(F^{\nabla}+\Phi \circ T^{\mathcal{D}}\right)(X, Y)=F^{A}(X, Y)-\Phi(\psi(X, Y)) .
\end{aligned}
$$

Assuming that $T^{\mathcal{D}}$ is selfdual (which can always be arranged, using the choice of $D$, by the work of Section 2), the selfduality of $F^{\nabla}$ is now equivalent to the selfduality of the right hand sides of $(4.1)-(4.3)$.

This reduced gauge field equation is easy to compute in each dimension (and equivalent calculations have already been used in Section 2). Notice that no assumption of selfduality on $M$ is needed for these computations: just as the selfdual Yang-Mills equation makes sense on any oriented conformal 4-manifold, so also the generalized Bogomolny equation is defined on any Weyl space, and the same is true on the one and two-dimensional geometries. However, 
the principal dogma underlying this work is that the natural backgrounds for the gauge field equations are selfdual spaces, Einstein-Weyl spaces, spinor-vortex spaces and Riccati spaces. There are two reasons for this: first, the Ward correspondence predicts that the reduced gauge field equations on these backgrounds will be integrable; second, it will soon be apparent, if not already, that the gauge field equations and background equations are intimately related.

\subsection{Generalized Nahm equations on Riccati spaces}

The reduction to one dimension is straightforward, since there is no curvature. Hence the connection $A$ on $\mathfrak{g}_{C}$ can be assumed trivial, and the gauge field equation for $\Phi \in \mathrm{C}^{\infty}\left(C, \mathcal{E}^{*} \otimes \mathfrak{g}_{C}\right)$ is

$$
D \Phi-*[\Phi, \Phi]_{\mathfrak{g}}=\mathcal{B} \cdot \Phi
$$

where $\mathcal{B} \cdot \Phi=\Phi \circ \mathcal{B}$ and the Lie bracket pairing $[\Phi, \Phi]_{\mathfrak{g}} \in \mathrm{C}^{\infty}\left(C, \wedge^{2} \mathcal{E}^{*} \otimes \mathfrak{g}_{C}\right)$ is interpreted as a section of $T^{*} C \otimes \mathcal{E}^{*} \otimes \mathfrak{g}_{C}$ using the Hodge star and conformal structure on $\mathcal{E}$, together with the identifications $L_{\mathcal{E}}^{1}=L_{C}^{1}=T C$. When $\mathcal{B}=0$, this is the Nahm equation.

\subsection{Generalized Hitchin equations on spinor-vortex spaces}

For the reduction to two dimensions, it is natural, as before, to reinterpret $\Phi$ as a complex linear map from $\mathcal{W}$ to $\mathfrak{g}_{N} \otimes \mathbb{C}$. Then the gauge field equations for $(A, \Phi)$ are

$$
F^{A}-[\Phi, \bar{\Phi}]_{\mathfrak{g}}=\psi \wedge \bar{\Phi}+\bar{\psi} \wedge \Phi, \quad \bar{\partial}^{a, A} \Phi=\mathcal{C} \bar{\Phi}
$$

When $\mathcal{C}=\psi=0$ and $\mathcal{W}=T N$, with the induced holomorphic structure, these are Hitchin's equations.

To adapt the gauge field equations to spinor-vortex spaces in general signature (when there are background fields $\mathcal{C}, \widetilde{\mathcal{C}}, \psi, \widetilde{\psi})$, replace $\bar{\Phi}$ by an additional field $\widetilde{\Phi}$, satisfying the analogous $\partial^{a, A}$-equation. The Lorentzian reality condition $(\Phi, \widetilde{\Phi}$ real) provides a generalized chiral model, while a generalization of the harmonic map equation is obtained by introducing the crucial sign change $\widetilde{\Phi}=-\bar{\Phi}$ (recall also that $\widetilde{\psi}=-\bar{\psi}$ in this case).

\subsection{Generalized Bogomolny equations on Einstein-Weyl spaces}

The reduction to three dimensions gives the natural generalization to Weyl geometry of the Bogomolny equation for magnetic monopoles

$$
* D^{A} \Phi=F^{A} .
$$

The Euclidean, hyperbolic or spherical Bogomolny equation arises when the Einstein-Weyl structure is given by a metric of constant curvature.

\section{Addenda: twisted flat pencils and projective pairs}

The same methodology as in the nondegenerate cases yields gauge field equations over the null reductions, using the equations (2.10)-(2.12) for the torsion $T^{\mathcal{D}}$.

In the background geometry $(a, \phi, \psi)$ on $(Q, \mathcal{L})$ obtained by reduction along an $\alpha$-surface foliation, the gauge fields consist of a $G$-connection $A$ and a 1 -form $\Phi$ with values in $\mathcal{L}^{2} \otimes \mathfrak{g}_{Q}$, and the gauge field equations are

$$
F^{A}=\psi \wedge \Phi, \quad \mathrm{d}^{a, A} \Phi=0, \quad \frac{1}{2}[\Phi \wedge \Phi]=\chi \wedge \Phi .
$$


If $\psi=\chi=0$ (so $a$ is flat, and $\mathcal{L}$ may be trivialized) then these are the equations for a pencil of flat connections $\mathrm{d}^{A}+\lambda \Phi$ on a surface (also known as the topological chiral model). Thus solutions of (4.5) may be called twisted flat pencils. The special case $\psi=0$ has been studied by Tafel and Wójcik in [52].

On a projective surface $(Q,[D])$ obtained by reduction along a $\beta$-surface foliation, the gauge fields consist of a $G$-connection $A$ and a 1 -form $\Phi$ with values in $\mathcal{O}_{Q}(2) \otimes \mathfrak{g}_{Q}$ (where $\mathcal{O}_{Q}(3)=$ $\left.\wedge^{2} T Q\right)$, and the gauge field equations are

$$
D^{A} \Phi=\frac{1}{2} \mathrm{~d}^{D, A} \Phi
$$

Solutions $(A, \Phi)$ were referred to (somewhat unimaginatively) as projective pairs in [65].

The special case of reductions of the selfdual Yang-Mills equation on $\mathbb{R}^{2,2}$ (or $\mathbb{C}^{4}$ ) by null translations was considered already by Mason and Woodhouse [41]: the $\alpha$-plane reduction (yielding flat pencils) is denoted $H_{S D}$, while the $\beta$-plane reduction (yielding projective pairs) is denoted $H_{A S D}$.

\section{Interlude: spinor-vortex spaces and Hitchin's equations}

In [30], Hitchin considered solutions of the selfdual Yang-Mills equation on $\mathbb{R}^{4}$ invariant under two translations. He observed that the Yang-Mills connection could be decomposed into a connection over $\mathbb{R}^{2}$ and two Higgs fields. He combined these Higgs fields into a complex Higgs field and then noticed that, remarkably, the reduced Yang-Mills equation becomes conformally invariant provided this complex Higgs field is interpreted as a 1-form rather than a scalar. This was a surprise, because although the selfdual Yang-Mills equation is conformally invariant, the notion of translation-invariance is not. Furthermore, conformal invariance in two dimensions implies an infinite-dimensional symmetry group.

The reduction process described here provides a simple explanation of this phenomenon: $W^{-}=0$ is conformally invariant, and so is the notion of torus symmetry. Hence the equations for $\left(\bar{\partial}^{a}, \psi, \mathcal{C}, \alpha, \varphi\right)$ are conformally invariant on a fixed Riemann surface with a complex line bundle $\mathcal{W}$ and a Hermitian metric on $\mathcal{W}^{-1} T N$. In particular, it is clear that the equations (2.6)(2.8) for $\left(\bar{\partial}^{a}, \psi, \mathcal{C}\right)$ are conformally invariant.

If $\mathcal{C}$ is not identically zero, then (on the open set where $\mathcal{C}$ is nonvanishing) the freedom in the holomorphic structure on $\mathcal{W}$ can be fixed by declaring that $\mathcal{C}$ is an identification of $\mathcal{W}^{2}$ with $T N$. The length of $\mathcal{C}$ now defines a gauge, breaking conformal invariance. More precisely, given a Weyl derivative $D$, the Chern connection on $\mathcal{W}$ only agrees with the connection induced from $T N$ if $D=D^{g}$, where $g$ is the metric induced by $|\mathcal{C}|$. Equation (2.8) becomes $\frac{1}{2} s^{g}=-2+4|\psi|_{g}^{2}$; in particular, if $\psi$ is zero, then $g$ has constant negative curvature, which is the case studied by Joyce [37], the hyperbolic spinor-vortex geometry.

On the other hand if $\mathcal{C}$ is identically zero, then $\psi$ is holomorphic, so if $\psi$ is not identically zero, then (on the open set where $\psi$ is nonvanishing), $\psi$ trivializes $\mathcal{W}$. Again this breaks conformal invariance by introducing a natural gauge, $|\psi|$; the corresponding metric has constant positive curvature $\left(s^{g}=4\right)$. This is the spherical spinor-vortex geometry.

Finally if $\mathcal{C}$ and $\psi$ both vanish, then the holomorphic structure can be fixed by setting $\mathcal{W}=$ $T N$, so that the Chern connection on $\mathcal{W}^{-1} T N$ is trivial. This trivial spinor-vortex geometry does not break conformal invariance.

The Yang-Mills equation is reduced to two dimensions by interpreting the Higgs fields as a section $\Phi$ of $\mathcal{W}^{-1} \otimes_{\mathbb{R}} \operatorname{End}(V)$, using $\varphi$. The resulting gauge field equations are independent of $(\alpha, \varphi)$, i.e., they are intrinsic to the spinor-vortex space. On a trivial spinor-vortex space, $\mathcal{W}=T N$, so $\Phi$ becomes an endomorphism-valued 1-form, and, as remarked already, the gauge field equations for $(A, \Phi)$ are Hitchin's equations. Thus it is the isomorphism $\varphi$, and the geometry 
of the trivial spinor-vortex space that are responsible for the interpretation of the Higgs fields as a 1-form, rather than as scalars.

\section{Selfdual spaces from gauge fields}

In Sections 2 and 4, background geometries and gauge field equations were found by reducing the selfduality equation for conformal structures and Yang-Mills fields respectively. In the approach taken, the symmetry group $H$, and the pair $(\alpha, \varphi)$ defining the reduction, decoupled from the construction, and the reduced background geometry and gauge field equations were found to be independent of these data.

In some sense, this was a sleight of hand, since $(\alpha, \varphi)$ were used to define the data on the quotient which make up the background geometry (i.e., $D, \psi$ and $\mathcal{C}$ ). However, the procedure may be turned around: starting with the background geometry, these definitions, originally obtained by imposing selfduality on the torsion $T^{\mathcal{D}}$, may be viewed as equations for the pair $(\alpha, \varphi)$. Indeed, applying $\varphi$ to the formulae $(2.1)-(2.3)$ for $T^{\mathcal{D}}$ gives:

$$
\begin{aligned}
& \left(\varphi \circ T^{\mathcal{D}}\right)(U, V)=[\varphi(U), \varphi(V)]_{\mathfrak{h}}, \\
& \left(\varphi \circ T^{\mathcal{D}}\right)(U, X)=\left(D_{X}^{\alpha} \varphi\right)(U)-\varphi(\langle\mathcal{C}(U), X\rangle), \\
& \left(\varphi \circ T^{\mathcal{D}}\right)(X, Y)=F^{\alpha}(X, Y)-\varphi(\psi(X, Y)) .
\end{aligned}
$$

The right hand sides of these formulae correspond precisely to the right hand sides of (4.1)-(4.3). Hence the selfduality equation for $T^{\mathcal{D}}$ coincides with the gauge field equation for $(\alpha, \varphi)$ on the background geometry.

This leads immediately to an inverse construction of selfdual spaces with symmetry from gauge fields with $\ell$-dimensional gauge group on $k$-dimensional background geometries, where $k+\ell=4$. However, the construction can be generalized further by noting that the selfduality of $T^{\mathcal{D}}$ is implied by the gauge field equation as long as $\varphi: \mathcal{V} \rightarrow \mathfrak{h}_{Q}$ is injective. I will now explain this generalized construction.

Let $P \rightarrow Q$ be a principal $H$-bundle with an $H$-connection $\alpha$ and a Higgs field $\varphi: \mathcal{V} \rightarrow \mathfrak{h}_{Q}$, where $\mathfrak{h}_{Q}=P \times_{H} \mathfrak{h}$. Suppose that $H$ acts transitively on an $\ell$-manifold $\Sigma^{\ell}$, where $Q$ has dimension $k=4-\ell$, so that the associated fibre bundle $P \times_{H} \Sigma^{\ell}$ is four-dimensional.

The basic example is the case that the action of $H$ is also free, in which case $\Sigma^{\ell}$ is a principal homogeneous space for $H$ and therefore there is a commuting free transitive action of a Lie group $\tilde{H}$ isomorphic to $H$. Choosing a basepoint on $\Sigma^{\ell}$ identifies $\tilde{H}$ and $\Sigma^{\ell}$ with $H$ and the two actions are the left and right regular actions. However, it can be useful to distinguish between the structure group $H$, and the symmetry group $\tilde{H}$ : if $P$ is a principal $H$-bundle over $Q$, then $P \times_{H} \Sigma^{\ell}$ is a principal $\tilde{H}$-bundle.

For general $\Sigma^{\ell}$, there is still an associated bundle $\pi: P \times_{H} \Sigma^{\ell} \rightarrow Q$, but this does not have any symmetries in general, since there is no longer a commuting right action of $\tilde{H}$ on $\Sigma^{\ell}$. However, the fibre of $\mathfrak{h}_{Q}=P \times_{H} \mathfrak{h}$ is still a Lie algebra of vertical vector fields on $P \times_{H} \Sigma^{\ell}$, which will be called "invariant", but for consistency with the case $\Sigma^{\ell}=H$, the Lie bracket of these vector fields is opposite to the Lie bracket in $\mathfrak{h}_{Q}$. The map $\varphi: \mathcal{V} \rightarrow \mathfrak{h}_{Q}$ therefore induces a map $\widehat{\varphi}$ from $\pi^{*} \mathcal{V}$ to the vertical bundle of $P \times_{H} \Sigma^{\ell}$. These bundles both have rank $\ell$, so let $M$ be an open subset of $P \times_{H} \Sigma^{\ell}$ where $\widehat{\varphi}$ is an isomorphism. Note that $\widehat{\varphi}$ sends basic sections to "invariant" vector fields.

Equip $T M \cong\left(\left.\pi\right|_{M}\right)^{*}(\mathcal{V} \oplus T Q)$ with the direct sum conformal structure, so that a conformal connection $D$ on $\mathcal{V}$ induces a conformal connection on $T M$. As before, a modified conformal connection $\mathcal{D}$ can be constructed, using the pullbacks of $\psi$ and $\mathcal{C}$ : the torsion of this connection will be vertical-valued, and will satisfy (6.1)-(6.3). Since $\varphi$ is injective, the torsion will be selfdual if $(\alpha, \varphi)$ satisfy the gauge field equation on $Q$. The calculation of the curvature of $\mathcal{D}$ 
carries over immediately to this more general setting, hence Joyce's Lemma can be applied to establish the following result.

Theorem 6.1. Let $H$ be a transitive group of diffeomorphisms of an $\ell$-manifold $\Sigma^{\ell}$. Suppose that $(\alpha, \varphi)$ is a solution of the gauge field equation (i.e., the Nahm, Hitchin or Bogomolny equation) on a principal $H$-bundle $P \rightarrow Q$, where $Q$ is a $(4-\ell)$-dimensional background geometry (i.e., an Einstein-Weyl, spinor-vortex or Riccati space). Then on the open subset $M$ of $\pi: P \times_{H} \Sigma^{\ell} \rightarrow Q$ where $\widehat{\varphi}$ is an isomorphism, $(\alpha, \varphi)$ identifies $T M$ with $\pi^{*}(\mathcal{V} \oplus T Q)$, and the direct sum conformal structure is selfdual.

For later work, it will be useful to have a more explicit description of the construction of this theorem. Choose a local conformal frame $e_{i}$ for $T Q \oplus \mathcal{V}$ over $Q$ compatible with the direct sum decomposition, and a local section of $P$. Then, by identifying $M$ locally with $Q \times \Sigma^{\ell}$ and viewing the connection $\alpha$ as a $\operatorname{Vect}\left(\Sigma^{\ell}\right)$-valued 1-form on $Q$, the components of $(\alpha, \varphi)$ with respect to $e_{i}$ define four vector fields $X_{i}$ on $\Sigma^{\ell}: X_{1}, \ldots X_{k}$ are the components of the connection and $X_{k+1}, \ldots X_{4}$ are the components of $\varphi$. Since $e_{i}$ is a conformal frame on $T Q \oplus \mathcal{V}$, the conformal structure on $M$ is clearly represented contravariantly by the metric

$$
\left(e_{1}-X_{1}\right)^{2}+\cdots+\left(e_{k}-X_{k}\right)^{2}+X_{k+1}^{2}+\cdots+X_{4}^{2} .
$$

This is a metric on $T^{*} M$. The covariant metric on $T M$ is dual to this, and will be discussed in Section 8. In fact it is sometimes more convenient to use contravariant metrics, since they push forward easily.

\section{Remarks 6.2.}

(i) Note that the calculations leading to this theorem are entirely formal and so, at least for local considerations, $H$ need not be finite-dimensional, but could be any subgroup of $\operatorname{Diff}\left(\Sigma^{\ell}\right)$. Hence the conformal aspects of Ward's construction [62] are included in the theorem when the background geometry is trivial.

(ii) Theorem 6.1 provides a new interpretation of the switch map [41, 43]. Let $M$ be a selfdual conformal 4-manifold with freely acting $\ell$-dimensional symmetry group $H$. Then the local quotient $Q=M / H$ is an background geometry of dimension $4-\ell$. An invariant selfdual Yang-Mills field (on a principal bundle $P$ ) with $\ell$-dimensional gauge group $G$ descends to a solution of the gauge field equation on $Q$, from which a new selfdual space $\tilde{M}$ may be constructed.

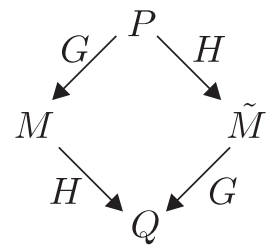

Note the following features of this interpretation.

- The construction avoids considering the $(4+\ell)$-dimensional manifold $P$ explicitly.

- The procedure decomposes into two steps of independent interest: the construction of gauge fields on $Q$ from $H$-invariant selfdual Yang-Mills fields on $M$, and the construction of selfdual spaces from gauge fields on $Q$.

- The group $G$ need not be $\ell$-dimensional, so long as it acts transitively on an $\ell$ manifold. Hence the constructions of [19] fit into the same framework.

(iii) In the case that $\Sigma^{\ell}=H, M$ has symmetry group $H$, but enlarging the group $H$ acting on $\Sigma^{\ell}$ gives less symmetry, rather than more symmetry, since there are fewer diffeomorphisms 
of $\Sigma^{\ell}$ commuting with the $H$-action. In general there will be none, and $M$ will have no symmetry. In other words, the group $H$ is a structure group rather than a symmetry group: only in the case of $H$ acting freely does it happen that the commuting symmetry group is (isomorphic to) $H$.

Selfdual conformal manifolds with symmetry groups which do not act freely are rather special: for instance they are foliated by the surfaces of equal isotropy group, and the curvature must be invariant under the isotropy representation. Some examples arise as very special cases of the constructions of this paper. More precisely, if $G$ is a (not necessarily free or transitive) group of diffeomorphisms of $\Sigma^{\ell}$, then $G$ will be a symmetry group of $M$ provided there is a representation of $G$ on $\mathcal{V}$ such that $\varphi$ and $\alpha$ are $G$-invariant. In the case that $G$ acts trivially on $\mathcal{V}$, this means that the structure group $H$ reduces to the group of diffeomorphisms of $\Sigma^{\ell}$ commuting with $G$. On the other hand, there are interesting examples where $G$ acts nontrivially on $\mathcal{V}$, see for instance [33].

The use of structure groups rather than symmetry groups also turns out to be natural when partial reductions are considered in view of the following remark. If $H$ acts freely on $M$ and $K$ is a subgroup of $H$, then $K$ also acts freely on $M$ and the structure group of $M / K$ is $H / K_{0}$ acting on $H / K$, where $K_{0}$ is the maximal normal subgroup of $H$ lying in $K$. For example, if $\mathrm{SU}(2)$ acts freely on $M$ then $\mathrm{SO}(3)$ acting on $S^{2}$ will be the induced structure group for $M / \mathrm{U}(1)$. In Section 8 , it will be shown that such partial reductions $M / K$ arise directly from gauge fields on $M / H$ with gauge group $H / K_{0}$.

\section{Addenda: generalized constructions from null reductions}

The same principles may be applied to null reductions to obtain constructions of selfdual 4manifolds from twisted flat pencils and projective pairs, where the gauge group is a transitive group of diffeomorphisms of a surface $\Sigma^{2}$. The only change needed, relative to the nondegenerate case, is that the conformal structure on $M$ is obtained from the natural pairing between $V M$ and $\pi^{*} T Q$, rather than conformal structures on each summand.

The construction of selfdual conformal structures from flat pencils of connections on a surface is not at all new: as discussed in the following Interlude, it underpins Plebanski's heavenly equations and interpretations of hypercomplex and hyperkähler structures as topological chiral models. On the other hand, the construction of selfdual conformal structures from twisted flat pencils with gauge group $\operatorname{Diff}\left(\Sigma^{2}\right)$ has not been studied, as far as I am aware.

The analogous story for projective pairs on a projective surface underpins the DunajskiWest construction of selfdual conformal manifolds with a null Killing vector [72]. In [65], their construction is shown to be a reduction of gauge group from $\operatorname{Diff}\left(\Sigma^{2}\right)$ to the subgroup commuting with a nonvanishing vector field.

\section{Interlude: hypercomplex and hyperkähler structures}

From the point of view of integrable systems, hypercomplex and hyperkähler 4-manifolds are considerably simpler than the general selfdual space.

Recall that a hypercomplex structure consists of a triple $I, J, K$ of integrable complex structures, satisfying the quaternionic relation $I J=K$. It is well known that a hypercomplex manifold comes equipped with a unique torsion-free connection $D$ with $D I=D J=D K=0$, called the Obata connection [45].

A hypercomplex 4-manifold possesses a canonical conformal structure, defined by requiring that $(X, I X, J X, K X)$ is a conformal frame for any nonzero tangent vector $X$. The Obata connection preserves this conformal structure, and is thus a Weyl connection. Since $I, J, K$ 
are anticommuting orthogonal complex structures, their (weightless) Kähler forms are either all selfdual or all antiselfdual: I fix the orientation by requiring that they are antiselfdual. Therefore $D$ is flat on $L^{2} \wedge_{-}^{2} T^{*} M$, and in particular, $M$ is a selfdual conformal manifold. The hypercomplex structure is hyperkähler if and only if $D$ is exact, i.e., the Obata connection preserves a length scale, and hence a metric in the conformal class.

The simplicity of the hypercomplex condition manifests itself in the following local description of hypercomplex 4-manifolds.

Theorem 7.1. Let $V_{0}, V_{1}, V_{2}, V_{3}$ be linearly independent vector fields on a 4-manifold $M$ and let $\eta_{0}, \eta_{1}, \eta_{2}, \eta_{3}$ be the dual coframe of 1 -forms $\left(\right.$ with $\left.\eta_{i}\left(V_{j}\right)=\delta_{i j}\right)$. Define almost complex structures $I, J, K$ by

$$
I V_{0}=V_{1}, \quad I V_{2}=V_{3}, \quad J V_{0}=V_{2}, \quad J V_{3}=V_{1}, \quad K V_{0}=V_{3}, \quad K V_{1}=V_{2} .
$$

Then the following are equivalent.

1. The frame $V_{i}$ satisfies the equations

$$
\begin{aligned}
& {\left[V_{0}, V_{1}\right]+\left[V_{2}, V_{3}\right]=0,} \\
& {\left[V_{0}, V_{2}\right]+\left[V_{3}, V_{1}\right]=0,} \\
& {\left[V_{0}, V_{3}\right]+\left[V_{1}, V_{2}\right]=0 .}
\end{aligned}
$$

2. For each $i, \mathrm{~d} \eta_{i}$ is selfdual with respect to the conformal structure represented by the metric $g=\eta_{0}^{2}+\eta_{1}^{2}+\eta_{2}^{2}+\eta_{3}^{2}$ (where $I, J, K$ are antiselfdual).

3. $(I, J, K)$ is hypercomplex with Obata connection $D$ and $\operatorname{div}^{D} \eta_{i}=0$ for all $i$.

Any hypercomplex 4-manifold $M$ arises locally in this way, and is hyperkähler if and only if the vector fields $V_{i}$ all preserve a volume form $\nu$.

The hyperkähler version of this theorem is due to Mason-Newman [40], following a construction of Ashtekar-Jacobson-Smolin [2] which will be described below. The hyperkähler case is simpler, because for $D$ exact, the $\eta_{i}$ are divergence-free with respect to $D$ if and only if the $V_{i}$ preserve a volume form - see (7.4).

The general construction was first written explicitly by Joyce [37], but the fact that all fourdimensional hypercomplex structures arise in this way is due to Dunajski [17]. The description I have given differs slightly from these references, and owes a great deal to the approaches of Hitchin [33] (see below) and Grant-Strachan [26]. Since the role of the divergence condition has perhaps not been fully elucidated before, and will be useful later, I give a complete proof.

Proof. Since

$$
\eta_{i}\left(\left[V_{j}, V_{k}\right]\right)=-\mathrm{d} \eta_{i}\left(V_{j}, V_{k}\right)
$$

for all $i, j, k$, it is manifest that (i) and (ii) are equivalent formulations of the same equations. Also, (7.2) and (7.3) clearly imply that $I$ is integrable since they may be rewritten as

$$
\left[V_{0}+\boldsymbol{i} V_{1}, V_{2}+\boldsymbol{i} V_{3}\right]=\left[V_{0}, V_{2}\right]-\left[V_{1}, V_{3}\right]+\boldsymbol{i}\left(\left[V_{0}, V_{3}\right]+\left[V_{1}, V_{2}\right]\right)=0
$$

Similarly (7.3) and (7.1) imply that $J$ is integrable, and (7.1) and (7.2) imply that $K$ is integrable. Now note that for any 1-form $\eta$ on a hypercomplex manifold $\mathrm{d} \eta$ is selfdual if and only if it is orthogonal to the weightless Kähler forms of $I, J, K$. Since $D I=D J=D K=0$, this is equivalent to $I \eta, J \eta$ and $K \eta$ being divergence-free with respect to $D$ (for instance, $\operatorname{div} D(I \eta)=$ $\sum_{i}\left\langle\varepsilon^{i}, I D_{e_{i}} \eta\right\rangle$, and $I$ is skew). Hence (iii) is equivalent to (i) and (ii). 
On any hypercomplex manifold, the conditions $\operatorname{div}^{D} \eta=0$ and $\mathrm{d} \eta^{-}=0$ form a determined first-order linear system for a 1-form $\eta$, which therefore admits local (non-null) solutions: $(\eta, I \eta, J \eta, K \eta)$ is then a divergence-free conformal coframe.

It remains to characterize the hyperkähler case in terms of volume forms - or length scales. Suppose that $\mu=e^{f} \mu_{g}$ is a length scale; then $\mu^{-4} V_{i}=\mu^{-4} \mu_{g}^{2} \eta_{i}=e^{-2 f} \mu^{-2} \eta_{i}$ and so

$$
\operatorname{div}\left(\mu^{-4} V_{i}\right)=\operatorname{div}^{D}\left(e^{-2 f} \mu^{-2} \eta_{i}\right)=\left\langle D\left(e^{-2 f} \mu^{-2}\right), \eta_{i}\right\rangle+e^{-2 f} \mu^{-2} \operatorname{div}^{D} \eta_{i} .
$$

Since $\operatorname{div}^{D} \eta_{i}=0, D$ preserves the length scale $e^{f} \mu=e^{2 f} \mu_{g}$ if and only if the $V_{i}$ all preserve the volume form $\nu=\mu^{-4}$ (note that $\wedge^{4} T^{*} M \cong L^{-4}$, using the orientation of $M$ ).

There is an equivalent way to describe the divergence-free condition on the coframe, using spinors $[17,41]$. Recall that any conformal 4-manifold locally admits (weightless) spin bundles $\$^{ \pm}$, which are $\mathrm{SL}(2, \mathbb{C})$ bundles such that $\$^{+} \otimes \$^{-}$is isomorphic to the complexified weightless cotangent bundle $L T^{*} M \otimes \mathbb{C}$ with the metric induced by the two area forms. The conventions are chosen so that $L^{2} \wedge_{-}^{2} T^{*} M \otimes \mathbb{C}=S^{2} \$^{-}$. On a hypercomplex manifold $D$ induces a flat connection on $\$^{-}$preserving the area form.

For Euclidean reality conditions, the real structure on the $L T^{*} M \otimes \mathbb{C}$ is induced by (parallel) quaternionic structures on $\$^{ \pm}$. If frames $\left(\rho_{0}, \rho_{1}\right)$ for $L^{-1} \$^{+}$and $\left(\sigma_{0}, \sigma_{1}\right)$ for $\$^{-}$are chosen so that the quaternionic structure sends $\rho_{0}$ to $\rho_{1}$ and $\sigma_{0}$ to $\sigma_{1}$, then

$$
\begin{aligned}
& \eta_{0}+\boldsymbol{i} \eta_{1}=\rho_{0} \otimes \sigma_{0}, \quad \eta_{2}+\boldsymbol{i} \eta_{3}=\rho_{0} \otimes \sigma_{1}, \\
& \eta_{0}-\boldsymbol{i} \eta_{1}=\rho_{1} \otimes \sigma_{1}, \quad \eta_{2}-\boldsymbol{i} \eta_{3}=-\rho_{1} \otimes \sigma_{0}
\end{aligned}
$$

defines a real conformal coframe $\left(\eta_{0}, \eta_{1}, \eta_{2}, \eta_{3}\right)$.

Proposition 7.2. Suppose that $M$ is hypercomplex with Obata connection $D$ and that $\left(\sigma_{0}, \sigma_{1}\right)$ is a D-parallel frame for $\$^{-}$.

1. Let $\rho$ be a section of $L^{-1} \$^{+}$. Then $\rho \otimes \sigma_{0}$ and $\rho \otimes \sigma_{1}$ are divergence-free with respect to $D$ if and only if $\rho$ satisfies the Dirac-Weyl equation $\sum_{i} \varepsilon_{i} \cdot D_{e_{i}} \rho=0$.

2. Let $\eta$ be a 1-form. Then $\eta \cdot \sigma_{0}$ and $\eta \cdot \sigma_{1}$ satisfy the Dirac-Weyl equation if and only if $\operatorname{div}^{D} \eta=0$ and $\mathrm{d} \eta^{-}=0$.

Here the dot denotes the natural (Clifford) action $T^{*} M \otimes L^{w} \$^{ \pm} \rightarrow L^{w-1} \$^{\mp}$.

Proof. These are direct calculations:

(i) For $A=0,1, \operatorname{div}^{D}\left(\rho \otimes \sigma_{A}\right)=\sum_{i} \varepsilon^{i}\left(D_{e_{i}} \rho \otimes \sigma_{A}\right)=\omega^{-}\left(\sum_{i} \varepsilon^{i} \cdot D_{e_{i}} \rho, \sigma_{A}\right)$.

(ii) For $A=0,1, \sum_{i} \varepsilon^{i} \cdot D_{e_{i}}\left(\eta \cdot \sigma_{A}\right)=\sum_{i} \varepsilon^{i} \cdot\left(D_{e_{i}} \eta\right) \cdot \sigma_{A}=\left(\operatorname{div}^{D} \eta\right) \sigma_{A}+\mathrm{d} \eta \cdot \sigma_{A}$.

In (i) $\omega^{-}$is the area form on $\$^{-}$, and in (ii) the Clifford action of $\mathrm{d} \eta^{+}$is trivial.

An important class of solutions to the equations $\operatorname{div}^{D} \eta=0$ and $\mathrm{d} \eta^{-}=0$ is obtained by taking $\eta=\mathrm{d} r$ where $\Delta^{D} r:=\operatorname{div}^{D} \mathrm{~d} r=0$. Evidently the equation $\Delta^{D} r=0$ admits local solutions on any hypercomplex 4-manifold. Hence the following result is obtained, which is the original construction of Ashtekar-Jacobson-Smolin [2] in the hyperkähler case, and is due to Hitchin [33] in general.

Theorem 7.3. Let $\Phi$ be a solution of the Nahm equation (on a trivial Riccati space) with gauge group $\operatorname{Diff}\left(\Sigma^{3}\right)$ for some 3 -manifold $\Sigma^{3}$. Then the selfdual space constructed from $\Phi$ is hypercomplex. Any hypercomplex 4-manifold arises locally in this way, and is hyperkähler if and only if there is a reduction to the gauge group $\operatorname{SDiff}\left(\Sigma^{3}\right)$. 
Proof. Let $r$ be an affine coordinate on a trivial Riccati space $C$, choose a conformal trivialization of $\mathcal{E}$, and write the components of $\Phi$, which are vector fields on $\Sigma^{3}$, as $\left(V_{1}, V_{2}, V_{3}\right)$. Then the vector fields $\left(\partial_{r}, V_{1}, V_{2}, V_{3}\right)$ on $M \subset C \times \Sigma^{3}$ satisfy (7.1)-(7.3) as a consequence of the Nahm equation, and so the dual frame $\left(\mathrm{d} r, \eta_{1}, \eta_{2}, \eta_{3}\right)$ has $\mathrm{d} \eta_{i}$ selfdual (note that I use the opposite orientation to [33]). Conversely any hypercomplex manifold arises locally in this way by letting $r$ be a solution of $\Delta^{D} r=0$ and setting $\left(\eta_{1}, \eta_{2}, \eta_{3}\right)=(I \mathrm{~d} r, J \mathrm{~d} r, K \mathrm{~d} r)$.

Finally note that $\partial_{r}$ and $V_{i}$ preserve a volume form $e^{-4 f} \mathrm{~d} r \wedge \eta_{1} \wedge \eta_{2} \wedge \eta_{3}$ if and only if

$$
\mathcal{L}_{\partial_{r}}\left(e^{-4 f} \eta_{1} \wedge \eta_{2} \wedge \eta_{3}\right)=0 \quad \text { and } \quad \mathrm{d} r \wedge \mathcal{L}_{V_{i}}\left(e^{-4 f} \eta_{1} \wedge \eta_{2} \wedge \eta_{3}\right)=0
$$

Here I use the fact that $\mathrm{d} r\left(V_{i}\right)=0, \mathrm{~d} r\left(\partial_{r}\right)=1$ and that $\iota_{\partial_{r}}\left(e^{-4 f} \eta_{1} \wedge \eta_{2} \wedge \eta_{3}\right)=0$. The first equation says that $e^{-4 f} \eta_{1} \wedge \eta_{2} \wedge \eta_{3}$ is an $r$-independent volume element on $\Sigma^{3}$ (i.e., a parallel volume form on $C \times \Sigma^{3} \rightarrow C$ ), and the second equation says that the $V_{i}$ are volume-preserving vector fields for each fixed $r$ (i.e., on each fibre of $C \times \Sigma^{3} \rightarrow C$ ). This is exactly what it means to have a reduction to $\operatorname{SDiff}\left(\Sigma^{3}\right)$.

As this construction involves taking $\mathrm{d} \eta_{0}=0$, it is natural to ask if one can find divergencefree coframes with $\mathrm{d} \eta_{0}=0=\mathrm{d} \eta_{1}$ and hence formulate the hypercomplex equations as Hitchin equations on a trivial spinor-vortex space.

A number of constructions of four-dimensional hyperkähler metrics from two-dimensional integrable models are known, due to Park [46], Ward [62] and (later) Husain [34]: see [58] for a review. Unfortunately, it is not always clear in these constructions whether all hyperkähler metrics are obtained, what choices are needed to obtain the integrable model from a hyperkähler metric, and if they are compatible with Euclidean reality conditions. In particular, as far as I can tell, none of these works establish an equivalent formulation of the Euclidean hyperkähler condition as a Euclidean two-dimensional integrable model.

Indeed, the usual approach is to use the equation $\left[V_{0}+\boldsymbol{i} V_{1}, V_{2}+\boldsymbol{i} V_{3}\right]=0$ to introduce coordinates $(x, y, u, v)$ such that $V_{0}+\boldsymbol{i} V_{1}=\partial_{x}$ and $V_{2}+\boldsymbol{i} V_{3}=\partial_{y}$. There are several variations on this theme, since the meaning of $\partial_{x}$ and $\partial_{y}$ in $(x, y, u, v)$ coordinates depends on $u$ and $v$, leading to different forms for $V_{0}-\boldsymbol{i} V_{1}$ and $V_{2}-i V_{3}$. This procedure tends to obscure the nature of the choice made to obtain the frame, making it more difficult to argue that any hyperkähler metric admits such a frame.

Fortunately there is an alternative approach, which clarifies the choice of frame, is easily made compatible with any reality conditions, and generalizes to the hypercomplex case. The following elementary observation is very well known, at least in the hyperkähler case.

Proposition 7.4. Let $z$ be a complex function on a hypercomplex 4-manifold $(M, D)$ which is holomorphic with respect to one of the complex structures. Then $\Delta^{D} z=0$, so that $\mathrm{d} z$ is a complex null 1-form which is divergence-free with respect to $D$.

Proof. If $z$ is $I$-holomorphic, then $I \mathrm{~d} z:=-\mathrm{d} z \circ I=-\boldsymbol{i} \mathrm{d} z$. Now $I$ is skew and $D I=0$, so $\operatorname{div}^{D}(I \mathrm{~d} z)=\sum_{i}\left\langle\varepsilon^{i}, I D_{e_{i}} \mathrm{~d} z\right\rangle=0$ since $\mathrm{d}^{2} z=0$. Hence $\Delta^{D} z=\operatorname{div}^{D}(\mathrm{~d} z-\boldsymbol{i} I \mathrm{~d} z)=0$.

Remark 7.5. I have presented this observation using language adapted to Euclidean signature manifolds. In Kleinian signature, some of the complex structures are imaginary, so that $\boldsymbol{i} I$ (say) is a real involution, inducing a decomposition $T M=T^{+} M \oplus T^{-} M$ into its \pm 1 eigenspaces. These distributions are integrable, and the analogue of a holomorphic function is a function constant on one of the families of integral surfaces - such functions can of course be real valued. Note that the orientation 2-forms of these integral surfaces are the (weightless) Kähler forms of the null complex structures $J \pm \boldsymbol{i} K$, which are decomposable (and up to rotation, $J$ and $\boldsymbol{i} K$ are real). 
The Euclidean and Kleinian cases can be considered together by first working on a complexified hypercomplex manifold, then imposing reality conditions. The above proposition applies equally in the complexified setting. Other, more Kleinian, arguments are also available (cf. $[13,50])$.

Before discussing the non-null reduction to two dimensions, I will briefly discuss the null reduction (closely related to Plebanski's heavenly equations [50]) which is used in the literature to relate the selfdual vacuum equation to a topological chiral model [46, 62]. I present the generalizations to the hypercomplex case, following $[17,26]$.

\section{Topological models and heavenly equations}

Choose independent functions $(w, z)$, both holomorphic with respect to $I$; then $\mathrm{d} w, \mathrm{~d} z$ are null 1 -forms with $\langle\mathrm{d} w, \mathrm{~d} z\rangle=0$, and one can take these to be $\eta_{0}+\boldsymbol{i} \eta_{1}$ and $\eta_{2}+\boldsymbol{i} \eta_{3}$. This can be done compatibly with Kleinian reality conditions by taking $\boldsymbol{i} I$ and $(w, z)$ real, but is incompatible with Euclidean reality conditions.

Locally, $M$ is a bundle of null surfaces over a quotient surface $N$ with coordinates $w, z$. Choosing fibre coordinates amounts to choosing a local trivialization of this bundle, and locally one can take $M=N \times \Sigma^{2}$. Then $V_{0}-\boldsymbol{i} V_{1}=\partial_{w}-\alpha, V_{2}-\boldsymbol{i} V_{3}=\partial_{z}-\beta, V_{0}+\boldsymbol{i} V_{1}=\phi, V_{2}+\boldsymbol{i} V_{3}=\psi$, where $\alpha, \beta, \phi, \psi$ are vector fields tangent to the fibres. The equations (7.1)-(7.3) now read:

$$
\left[\partial_{w}-\alpha, \partial_{z}-\beta\right]=0, \quad[\phi, \psi]=0, \quad\left[\partial_{z}-\alpha, \phi\right]+\left[\partial_{w}-\beta, \psi\right]=0,
$$

which are the equations for a pencil of flat connections $\mathrm{d}+A+\lambda \Phi$ with gauge group $\operatorname{Diff}\left(\Sigma^{2}\right)$, where $A=-\alpha \mathrm{d} w-\beta \mathrm{d} z$ and $\Phi=-\psi \mathrm{d} z+\phi \mathrm{d} w$. (This is also known as a topological chiral or sigma model.)

Plebanski's first and second heavenly equations [50], and their generalizations to the hypercomplex case (due to Grant-Strachan [26] and Dunajski [17] respectively) are obtained by fixing the gauge freedom in different ways.

- First, since $\mathrm{d}+A$ is flat, one can set $A=0$ (i.e., $\alpha=\beta=0$ ), then integrate the equation $\mathrm{d} \Phi=0$ (i.e., $\phi_{z}+\psi_{w}=0$ ) to get $V_{0}-\boldsymbol{i} V_{1}=\partial_{w}, V_{2}-\boldsymbol{i} V_{3}=\partial_{z}, V_{0}+\boldsymbol{i} V_{1}=U_{w}, V_{2}+\boldsymbol{i} V_{3}=-U_{z}$ for a vector field $U$ tangent to the fibres (with $U_{z}$ and $U_{w}$ linearly independent). The remaining equation is $\left[U_{w}, U_{z}\right]=0$. If $U$ is area preserving on the fibres, with local hamiltonian $\Omega$, then Plebanski's first equation $\left\{\Omega_{w}, \Omega_{z}\right\}=1$ is obtained, where $\{\cdot, \cdot\}$ denotes the Poisson bracket with respect to a suitably scaled area form on the fibres.

- Second, since $[\phi, \psi]=0$, one can choose the fibre coordinates $(x, y)$ so that $\phi=\partial_{x}$ and $\psi=-\partial_{y}$, then integrate the equation $\alpha_{x}-\beta_{y}=0$ to give $V_{0}-\boldsymbol{i} V_{1}=\partial_{w}-\gamma_{y}, V_{2}-\boldsymbol{i} V_{3}=\partial_{z}-\gamma_{x}$, $V_{0}+\boldsymbol{i} V_{1}=\partial_{x}, V_{2}+\boldsymbol{i} V_{3}=-\partial_{y}$. The remaining equation is $\gamma_{x w}-\gamma_{y z}=\left[\gamma_{x}, \gamma_{y}\right]$. Again the area preserving condition reduces everything to a single function $\Theta$, satisfying Plebanski's second heavenly equation $\Theta_{x w}+\Theta_{y z}=\left\{\Theta_{x}, \Theta_{y}\right\}$.

All complexified hypercomplex and hyperkähler metrics are obtained from these constructions, but information about Euclidean real slices is lost.

\section{Hypercomplex structures from the Hitchin equations}

In order to obtain a formulation compatible with Euclidean reality conditions, take $z$ to be $I$-holomorphic and $\tilde{z}$ to be $(-I)$-holomorphic. Then $\mathrm{d} z$ and $\mathrm{d} \tilde{z}$ are null and so $\mathrm{d} z+\mathrm{d} \tilde{z}$ and $I(\mathrm{~d} z+\mathrm{d} \tilde{z})=-\boldsymbol{i}(\mathrm{d} z-\mathrm{d} \tilde{z})$ are orthogonal, closed, divergence-free 1-forms of the same length. Generically, this length will be nonzero on a dense open set, and one can take $\eta_{0}+\boldsymbol{i} \eta_{1}=\mathrm{d} z$ and $\eta_{0}-\boldsymbol{i} \eta_{1}=\mathrm{d} \tilde{z}$. Euclidean reality conditions are easily obtained by setting $\tilde{z}=\bar{z}$ for $I$ real. 
Theorem 7.6. Let $(A, \Phi)$ be a solution to the Hitchin equations (on a trivial spinor-vortex space), with gauge group $\operatorname{Diff}\left(\Sigma^{2}\right)$ for some 2-manifold $\Sigma^{2}$. Then the selfdual space constructed from $(A, \Phi)$ is hypercomplex. Any hypercomplex 4-manifold arises locally in this way, and is hyperkähler if and only if there is a reduction to the gauge group $\operatorname{SDiff}\left(\Sigma^{2}\right)$.

Proof. Choose conformal coordinates $z=x+\boldsymbol{i} y, \tilde{z}=x-\boldsymbol{i} y$ on a trivial spinor-vortex space and write $A=\alpha \mathrm{d} z+\widetilde{\alpha} \mathrm{d} \tilde{z}, \Phi=\phi \mathrm{d} z+\widetilde{\phi} \mathrm{d} \tilde{z}$, where $\alpha, \widetilde{\alpha}, \phi, \widetilde{\phi}$ are complex vector fields on $\Sigma^{2}$. Then the Hitchin equations become

$$
\left[\partial_{z}-\alpha, \widetilde{\phi}\right]=0, \quad\left[\partial_{\tilde{z}}-\widetilde{\alpha}, \phi\right]=0, \quad\left[\partial_{z}-\alpha, \partial_{\tilde{z}}-\widetilde{\alpha}\right]-[\phi, \widetilde{\phi}]=0,
$$

and so the vector fields $V_{0}-i V_{1}=\partial_{z}-\alpha, V_{0}+i V_{1}=\partial_{\tilde{z}}-\widetilde{\alpha}, V_{2}-i V_{3}=\widetilde{\phi}$ and $V_{2}+i V_{3}=\phi$ satisfy equations (7.1)-(7.3). Hence the selfdual space is hypercomplex, with a divergencefree coframe $\left(\eta_{0}, \eta_{1}, \eta_{2}, \eta_{3}\right)$ such that $\eta_{0}+\boldsymbol{i} \eta_{1}=\mathrm{d} z$ and $\eta_{0}-\boldsymbol{i} \eta_{1}=\mathrm{d} \tilde{z}$ so that $\eta_{0}$ and $\eta_{1}$ are closed. Conversely, any hypercomplex 4-manifold admits such a divergence-free coframe, so the distribution generated by $V_{2}$ and $V_{3}$ (i.e., annihilated by $\eta_{0}$ and $\eta_{1}$ ) is integrable. Under this assumption the form of the vector fields given above is entirely general, and so any hypercomplex structure arises in this way.

The characterization of the hyperkähler case is entirely analogous to Theorem 7.3: $V_{i}$ preserve a volume form $e^{-4 f} \mathrm{~d} z \wedge \mathrm{d} \tilde{z} \wedge \eta_{2} \wedge \eta_{3}$ if and only if the area form $e^{-4 f} \eta_{2} \wedge \eta_{3}$ is parallel with respect to the connection $A=\alpha \mathrm{d} z+\widetilde{\alpha} \mathrm{d} \tilde{z}$, and the vector fields $\phi, \widetilde{\phi}$ are area-preserving (on each fibre), which is exactly what it means to have a reduction to $\operatorname{SDiff}\left(\Sigma^{2}\right)$.

Remark 7.7. This result can also be interpreted in Kleinian signature, when the Hitchin equations are replaced by harmonic maps into a Lie group or the principal chiral model. The latter is the context for Husain's formulation [34]. The non-null reduction (in the hyperkähler case) is also discussed briefly by Ward [62] and Ueno [58].

Although this discussion has been local, there are intriguing connections with the global geometry of elliptically fibred K3 surfaces. Yau's solution of the Calabi problem shows that on any K3 surface there is a unique hyperkähler metric in each Kähler class, but no explicit description is known. Any such hyperkähler metric will correspond to a solution of the $\operatorname{SDiff}\left(\Sigma^{2}\right)$ Hitchin equations, once a holomorphic function is chosen on a suitable open subset of the K3 surface to define the dimensional reduction. Now there are K3 surfaces which admit fibrations over $\mathbb{C P}^{1}$ (meromorphic functions) with elliptic curves as fibres, and, generically, 24 singular fibres. On the complement of the singular fibres, there is therefore a dimensional reduction to the Hitchin equations (on $\mathbb{C P}^{1}$ minus 24 points) with gauge group $\operatorname{SDiff}\left(T^{2}\right)$. The work of Gross and Wilson [27] shows that this solution is well approximated by an Abelian solution (gauge group $T^{2}$ ) defining a 'semi-flat' metric.

Remark 7.8. Continuing the development of this section, it is natural to ask if the hypercomplex equations are equivalent to the $\operatorname{Diff}\left(S^{1}\right)$ Bogomolny equation on $\mathbb{R}^{3}$. In fact, it is shown in [8] that the selfdual space constructed from a solution of the $\operatorname{Diff}\left(S^{1}\right)$ generalized Bogomolny equation on an Einstein-Weyl space $B$ is hypercomplex if $B$ is "hyperCR" (see Section 9). However, not all hypercomplex structures arise this way, since for the hyperkähler case in particular, $\operatorname{SDiff}\left(S^{1}\right)=\mathrm{U}(1)$ and only metrics with symmetry are obtained.

Remark 7.9. The Mason-Newman-Dunajski-Joyce construction of Theorem 7.1 also has a gauge-theoretic interpretation, of course: the $V_{i}$ satisfy gauge field equations on a trivial zero-dimensional geometry (!) with gauge group $\operatorname{Diff}\left(\Sigma^{4}\right)$ for some 4 -manifold $\Sigma^{4}$. It has been observed in many places (in particular [40]) that these equations are the reduction of the selfdual Yang-Mills equations on $\mathbb{R}^{4}$ by four translations, and it would therefore be natural to extend the integrable 
background geometries programme to the zero-dimensional case. The background geometry is a four-dimensional conformal vector space $\mathcal{M}$ together with an element $\mathcal{Y}$ of $\wedge_{-}^{2} \mathcal{M}^{*} \otimes \mathcal{M}$ which acts as a right hand side for equations (7.1)-(7.3). The background equation is an unpleasant quadratic condition on $\mathcal{Y}$, which I have left as an exercise for the enthusiastic reader: including all the constructions involving this zero-dimensional geometry would have added unnecessarily to the length of this paper.

\section{Background geometries from gauge fields}

Theorem 6.1 admits the following generalization.

Theorem 8.1. Let $H$ be a transitive group of diffeomorphisms of an $\ell$-manifold $\Sigma^{\ell}$. Suppose that $(\alpha, \varphi)$ is a solution of the gauge field equation on a principal $H$-bundle $P \rightarrow Q$, where $Q$ is a $k$-dimensional background geometry and $k+\ell \leqslant 4$. Then the open subset of $\pi: P \times_{H} \Sigma^{\ell} \rightarrow Q$ where $\widehat{\varphi}$ is surjective carries naturally the structure of a $(k+\ell)$-dimensional background geometry.

Proof. The idea is to apply Theorem 6.1 using the group $H \times \mathbb{R}^{4-k-\ell}$ acting on $\Sigma^{\ell} \times \mathbb{R}^{4-k-\ell}$. Suppose that $\widehat{\varphi}$ is surjective at some point $x$ of the fibre of $P \times_{H} \Sigma^{\ell}$ over $q \in Q$. Let $K \leqslant \mathcal{V}$ be the kernel of $\widehat{\varphi}$ at $x$. Then there exists a solution $\left(\alpha_{0}, \varphi_{0}\right)$ of the gauge field equation, with gauge group $\mathbb{R}^{4-k-\ell}$, defined on a neighbourhood of $q$, such that $\operatorname{ker} \varphi_{0} \cap K=\{0\}$ at $q$ : the linear gauge field equation can be solved with any initial condition. The pair $\left(\widehat{\varphi}, \widehat{\varphi}_{0}\right)$ is therefore an isomorphism on a neighbourhood $M=U \times \mathbb{R}^{4-k-\ell}$ of $\{x\} \times \mathbb{R}^{4-k-\ell}$ in $\left(P \times_{H} \Sigma^{\ell}\right) \times \mathbb{R}^{4-k-\ell}$.

The resulting selfdual conformal structure on $M$ clearly admits $\mathbb{R}^{4-k-\ell}$ as a symmetry group. Hence the quotient $U \subseteq P \times_{H} \Sigma^{\ell}$ is a $(k+\ell)$-dimensional background geometry by the results of Section 2. However, the conformal metric on this quotient is clearly independent of the choice of $\left(\alpha_{0}, \varphi_{0}\right)$, since the pushforward of the inverse metric (6.4) from $\Sigma^{\ell} \times \mathbb{R}^{4-k-\ell}$ to $\Sigma^{\ell}$ kills the components of the vector fields $X_{i}$ in $\mathbb{R}^{4-k-\ell}$. Hence the conformal metric is uniquely defined wherever $\widehat{\varphi}$ is surjective. One also sees that the other fields defining the background geometry are well defined, but the details here depend on the geometry and are rather complicated. The general formulae are given in the following subsections: these will complete the proof, since they are manifestly well defined.

I now give some explicit formulae, using the notation $(A, \Phi)$ for the gauge fields, rather than $(\alpha, \varphi)$. From the above description it is clear at least that the conformal metric on the $(k+\ell)$-dimensional background geometry may be represented contravariantly by

$$
\left(e_{1}-X_{1}\right)^{2}+\cdots+\left(e_{k}-X_{k}\right)^{2}+X_{k+1}^{2}+\cdots+X_{4}^{2},
$$

but note that $X_{k+1}, \ldots X_{4}$ are $4-k$ vector fields on an $\ell$-manifold $\Sigma^{\ell}$ with $\ell \leqslant 4-k$, so inverting this metric is only straightforward when $k+\ell=4$. It will be convenient therefore to introduce a volume form $\nu$ on $\Sigma^{\ell}$ and hence present the explicit formulae in an 'SDiff-gauge'. This will also make it easy to understand the volume-preserving case.

\subsection{Riccati space constructions}

First suppose that $\Phi$ is a generalized Nahm field with values in $\operatorname{Vect}\left(\Sigma^{3}\right)$ and that $\nu$ is a volume form on $\Sigma^{3}$. Then the contravariant metric

$$
\partial_{r}^{2}+\langle\Phi, \Phi\rangle \quad \text { is dual to } \mathrm{d} r^{2}+\langle\eta, \eta\rangle,
$$

where

$$
\eta=\frac{\nu(\Phi \times \Phi, \cdot)}{\nu(\Phi \times \Phi \times \Phi)}
$$


is a section of $\mathcal{E}^{*}$ with values in $\Omega^{1}\left(\Sigma^{3}\right)$ (the space of 1 -forms). Here and in the following $\times$ denotes the cross product $\mathcal{E}^{*} \otimes \mathcal{E}^{*} \rightarrow L^{-1} \mathcal{E}^{*}$ (given by the wedge product and Hodge star operator): the $\operatorname{Vect}\left(\Sigma^{3}\right)$ values of $\Phi$ are then contracted into the entries of the volume form $\nu$. $\mathrm{d} r^{2}+\langle\eta, \eta\rangle$ is the covariant form of the selfdual conformal structure obtained from $\Phi$.

Now suppose that $\Phi$ is a generalized Nahm field with values in $\operatorname{Vect}\left(\Sigma^{2}\right)$ and that $\nu$ is an area form on $\Sigma^{2}$. Let $F$ be an Abelian Nahm field acting on $\mathbb{R}$ with coordinate $\theta$. Then $\Phi+F \partial_{\theta}$ is a generalized Nahm field with values in $\operatorname{Vect}\left(\Sigma^{2} \times \mathbb{R}\right)$ and $\nu \wedge \mathrm{d} \theta$ is a volume form on $\Sigma^{2} \times \mathbb{R}$. The selfdual conformal structure is therefore represented by $\mathrm{d} r^{2}+\langle\eta, \eta\rangle$ where now

$$
\eta=\frac{\nu(\Phi \times \Phi) \mathrm{d} \theta+F \times \nu(\Phi, \cdot)}{\langle F, \nu(\Phi \times \Phi)\rangle} .
$$

Straightforward manipulations and triple cross product identities may be used to rediagonalize the conformal metric

$$
\begin{aligned}
\mathrm{d} r^{2}+\langle\eta, \eta\rangle= & \mathrm{d} r^{2}+\frac{|\nu(\Phi \times \Phi)|^{2} \mathrm{~d} \theta^{2}+2\langle\nu(\Phi \times \Phi), F \times \nu(\Phi, \cdot)\rangle \mathrm{d} \theta+|F \times \nu(\Phi, \cdot)|^{2}}{\langle F, \nu(\Phi \times \Phi)\rangle^{2}} \\
= & \mathrm{d} r^{2}+\frac{|\nu(\Phi \times \Phi)|^{2}|F \times \nu(\Phi, \cdot)|^{2}-\langle\nu(\Phi \times \Phi), F \times \nu(\Phi, \cdot)\rangle^{2}}{|\nu(\Phi \times \Phi)|^{2}\langle F, \nu(\Phi \times \Phi)\rangle^{2}} \\
& +\frac{|\nu(\Phi \times \Phi)|^{2}}{\langle F, \nu(\Phi \times \Phi)\rangle^{2}}\left(\mathrm{~d} \theta+\frac{\langle\nu(\Phi \times \Phi), F \times \nu(\Phi, \cdot)\rangle}{|\nu(\Phi \times \Phi)|^{2}}\right)^{2} \\
= & \mathrm{d} r^{2}+\frac{\langle\nu(\Phi, \cdot), \nu(\Phi, \cdot)\rangle}{|\nu(\Phi \times \Phi)|^{2}}+\frac{|\nu(\Phi \times \Phi)|^{2}}{\langle F, \nu(\Phi \times \Phi)\rangle^{2}}\left(\mathrm{~d} \theta+\frac{\langle\nu(\Phi \times \Phi), F \times \nu(\Phi, \cdot)\rangle}{|\nu(\Phi \times \Phi)|^{2}}\right)^{2} .
\end{aligned}
$$

Hence the conformal structure on the quotient by $\partial_{\theta}$ is represented by the metric

$$
\mathrm{d} r^{2}+\frac{\langle\nu(\Phi, \cdot), \nu(\Phi, \cdot)\rangle}{|\nu(\Phi \times \Phi)|^{2}}
$$

which is, of course, inverse to $\partial_{r}^{2}+\langle\Phi, \Phi\rangle$ : note in particular that $\langle\nu(\Phi \times \Phi), \Phi\rangle=0$, expressing the fact that neither the components of $\Phi$, nor the dual 1-form components of $\nu(\Phi, \cdot)$ are linearly independent (pointwise on $\Sigma^{2}$ ).

I want to give the Weyl structure in an SDiff-gauge, with representative metric

$$
|\nu(\Phi \times \Phi)|^{2} \mathrm{~d} r^{2}+\langle\nu(\Phi, \cdot), \nu(\Phi, \cdot)\rangle .
$$

This metric can be conveniently diagonalized as

$$
g=|\nu(\Phi \times \Phi) \mathrm{d} r+\nu(\Phi, \cdot)|^{2}
$$

using the fact that $\langle\nu(\Phi \times \Phi), \nu(\Phi, \cdot)\rangle=0$. It takes quite a bit of calculation to compute the Jones-Tod Weyl structure $\omega$ in this gauge (i.e., $\left.D=D^{g}+\omega\right)$, but the result is

$$
\omega=\frac{\left\langle 2 \mathcal{B}(\nu(\Phi \times \Phi))-\nu(\Phi \times \Phi) \times \operatorname{div}_{\nu} \Phi, \nu(\Phi \times \Phi) \mathrm{d} r+\nu(\Phi, \cdot)\right\rangle}{|\nu(\Phi \times \Phi)|^{2}}
$$

Writing $\nu=\mathrm{d} p \wedge \mathrm{d} q$ and expanding the cross products in components gives a fuller expression

$$
\begin{aligned}
g & =\eta_{1}^{2}+\eta_{2}^{2}+\eta_{3}^{2}, \\
\omega & =\frac{2 \sum_{j, k} \mathcal{B}_{j k} \nu_{j} \eta_{k}-\sum_{i, j, k} \varepsilon_{i j k} \nu_{i}\left(\phi_{p}^{j}+\psi_{q}^{j}\right) \eta_{k}}{\nu_{1}^{2}+\nu_{2}^{2}+\nu_{3}^{2}},
\end{aligned}
$$


where

$$
\begin{aligned}
& \eta_{i}=\nu_{i} \mathrm{~d} r+\phi^{i} \mathrm{~d} q-\psi^{i} \mathrm{~d} p, \\
& \nu_{1}=\phi^{2} \psi^{3}-\phi^{3} \psi^{2}, \quad \nu_{2}=\phi^{3} \psi^{1}-\phi^{1} \psi^{3}, \quad \nu_{3}=\phi^{1} \psi^{2}-\phi^{2} \psi^{1},
\end{aligned}
$$

and

$$
\Phi=\left(\phi^{1}, \phi^{2}, \phi^{3}\right) \partial_{p}+\left(\psi^{1}, \psi^{2}, \psi^{3}\right) \partial_{q}
$$

Well, nobody said it was going to be easy! The reward is the knowledge that this Weyl structure is Einstein-Weyl if $\mathcal{B}$ satisfies the matrix Riccati equation and $\Phi$ is a generalized Nahm field on this Riccati space.

The construction of spinor-vortex spaces from Riccati spaces is perhaps the most awkward to make explicit, because of the gauge freedom in the bundle $\mathcal{W}$ on a spinor-vortex space. If $\Phi$ is a generalized Nahm field on a Riccati space with values in $\operatorname{Vect}\left(\Sigma^{1}\right)$ for a 1-manifold $\Sigma^{1}$ with coordinate $t$, then the only natural way to proceed is to take $\mathcal{W}$ to be the kernel of $\Phi$ in the pullback of $\mathcal{E}$ to $C \times \Sigma^{1}$. This kernel is not preserved, in general, by the connection $D$ on $\mathcal{E}$, but is preserved by the conformal connection

$$
\nabla=D+\frac{\Phi_{r} \Delta \Phi \mathrm{d} r+\dot{\Phi} \Delta \Phi \mathrm{d} t}{|\Phi|^{2}}
$$

where an affine coordinate $r$ and a $D$-parallel trivialization of $\mathcal{E}$ have been introduced. The complex structure on $\mathcal{W}$ is given by cross product with $\Phi /|\Phi|$, while the holomorphic structure is defined using the connection

$$
\nabla+\frac{\langle\mathcal{B} \Phi, \Phi\rangle \mathrm{id}}{|\Phi|^{2}} \mathrm{~d} r
$$

on $\mathcal{W}$. The other two fields on the spinor-vortex space are

$$
\begin{aligned}
& \mathcal{C}=\mathcal{B}-\frac{\mathcal{B} \Phi \otimes \Phi+\Phi \otimes \mathcal{B} \Phi}{|\Phi|^{2}}+\frac{\langle\mathcal{B} \Phi, \Phi\rangle}{2|\Phi|^{2}}\left(\mathrm{id}+\frac{\Phi \otimes \Phi}{|\Phi|^{2}}\right) \\
& \psi=\frac{\Phi \times(2 \mathcal{B} \Phi+\Phi \times \dot{\Phi})}{|\Phi|^{2}} .
\end{aligned}
$$

These fields satisfy the spinor-vortex equations if $\Phi$ is a generalized Nahm field on a Riccati space. (Since $\Phi /|\Phi|$ is $D$-parallel, it is reasonably straightforward to check (2.6)-(2.7) directly, although (2.8) is harder.)

\subsection{Spinor-vortex space constructions}

The approach here is the same as in the previous subsection, and the details are slightly less complicated. For explicitness, introduce conformal coordinates $z=x+\boldsymbol{i} y, \tilde{z}=x-\boldsymbol{i} y$ on $N$ and let $\nu$ be an area form on $\Sigma^{2}$. First suppose that $(\Phi, \widetilde{\Phi}, \alpha \mathrm{d} z+\widetilde{\alpha} \mathrm{d} \tilde{z})$ is a generalized Hitchin field with values in $\operatorname{Vect}\left(\Sigma^{2}\right)$. Then the contravariant metric

$$
4\left(\partial_{z}-\alpha\right)\left(\partial_{\tilde{z}}-\widetilde{\alpha}\right)+4\langle\Phi, \widetilde{\Phi}\rangle \quad \text { is dual to } \mathrm{d} z \mathrm{~d} \tilde{z}+\langle\eta, \widetilde{\eta}\rangle,
$$

where

$$
\eta=\frac{\nu(\Phi, \cdot)+\nu(\Phi, \alpha) \mathrm{d} z+\nu(\Phi, \widetilde{\alpha}) \mathrm{d} \tilde{z}}{\nu(\Phi, \widetilde{\Phi})} \quad \text { and } \quad \tilde{\eta}=\frac{\nu(\widetilde{\Phi}, \cdot)+\nu(\widetilde{\Phi}, \alpha) \mathrm{d} z+\nu(\widetilde{\Phi}, \widetilde{\alpha}) \mathrm{d} \tilde{z}}{\nu(\widetilde{\Phi}, \Phi)}
$$


Now suppose that $\left(\Phi \otimes \partial_{t}, \widetilde{\Phi} \otimes \partial_{t},(\alpha \mathrm{d} z+\widetilde{\alpha} \mathrm{d} \tilde{z}) \otimes \partial_{t}\right)$ is a generalized Hitchin field with values in $\operatorname{Vect}\left(\Sigma^{1}\right)$ and that $\left(F \partial_{\theta}, \widetilde{F} \partial_{\theta},(\beta \mathrm{d} z+\widetilde{\beta} \mathrm{d} \tilde{z}) \otimes \partial_{\theta}\right)$ is an Abelian Hitchin field acting on $\mathbb{R}$ with coordinate $\theta$. Adding these together produces a generalized Hitchin field with values in $\operatorname{Vect}\left(\Sigma^{1} \times \mathbb{R}\right)$ and $\mathrm{d} t \wedge \mathrm{d} \theta$ is an area form on $\Sigma^{1} \times \mathbb{R}$. Direct substitution gives

$$
\begin{aligned}
& \eta=\frac{F(\mathrm{~d} t+\alpha \mathrm{d} z+\widetilde{\alpha} \mathrm{d} \tilde{z})-\Phi(\mathrm{d} \theta+\beta \mathrm{d} z+\widetilde{\beta} \mathrm{d} \tilde{z})}{F \widetilde{\Phi}-\Phi \widetilde{F}} \\
& \widetilde{\eta}=\frac{\widetilde{F}(\mathrm{~d} t+\alpha \mathrm{d} z+\widetilde{\alpha} \mathrm{d} \tilde{z})-\widetilde{\Phi}(\mathrm{d} \theta+\beta \mathrm{d} z+\widetilde{\beta} \mathrm{d} \tilde{z})}{\widetilde{F} \Phi-\widetilde{\Phi} F} .
\end{aligned}
$$

It is straightforward to rediagonalize $\langle\eta, \widetilde{\eta}\rangle$ to obtain the metric

$$
\begin{aligned}
\mathrm{d} z \mathrm{~d} \tilde{z} & +\frac{(\mathrm{d} t+\alpha \mathrm{d} z+\widetilde{\alpha} \mathrm{d} \tilde{z})^{2}}{4 \Phi \widetilde{\Phi}} \\
& -\frac{\Phi \widetilde{\Phi}}{(F \widetilde{\Phi}-\Phi \widetilde{F})^{2}}\left(\mathrm{~d} \theta+\beta \mathrm{d} z+\widetilde{\beta} \mathrm{d} \tilde{z}-\frac{F \widetilde{\Phi}+\Phi \widetilde{F}}{2 \Phi \widetilde{\Phi}}(\mathrm{d} t+\alpha \mathrm{d} z+\widetilde{\alpha} \mathrm{d} \tilde{z})\right)^{2} .
\end{aligned}
$$

As in the Riccati space construction, the conformal structure is easy to obtain, and is dual to $4\left(\partial_{z}-\alpha\right)\left(\partial_{\tilde{z}}-\widetilde{\alpha}\right)+4 \Phi^{2} \partial_{t}^{2}$, while more work is required to compute the Jones-Tod Weyl structure $\omega$. The result, again in an SDiff-gauge (i.e., the gauge given by $\mathrm{d} t$ ) is reasonably simple, however,

$$
\begin{aligned}
& g=4 \Phi \widetilde{\Phi} \mathrm{d} z \mathrm{~d} \tilde{z}+(\mathrm{d} t+\alpha \mathrm{d} z+\widetilde{\alpha} \mathrm{d} \tilde{z})^{2} \\
& \omega=\left(\dot{\alpha}-\frac{2 \widetilde{\mathcal{C}} \Phi}{\widetilde{\Phi}}\right) \mathrm{d} z+\left(\dot{\widetilde{\alpha}}-\frac{2 \mathcal{C} \widetilde{\Phi}}{\Phi}\right) \mathrm{d} \tilde{z}-\frac{1}{2}\left(\frac{\psi+\dot{\Phi}}{\Phi}+\frac{\widetilde{\psi}+\dot{\widetilde{\Phi}}}{\widetilde{\Phi}}\right)(\mathrm{d} t+\alpha \mathrm{d} z+\widetilde{\alpha} \mathrm{d} \tilde{z}) .
\end{aligned}
$$

This Weyl structure is Einstein-Weyl if $(\Phi, \widetilde{\Phi}, \alpha \mathrm{d} z+\widetilde{\alpha} \mathrm{d} \tilde{z})$ is a generalized Hitchin field on a spinor-vortex space.

\subsection{Einstein-Weyl constructions}

For completeness, I record here the explicit form of the generalized Jones-Tod construction of selfdual spaces from Einstein-Weyl spaces [36, 8]. The conformal structure on $M$ is obtained from the $\operatorname{Diff}\left(\Sigma^{1}\right)$ monopole $(A, \Phi) \partial_{t}$ on $B$ by the formula

$$
\mathrm{c}=\mathrm{c}_{B}+\Phi^{-2}(\mathrm{~d} t+A)^{2},
$$

where $t$ is a coordinate on $\Sigma^{1}$. Compatible metrics for $\mathrm{c}$ are easily obtained by introducing a compatible metric $g_{B}=\mu^{-2} \mathrm{c}_{B}$ on $B$ and writing $\Phi=V \mu^{-1}$. Then $g_{B}+V^{-2}(\mathrm{~d} t+A)^{2}$, $V g_{B}+V^{-1}(\mathrm{~d} t+A)^{2}$ and $V^{2} g_{B}+(\mathrm{d} t+A)^{2}$ are all possibilities. The latter may be written more invariantly as $\Phi^{2} \mathrm{c}_{B}+(\mathrm{d} t+A)^{2}$ : it is the SDiff-gauge determined by $\mathrm{d} t$.

\section{Addendum: null and non-null reductions}

Unlike the preceding addenda, the main observation here is a negative one: the methods of this section do not extend readily to relate nondegenerate background geometries to the $\alpha$ and $\beta$ surface reductions. One might hope to obtain closer links by considering intermediate null reductions, in which the radical of $V M$ is both proper and nontrivial. However, this is beyond the scope of this paper. 


\section{Interlude: the $\operatorname{Diff}(1)$ Hitchin equation}

\section{HyperCR Einstein-Weyl spaces}

An important class of Einstein-Weyl spaces are the hyperCR Einstein-Weyl spaces [22]. In [56], Paul Tod presented a way of reducing the hyperCR Einstein-Weyl equation to a single secondorder differential equation for a complex function of three variables. Since the equation is expected to be integrable, he posed the problem of identifying it. In this section, I will follow a similar approach to Tod and identify the hyperCR Einstein-Weyl equation with the $\operatorname{Diff}\left(S^{1}\right)$ Hitchin equation on a trivial spinor-vortex space.

An Einstein-Weyl structure (c, $D$ ) on $B$ is said to be hyper $C R$ if it admits an orthonormal frame $\chi_{1}, \chi_{2}, \chi_{3}$ for the weightless (co)tangent bundle $L T^{*} B \cong L^{-1} T B$ such that $D \chi_{i}=\kappa * \chi_{i}$ for some section $\kappa$ of $L^{-1}$ and each $i$ - indeed any $\chi$ in the unit sphere generated by $\chi_{1}, \chi_{2}, \chi_{3}$ satisfies the same equation; these $\chi$ 's are called the hyper $C R$ congruences of $B$.

Since the Weyl connection is torsion-free and conformal, it is easy to see that these equations are implied by their skew parts

$$
\begin{aligned}
& \mathrm{d}^{D} \chi_{1}=2 \kappa \chi_{2} \wedge \chi_{3}, \\
& \mathrm{~d}^{D} \chi_{2}=2 \kappa \chi_{3} \wedge \chi_{1}, \\
& \mathrm{~d}^{D} \chi_{3}=2 \kappa \chi_{1} \wedge \chi_{2} .
\end{aligned}
$$

Tod noticed that $\chi_{i}$ satisfying (9.1)-(9.3) determine the Einstein-Weyl space: the conformal metric is $\chi_{1}^{2}+\chi_{2}^{2}+\chi_{3}^{2}$ and the Einstein-Weyl equation follows from (9.1)-(9.3) - see also [10, 22]. Now introduce a gauge $\mu$ with $D \mu=\omega \mu$ and define 1 -forms $\alpha_{i}=2 \mu^{-1} \chi_{i}$. Then the equations (9.1)-(9.3) may be rewritten in the form given by Tod [56]:

$$
\begin{aligned}
& \mathrm{d} \alpha_{1}=-\omega \wedge \alpha_{1}+\kappa \alpha_{2} \wedge \alpha_{3}, \\
& \mathrm{~d} \alpha_{2}=-\omega \wedge \alpha_{2}+\kappa \alpha_{3} \wedge \alpha_{1}, \\
& \mathrm{~d} \alpha_{3}=-\omega \wedge \alpha_{3}+\kappa \alpha_{1} \wedge \alpha_{2} .
\end{aligned}
$$

These equations are easier to interpret after complexification, so that the conformal structure is determined by its null lines, which form a bundle of conics in $P\left(T^{*} B\right)$. This bundle is trivial, since $T^{*} B$ is trivialized by $\alpha_{1}, \alpha_{2}, \alpha_{3}$. The pullback of the tautological 1 -form on $P\left(T^{*} B\right)$ by a constant section is a constant linear combination of $\alpha_{1}, \alpha_{2}$ and $\alpha_{3}$, which is null for a section of the bundle of conics. Then (9.4)-(9.6) are equivalent to the integrability of the distributions defined by these null 1-forms, i.e., to the integrability of a rank 2 distribution $\mathcal{H}$ on the bundle of conics. The integral surfaces are the null surfaces which motivated Cartan [12] to study 3 -dimensional Einstein-Weyl geometry, and the quotient of the bundle of conics by $\mathcal{H}$ is the minitwistor space $\mathcal{S}$ of $B$ [29].

On a general Einstein-Weyl space, $\mathcal{H}$ is defined by the Weyl connection: the hyperCR case is special in that there is a preferred trivialization of the bundle of conics with respect to which the distribution is horizontal. Using this trivialization, the bundle of conics is $B \times \mathbb{P}^{1}$, and after choosing a projective coordinate $\zeta$ on $\mathbb{P}^{1}$, the null 1 -forms are

$$
\alpha_{\zeta}=\alpha_{1}+i \alpha_{2}+2 \zeta \alpha_{3}-\zeta^{2}\left(\alpha_{1}-i \alpha_{2}\right)
$$

The system (9.4)-(9.6) is equivalent to $\alpha_{\zeta} \wedge \mathrm{d} \alpha_{\zeta}=0$ for all $\zeta$. If $X_{1}, X_{2}, X_{3}$ is the dual frame, this means that the vector fields $X_{1}+\boldsymbol{i} X_{2}+\zeta X_{3}$ and $X_{3}-\zeta\left(X_{1}-\boldsymbol{i} X_{2}\right)$ span an integrable distribution (tangent to the null surfaces) for each fixed $\zeta$. This is the hyperCR analogue of the Mason-Newman-Dunajski-Joyce description of hypercomplex structures. 
To see explicitly what this means, put $\varphi=\alpha_{1}+\boldsymbol{i} \alpha_{2}$ and $\omega=-\tau \alpha_{3}+\gamma$, with $\left\langle\gamma, \alpha_{3}\right\rangle=0$, so that (9.4)-(9.6) become

$$
\begin{aligned}
& \mathrm{d} \varphi=\left((\tau+\boldsymbol{i} \kappa) \alpha_{3}-\gamma\right) \wedge \varphi, \\
& \mathrm{d} \alpha_{3}=-\gamma \wedge \alpha_{3}+\frac{i}{2} \kappa \varphi \wedge \bar{\varphi} .
\end{aligned}
$$

The first equation implies the integrability of the distribution defined by $\varphi$. Tod [56] uses (9.7) to put $\varphi=w \mathrm{~d} z$. I will not repeat this here. Instead I want to analyse these equations from the point of view of integrable background geometries. The idea is that the foliation determined by $\chi_{3}$ is a generalized dimensional reduction. To see this, break the equations into horizontal and vertical parts by writing $\alpha_{3}=\mathrm{d} t+A$ for some fibre coordinate $t$ so that $A$ is horizontal (i.e., in the span of $\alpha_{1}$ and $\alpha_{2}$ ). Then for any 1 -form $\beta$,

$$
\mathrm{d} \beta=d_{N} \beta+\mathrm{d} t \wedge \dot{\beta}=d_{N} \beta-A \wedge \dot{\beta}+\alpha_{3} \wedge \dot{\beta},
$$

where $d_{N} \beta$ is a multiple of $\alpha_{1} \wedge \alpha_{2}, \dot{\beta}=\partial_{t} \beta, \alpha_{1}\left(\partial_{t}\right)=0=\alpha_{2}\left(\partial_{t}\right)$, and $\mathrm{d} t\left(\partial_{t}\right)=1$. Hence (9.7)(9.8) become

$$
\begin{aligned}
& d_{N} \varphi-A \wedge \dot{\varphi}=-\gamma \wedge \varphi, \quad \dot{\varphi}=(\tau+i \kappa) \varphi, \\
& d_{N} A-A \wedge \dot{A}=\frac{i}{2} \kappa \varphi \wedge \bar{\varphi}, \quad \dot{A}=\gamma .
\end{aligned}
$$

The equations on the right simply define $\tau+\boldsymbol{i} \kappa$ and $\gamma$, so after computing that $\dot{\varphi} \wedge \bar{\varphi}-\varphi \wedge \dot{\bar{\varphi}}=$ $2 i \kappa \varphi \wedge \bar{\varphi}$, the equations on the left reduce to

$$
\begin{aligned}
& d_{N} \varphi+\dot{A} \wedge \varphi-A \wedge \dot{\varphi}=0, \\
& d_{N} A+\dot{A} \wedge A=\frac{1}{4}(\dot{\varphi} \wedge \bar{\varphi}-\varphi \wedge \dot{\bar{\varphi}}) .
\end{aligned}
$$

The conformal structure on $N$ has representative metric $\varphi \bar{\varphi}$ so the orientation can be chosen so that $\varphi$ has type $(1,0)$. It is now easy to see that these equations are Hitchin's equations with gauge group $\operatorname{Diff}\left(S^{1}\right): \varphi$ and $A$ are 1 -forms on $N$ with values in $\mathfrak{g}=\operatorname{Vect}\left(S^{1}\right) ; \varphi$ is the Higgs field and $A$ is the connection 1-form, satisfying

$$
F^{A}=[\varphi, \bar{\varphi}]_{\mathfrak{g}} \quad \bar{\partial}^{A} \varphi=0 .
$$

These are equivalent to the (9.7)-(9.8) and hence to (9.4)-(9.6): since $\dot{\varphi}$ has type $(1,0)$, one can locally write $\dot{\varphi}=(\tau+\boldsymbol{i} \kappa) \varphi$ and thus define $\alpha_{1}, \alpha_{2}, \alpha_{3}, \omega$.

Theorem 9.1. Let $(A, \varphi)$ be a solution of the $\operatorname{Diff}\left(S^{1}\right)$ Hitchin equations (on a trivial spinorvortex space $N)$. Then the Einstein-Weyl space defined by $(A, \varphi)$ is hyperCR, and one of its hyperCR congruences defines the foliation over $N$. Any hyperCR Einstein-Weyl space arises in this way.

Tod's simplification of the $\operatorname{Diff}\left(S^{1}\right)$ Hitchin equations amounts to a fixing the $\operatorname{Diff}\left(S^{1}\right)$ gauge via $e^{2 i t}=\varphi / \bar{\varphi}$.

In order to obtain new examples of hyperCR Einstein-Weyl spaces, consider Hitchin fields where the gauge group is a finite-dimensional subgroup of $\operatorname{Diff}\left(S^{1}\right)$. The Abelian gauge group U(1) yields only flat Einstein-Weyl spaces, but the affine and projective groups, Aff(R) and $\operatorname{PSL}(2, \mathbb{R})$ are more interesting. The former is more tractable, since $\operatorname{Aff}(\mathbb{R})$ is a solvable group, meaning that the nonlinear Hitchin equations can be solved by integrating a sequence of linear equations. Indeed, writing $\varphi=\varphi_{0}+\varphi_{1} t$ and $A=A_{0}+A_{1} t$ gives

$$
\begin{array}{ll}
d \varphi_{1}=0, & d \varphi_{0}+A_{1} \wedge \varphi_{0}-A_{0} \wedge \varphi_{1}=0, \\
d A_{1}=0, & d A_{0}+A_{1} \wedge A_{0}=\frac{1}{4}\left(\varphi_{1} \wedge \bar{\varphi}_{0}-\varphi_{0} \wedge \bar{\varphi}_{1}\right) .
\end{array}
$$


Locally, the affine gauge freedom can be used to eliminate the linear term $A_{1}$ of the connection $A$, while the conformal gauge freedom can be used to make the linear term $\varphi_{1}$ of the Higgs field $\varphi$ equal to $\lambda \mathrm{d} z$ with $\lambda$ constant (without loss of generality $\lambda=1$ unless it vanishes, which is the Abelian case). Let $\varphi_{0}=i f \mathrm{~d} z$ so that the equations reduce to

$$
\boldsymbol{i} f_{\bar{z}} \mathrm{~d} \bar{z} \wedge \mathrm{d} z=\lambda A_{0} \wedge \mathrm{d} z, \quad d A_{0}=-\frac{1}{2} \boldsymbol{i} f \mathrm{~d} z \wedge \mathrm{d} \bar{z} .
$$

For $\lambda \neq 0$, the general solution up to gauge transformation is therefore determined by a real function $f(z, \bar{z})$ satisfying $\Delta f+2 \lambda^{2} f=0: \varphi=(t+\boldsymbol{i} f) \mathrm{d} z$ and $A=-* \mathrm{~d} f / \lambda$. Hence (local) eigenfunctions of the Laplacian on $\mathbb{R}^{2}$ give rise to hyperCR Einstein-Weyl spaces.

\section{Einstein-Weyl spaces with a geodesic generalized symmetry}

A shear-free geodesic congruence on an Einstein-Weyl space is a weightless unit vector field $\chi \in \mathrm{C}^{\infty}\left(B, L^{-1} T B\right)$ such that

$$
D \chi=\tau(\mathrm{id}-\chi \otimes \chi)+\kappa * \chi
$$

and for sections $\tau, \kappa$ of $L^{-1}$ called the divergence and twist of $\chi$. On a hyperCR EinsteinWeyl space, the hyperCR congruences are examples: they are also divergence-free and in fact this characterizes them. In the previous subsection it was found that the foliation defined by a hyperCR congruence is a generalized symmetry, so it is natural to ask, more generally, when does a shear-free geodesic congruence define a generalized symmetry? Note that the weightless unit vector field tangent to a generalized symmetry over a spinor-vortex space is always shearfree, so this question can be rephrased: when is a generalized symmetry geodesic? Since the Einstein-Weyl space $B$ is completely explicit (8.2) in terms of the Hitchin field on the spinorvortex space $N$, this question is easily answered: the generalized symmetry is geodesic if and only if $\mathcal{C}$ (and $\widetilde{\mathcal{C}}$, which is the complex conjugate in the Euclidean case) vanishes. The Einstein-Weyl structure is then given by

$$
\begin{aligned}
& g=4 \Phi \bar{\Phi} \mathrm{d} z \mathrm{~d} \bar{z}+(\mathrm{d} t+\alpha \mathrm{d} z+\bar{\alpha} \mathrm{d} \bar{z})^{2} \\
& \omega=\dot{\alpha} \mathrm{d} z+\dot{\bar{\alpha}} \mathrm{d} \bar{z}-\frac{1}{2}\left(\frac{\psi+\dot{\Phi}}{\Phi}+\frac{\bar{\psi}+\dot{\bar{\Phi}}}{\bar{\Phi}}\right)(\mathrm{d} t+\alpha \mathrm{d} z+\bar{\alpha} \mathrm{d} \bar{z}) .
\end{aligned}
$$

If $\psi$ vanishes, then the spinor-vortex space is (locally) trivial and this is the case of the previous subsection. Otherwise, $\psi$ is a holomorphic trivialization of $\mathcal{W}$ on the open set where it is nonzero, and the spinor-vortex space is given by a spherical metric on $N$.

Two special cases of this construction have already been studied: the case that $\partial_{t}$ is a genuine symmetry [10], and the case that the congruence is also twist-free [11]. The first class arises by supposing that the gauge group reduces to $\mathrm{U}(1)$ or $\mathbb{R}$ and $B$ is said to be Einstein-Weyl with a geodesic symmetry. The Abelian Hitchin equations are easily solved on the spherical spinor-vortex space yielding the explicit formula

$$
g=|h|^{2} g_{S^{2}}+\beta^{2}, \quad \omega=-\frac{i(h-\bar{h})}{2|h|^{2}} \beta, \quad \mathrm{d} \beta=\frac{1}{2}(h+\bar{h}) \operatorname{vol}_{S^{2}},
$$

where $h$ is a holomorphic function on an open subset of $S^{2}$.

The second class, the hyperCR-Toda spaces, are obtained by from some explicit solutions of the affine Hitchin equations: the twist-free condition reduces the gauge group to $\operatorname{Aff}(\mathbb{R})$; the connection is flat, the linear part of the Higgs field is constant, while the translational part of the Higgs field is given by a holomorphic function $h$. The resulting Einstein-Weyl structure is

$$
g=(t+h)(t+\bar{h}) g_{S^{2}}+\mathrm{d} t^{2}, \quad \omega=-\frac{2 t+h+\bar{h}}{(t+h)(t+\bar{h})} \mathrm{d} t
$$

In this case the twist of the geodesic generalized symmetry $\partial_{t}$ vanishes. 
Note that the general theory of this paper justifies the final remarks of [11], by explaining the sense in which the spherical metric is the natural quotient geometry of both structures (9.10) and (9.11).

In both cases, the Einstein-Weyl space is hyperCR, although the congruence generated by $\partial_{t}$ is no longer one of the hyperCR congruences. The same holds in general.

Theorem 9.2. An Einstein-Weyl space with a geodesic generalized symmetry is hyperCR.

Proof. Gauduchon and Tod [22] show that an Einstein-Weyl space $(B, \mathrm{c}, D)$ is hyperCR with twist $\hat{\kappa}$ if and only if $* D \hat{\kappa}=\frac{1}{2} F^{D}$ and $\hat{\kappa}^{2}=\frac{1}{6} \operatorname{scal}^{D}$.

On an Einstein-Weyl space with a geodesic generalized symmetry, direct computation of $\mathrm{d}^{D} \chi$ in the gauge $(g, \omega)$ for the Einstein-Weyl structure (9.9) yields

$$
\kappa=\frac{1}{4 i}\left(\frac{\dot{\Phi}-\psi}{\Phi}-\frac{\dot{\bar{\Phi}}-\bar{\psi}}{\bar{\Phi}}\right)
$$

Now put

$$
\hat{\kappa}=\frac{1}{2 \boldsymbol{i}}\left(\frac{\dot{\Phi}}{\Phi}-\frac{\dot{\bar{\Phi}}}{\bar{\Phi}}\right)-\kappa=\frac{1}{4 \boldsymbol{i}}\left(\frac{\dot{\Phi}+\psi}{\Phi}-\frac{\dot{\bar{\Phi}}+\bar{\psi}}{\bar{\Phi}}\right) .
$$

In the gauge $(g, \omega)$, the equation $*(\mathrm{~d} \hat{\kappa}-\omega \hat{\kappa})=\frac{1}{2} \mathrm{~d} \omega$ is a straightforward though tedious computation. The equation $\hat{\kappa}^{2}=\frac{1}{6} \mathrm{scal}^{D}$ also follows by direct computation, although the calculation is greatly simplified by using the general formula $-\frac{1}{6}$ scal $^{D}=D_{\chi} \tau+\tau^{2}-\kappa^{2}$ for the scalar curvature of an Einstein-Weyl space with a shear-free geodesic congruence $\chi[10,48]$. In this case $-2 \tau=\psi / \Phi+\bar{\psi} / \bar{\Phi}$ : the verification that $\dot{\tau}-\omega\left(\partial_{t}\right) \tau+\tau^{2}=\kappa^{2}-\hat{\kappa}^{2}$ is now easy.

Conversely, twistor methods show that the foliation defined by any shear-free geodesic congruence on any hyperCR Einstein-Weyl space is a generalized dimensional reduction over a trivial or spherical spinor-vortex geometry, although this is not easy to see by direct computation.

\section{Riccati spaces}

Riccati spaces form a foundation on which higher-dimensional geometries can be built, so although the matrix Riccati equation is easy to solve, it is invaluable to understand the solutions carefully. For this reason, I will begin by tackling the Riccati equation in an invariant way, without choosing a conformal trivialization of $\mathcal{E}$ or a coordinate on $C$. Recall that $\wedge^{3} \mathcal{E}=(T C)^{3}$, and it will be convenient to write $T C=L$, although the choice of orientation implicit in this identification is not essential.

The matrix $\mathcal{B}$ is a section of $L^{-1} \operatorname{Sym}_{0} \mathcal{E}$ and so, at each point of $C$, it has two obvious invariants, of weight -2 and -3 respectively: $x=\frac{2}{3} \operatorname{tr}\left(\mathcal{B}^{2}\right)$ and $y=4 \operatorname{det} \mathcal{B}$, normalized so that the characteristic polynomial of $\mathcal{B}$ is $4 \lambda^{3}-3 x \lambda-y$. The discriminant of this polynomial is the section $y^{2}-x^{3}$ of $L^{-6}$ : more precisely, writing $4\left(y^{2}-x^{3}\right)=-27 c^{2}$ yields $c=\psi_{1} \psi_{2} \psi_{3}$, where $\psi_{1}=\frac{2}{3}\left(\lambda_{2}-\lambda_{3}\right), \psi_{2}=\frac{2}{3}\left(\lambda_{3}-\lambda_{1}\right)$ and $\psi_{3}=\frac{2}{3}\left(\lambda_{1}-\lambda_{2}\right),\left\{\lambda_{i}\right\}$ being the eigenvalues of $\mathcal{B}$; the sign of $c$ depends on the ordering of the eigenvalues. Note that

$$
\psi_{1}+\psi_{2}+\psi_{3}=0, \quad \psi_{1}^{2}+\psi_{2}^{2}+\psi_{3}^{2}=2 x
$$

and

$$
\left(\psi_{1}-\psi_{2}\right)\left(\psi_{2}-\psi_{3}\right)\left(\psi_{3}-\psi_{1}\right)=-8 \lambda_{1} \lambda_{2} \lambda_{3}=-2 y \text {. }
$$




\section{Pencils of conics}

In order to interpret this geometrically, complexify $C$ and $\mathcal{E}$ so that the conformal structure c on $\mathcal{E}$ is determined by its null lines, which form a bundle of conics $\mathcal{S}(\mathcal{E})$ in the bundle $P(\mathcal{E})$ of projective planes over $C$. The role of $\mathcal{B}$ is to determine a pencil of conics in each fibre, associated to the two-dimensional family of bilinear forms $\mathrm{c} \circ(s \mathrm{id}+t \mathcal{B}): \mathrm{c} \circ \mathcal{B}$ is distinguished by being traceless with respect to the fixed bilinear form c. Now, two distinct conics meet in four points, counted with multiplicity, so there are six possible configurations: the generic case (I), where the four points are distinct; the four degenerations (II, III, D, N), when two, three, two pairs, or four points come together; and the trivial case $(0)$, when $\mathcal{B}=0$ and the 'pencil' is constant.

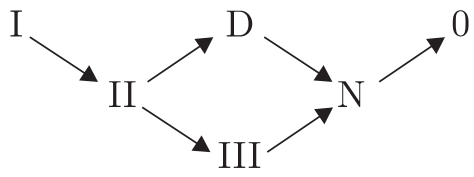

The notation here follows the well-known application of this classification to Weyl tensors in four dimensions. Only types (I, D, 0) are compatible with Euclidean reality conditions: in this case $\mathcal{B}$ must be diagonalizable, and the eigenvalues are either distinct $\left(\lambda_{1}, \lambda_{2}, \lambda_{3}\right)$ with $\lambda_{1}+\lambda_{2}+\lambda_{3}=0$, or of the form $(\lambda, \lambda,-2 \lambda)$, or all zero.

A natural way to analyse a pencil of conics is to identify one of the conics with a projective line $\mathbb{P}^{1}$. In the present situation, this is done by introducing a bundle of spinors $\mathcal{U}$ for $\mathcal{E}$, i.e., with $\mathcal{E}=S^{2} \mathcal{U}$, so that $\mathcal{S}(\mathcal{E})=P(\mathcal{U})$ and $\mathcal{U}$ inherits a connection from $\mathcal{E}$. Trivializing $\mathcal{U}$ using this connection identifies $\mathcal{S}(\mathcal{E})$ locally with $C \times \mathbb{P}^{1}$, on which an affine coordinate $r$ for $C$ and a projective coordinate $\zeta$ for $\mathbb{P}^{1}$ may be introduced. The isomorphism between sections of the inner product bundle $L^{-1} \mathcal{E}$ and vertical vector fields on $\mathcal{S}(\mathcal{E})$ may be described concretely using the induced parallel orthonormal frame for $L^{-1} \mathcal{E}$ : sections induce vector fields via their inner product with the tautological null vector field $e_{\zeta} \otimes \partial_{\zeta}$ where

$$
e_{\zeta}=\left(\frac{1}{2}\left(\zeta^{2}+1\right), \boldsymbol{i} \zeta, \frac{i}{2}\left(\zeta^{2}-1\right)\right) \text {. }
$$

The base locus of the pencil of conics (at each point of $C$ ) consists of the four zeros of the quartic polynomial $\left\langle\mathcal{B}\left(e_{\zeta}\right), e_{\zeta}\right\rangle \otimes \partial_{\zeta}^{2}$, which is a section of $L^{-1} \otimes \mathcal{O}(4)$. It is now straightforward and entirely classical [57] to analyse the zeros of this quartic in the various cases, and hence relate the six types of pencil to properties of the matrix $\mathcal{B}$. In particular, the generic case is given by $c \neq 0$, when the four points are distinct.

Remark 10.1. Four distinct points on $\mathbb{P}^{1}$ are determined up to projective transformation by their cross-ratio, and this freedom is often used to identify the points with $0,1, \infty, t$, with $t$ being the cross-ratio. However, as remarked by Yoshida in his wonderful book [64], "it is not fair that only the fourth point is allowed to move freely". The democratic cross-ratio used there is the point $\left[\psi_{1}, \psi_{2}, \psi_{3}\right]$ on the line $\psi_{1}+\psi_{2}+\psi_{3}=0$ in $\mathbb{P}^{2}$.

\section{Solution of the Riccati equation}

I shall now find the solutions of the Riccati equation $D \mathcal{B}=2\left(\mathcal{B}^{2}\right)_{0}$. Here and elsewhere $D$ denotes differentiation with respect to the affine structure on $C$, and takes a section of a natural bundle $F$ to a section of $L^{-1} F$. In naive terms, $D$ is differentiation with respect to an affine coordinate $r$ (i.e., $\mathrm{d} r$ is a $D$-parallel section of $L^{-1}=T^{*} C$, and $L^{-1}$ is trivialized using this section).

The Riccati equation shows that $D \mathcal{B}$ commutes with $\mathcal{B}$, and so differentiating the CayleyHamilton equation $4 \mathcal{B}^{3}-3 x \mathcal{B}-y$ id $=0$, gives

$$
\begin{aligned}
0 & =12(D \mathcal{B}) \mathcal{B}^{2}-3 x D \mathcal{B}-3 D x \mathcal{B}-D y \mathrm{id}=24 \mathcal{B}^{4}-18 x \mathcal{B}^{2}+3 x^{2} \text { id }-3 D x \mathcal{B}-D y \text { id } \\
& =3(2 y-D x) \mathcal{B}+\left(3 x^{2}-D y\right) \text { id }
\end{aligned}
$$


Hence $D x=2 y, D y=3 x^{2}, D^{2} x=6 x^{2}$ and so $D\left(y^{2}-x^{3}\right)=0$. In other words, the discriminant $c^{2}$ is constant ( $D$-parallel). The constancy of $c$ means that if $\mathcal{B}$ has distinct eigenvalues at a point, it has distinct eigenvalues everywhere, in which case it is diagonalizable, and can be assumed diagonal, since $D \mathcal{B}$ commutes with $\mathcal{B}$. I consider this first.

\section{I. $\mathcal{B}$ has distinct eigenvalues.}

Taking $\mathcal{B}$ diagonal, it is straightforward to compute the equation for the eigenvalues: $D \lambda_{1}=$ $2 \lambda_{1}^{2}-\frac{2}{3}\left(\lambda_{1}^{2}+\lambda_{2}^{2}+\lambda_{3}^{2}\right)$ and similarly for $\lambda_{2}$ and $\lambda_{3}$. Hence $D \psi_{1}=\psi_{1}\left(\psi_{2}-\psi_{3}\right)=-2 \lambda_{1} \psi_{1}$ and similarly for $\psi_{2}$ and $\psi_{3}$. Now let $\chi_{1}=-\psi_{2} \psi_{3}$ etc., so that $\chi_{1} \chi_{2} \chi_{3}=-c^{2}$ and $D \chi_{1}=-\chi_{1}\left(\psi_{2}-\right.$ $\left.\psi_{3}\right)=2 \lambda_{1} \chi_{1}$. Squaring this gives $\left(D \chi_{1}\right)^{2}=\chi_{1}^{2}\left(\psi_{2}^{2}+\psi_{3}^{2}-2 \psi_{2} \psi_{3}\right)=\chi_{1}^{2}\left(\psi_{1}^{2}-4 \psi_{2} \psi_{3}\right)=4 \chi_{1}^{3}+c^{2}$, since $\psi_{1}+\psi_{2}+\psi_{3}=0$. Hence the $\chi_{i}$ all satisfy the equation

$$
(D \chi)^{2}=4 \chi^{3}+c^{2}
$$

which is closely related to the equation for $x$, namely

$$
(D x)^{2}=4 x^{3}-27 c^{2} .
$$

Thus $x$ and the $\chi$ 's are equianharmonic Weierstraß elliptic functions of an affine coordinate, the period lattice for $x$ being the barycentric subdivision of the lattice for the $\chi$ 's.

Remark 10.2. The advantage of an invariant description is the flexibility in the choice of coordinates: one does not have to use the affine coordinate $r$. Indeed, in Section 3, a different gauge choice was motivated: there is a natural projective structure $D^{2}+\frac{1}{6} \operatorname{tr} \mathcal{B}^{2}=D^{2}+\frac{1}{4} x$ and with respect to a projective coordinate $t, D=\partial_{t}+a$, where $\dot{a}-\frac{1}{2} a^{2}=-\frac{1}{2} x$. Now $\dot{x}=2(a x+y)$ and $\dot{y}=3\left(a y+x^{2}\right)$, so that $\ddot{a}=a \dot{a}-\frac{1}{2} \dot{x}=a \dot{a}-a x-y$ and

$$
\begin{aligned}
\dddot{a} & =a \ddot{a}+\dot{a}^{2}-\dot{a} x-\frac{5}{2} a \dot{x}+\left(\frac{3}{2} a \dot{x}-\dot{y}\right)=a \ddot{a}+\dot{a}^{2}-\dot{a}\left(a^{2}-2 \dot{a}\right)-5 a(a \dot{a}-\ddot{a})+3\left(a^{2}-x\right) x \\
& =6 a \ddot{a}+3 \dot{a}^{2}-6 a^{2} \dot{a}+6 \dot{a}\left(a^{2}-2 \dot{a}\right)=6 a \ddot{a}-9 \dot{a}^{2},
\end{aligned}
$$

which is the Chazy equation. Of course it is well-known that the Chazy equation arises in the study of selfdual metrics in a scalar-flat gauge.

The generic solution, given by elliptic functions of the affine coordinate, may instead be presented in terms of modular functions of the projective coordinate $[1,16]$.

I turn next to the degenerate cases, when $c=0$, i.e., $y^{2}=x^{3}=64 \lambda^{6}$, where the eigenvalues of $\mathcal{B}$ are $\lambda, \lambda$ and $-2 \lambda$. Assume first that $\lambda$ is not identically zero. Then, on an open set at least, the (generalized) eigenspaces of $\mathcal{B}$ are constant (again using the fact that $D \mathcal{B}$ commutes with $\mathcal{B}$ ) and hence $\mathcal{B}$ can be assumed to take the the form

$$
\mathcal{B}=\left[\begin{array}{ccc}
\lambda+\mu & \boldsymbol{i} \mu & 0 \\
\boldsymbol{i} \mu & \lambda-\mu & 0 \\
0 & 0 & -2 \lambda
\end{array}\right]
$$

The matrix Riccati equation now yields

$$
D \lambda=-2 \lambda^{2}, \quad D \mu=4 \lambda \mu .
$$

Since $\lambda$ is not identically zero, it is given (up to translation) by $\lambda=1 / 2 r$. This is defined for $r$ nonzero, and $\lambda$ has no zeros. In this coordinate $\mu=b r^{2}$ for constant $b$, which is either identically zero, or nonzero for all nonzero $r$. There are thus two cases.

II. $y^{2}=x^{3}$ is nowhere zero, and $\mathcal{B}$ is nowhere diagonalizable.

D. $y^{2}=x^{3}$ is nowhere zero, and $\mathcal{B}$ is everywhere diagonalizable.

The notation is justified by noting that $\left\langle\mathcal{B}\left(e_{\zeta}\right), e_{\zeta}\right\rangle$ is the quartic $\frac{1}{4}(\zeta-1)^{2}\left(\mu(\zeta-1)^{2}+3 \lambda(\zeta+1)^{2}\right)$ : $\zeta=1$ is a repeated root, as is $\zeta=-1$ when $\mu=0$. 
Remark 10.3. The type (D) solution is even simpler with respect to a projective coordinate. Since $2 \mathcal{B}_{0}^{2}=-2 \lambda \mathcal{B}$, setting $D=\partial_{t}+2 \lambda$ gives $\partial_{t} \mathcal{B}=0$, so that $\lambda$ is constant in this gauge and $a=2 \lambda$ satisfies $\dot{a}-\frac{1}{2} a^{2}=-2 \lambda^{2}=-\frac{1}{3} \operatorname{tr} \mathcal{B}^{2}=-\frac{1}{2} x$; thus $t$ is a projective coordinate with respect to the natural projective structure, and $\mathcal{B}$ and $a$ are constant.

It remains to consider the case that $y^{2}=x^{3}$ is identically zero, i.e., the eigenvalues of $\mathcal{B}$ are all zero, so that $\mathcal{B}^{3}=0$. If $\mathcal{B}^{2}=0$ then $\mathcal{B}$ is constant, so that in any case the kernel and image of $\mathcal{B}$ are constant; $\mathcal{B}$ may thus be assumed to take the form

$$
\mathcal{B}=\left[\begin{array}{ccc}
\mu & \boldsymbol{i} \mu & \beta \\
\boldsymbol{i} \mu & -\mu & \boldsymbol{i} \beta \\
\beta & \boldsymbol{i} \beta & 0
\end{array}\right]
$$

and the matrix Riccati equation yields

$$
D \beta=0, \quad D \mu=2 \beta^{2} .
$$

Hence $\beta$ is a constant, zero if and only if $\mathcal{B}$ is constant, and if $\beta$ is nonzero, then in a suitably translated affine coordinate $\mu=2 \beta^{2} r$. Thus there are three more cases.

III. $\mathcal{B}^{3}=0$ and $\mathcal{B}^{2}$ is nowhere zero.

N. $\mathcal{B}^{2}=0$ and $\mathcal{B}$ is constant and nonzero.

0. $\mathcal{B}=0$.

Again the notation is justified by computing $\left\langle\mathcal{B}\left(e_{\zeta}\right), e_{\zeta}\right\rangle=\frac{1}{4}(\zeta-1)^{3}(\mu(\zeta-1)+2 \boldsymbol{i} \beta(\zeta+1))$.

\section{Isomonodromic deformations}

Recall that, for $\Phi \in \mathrm{C}^{\infty}\left(C, \mathcal{E}^{*} \otimes \mathfrak{g}_{C}\right)$, the generalized Nahm equation (4.4) is

$$
D \Phi-*[\Phi, \Phi]_{\mathfrak{g}}=\mathcal{B} \cdot \Phi \text {. }
$$

I claim that this equation, for $\mathcal{B}$ not identically zero, is equivalent to the fact that

$$
\mathrm{d}+\frac{\Phi}{\mathcal{B}}=\mathrm{d}+\frac{\Phi\left(e_{\zeta}\right) \mathrm{d} \zeta}{\left\langle\mathcal{B}\left(e_{\zeta}\right), e_{\zeta}\right\rangle}
$$

is an isomonodromic family of connections on $\mathbb{C P}^{1}$ parameterized by $C$, with four poles along the base locus of the pencil of conics, where I use the tautological null vector field $e_{\zeta}(10.1)$ to clarify the meaning of the connection 1-form. More precisely, this follows from the fact that the meromorphic connection

$$
\mathrm{d}+\frac{\Phi+\langle * \mathcal{B}, \Phi\rangle}{\mathcal{B}}=\mathrm{d}+\frac{\Phi\left(e_{\zeta}\right) \mathrm{d} \zeta+\left\langle * e_{\zeta} \wedge \mathcal{B}\left(e_{\zeta}\right), \Phi\right\rangle \mathrm{d} r}{\left\langle\mathcal{B}\left(e_{\zeta}\right), e_{\zeta}\right\rangle}
$$

defined on the pullback of $\mathfrak{g}_{C}$ to the bundle of conics $\mathcal{S}(\mathcal{E})$ over the Riccati space $C$, is flat if and only if $\Phi$ satisfies the generalized Nahm equation.

This is a straightforward verification. Equivalently, if the gauge algebra $\mathfrak{g}_{C}$ is represented as a Lie algebra of vector fields, then this observation can be reformulated as the integrability of a rank two distribution on a bundle over $\mathcal{S}(\mathcal{E})$, and the poles of the connection can be viewed as points of $\mathcal{S}(\mathcal{E})$ over which the distribution is tangent to the fibres. Explicitly, in the above coordinates, two vector fields generating this distribution are

$$
\begin{aligned}
& \left\langle\mathcal{B}\left(e_{\zeta}\right), e_{\zeta}\right\rangle \partial_{\zeta}+\Phi\left(e_{\zeta}\right), \\
& \partial_{r}+\left\langle\mathcal{B}\left(e_{\zeta}\right), e_{\zeta}^{\prime}\right\rangle \partial_{\zeta}+\Phi\left(e_{\zeta}^{\prime}\right),
\end{aligned}
$$

where $e_{\zeta}^{\prime}=(\zeta, \boldsymbol{i}, \boldsymbol{i} \zeta)$. One can easily check that the distribution is closed under Lie bracket.

An advantage of this vector field interpretation is that it also makes sense on the trivial Riccati space $\mathcal{B}=0$, when (10.2) reduces to the standard Lax pair for the Nahm equation. 


\section{Addendum: Riccati spaces and integrability by the method of hydrodynamic reductions}

In [74], Ferapontov et al. independently discover the integrability of a system of equations equivalent to the generalized Nahm equations on Riccati spaces. Their study concerns equations of the form

$$
\frac{\partial^{2}\left(A^{\alpha \beta}(u)\right)}{\partial x^{\alpha} \partial x^{\beta}}=0
$$

where $u=u\left(x^{1}, x^{2}, x^{3}\right)$ is a function of three variables, and $A^{\alpha \beta}$ is a $3 \times 3$ symmetric matrix of functions of one variable. (Here I use the summation convention on Greek indices; this may also be viewed as abstract index notation.) Expanding one derivative yields the equivalent formulation

$$
\frac{\partial}{\partial x^{\alpha}}\left(V^{\alpha \beta}(u) \frac{\partial u}{\partial x^{\beta}}\right)=0
$$

where $V^{\alpha \beta}$ is the derivative of $A^{\alpha \beta}$ (i.e., $V=A^{\prime}$ ).

The approach in [74] characterizes integrability using the method of hydrodynamic reductions; this tests for the existence of sufficiently many multi-phase solutions $u=U\left(R^{1}, R^{2}, \ldots R^{N}\right)$, where the $R^{j}\left(x^{1}, x^{2}, x^{3}\right)$ are solutions to arbitrarily many commuting $(1+1)$-dimensional systems of hydrodynamic type. The result of this analysis is that (10.3) is integrable in this sense if and only if there is a scalar function $k(u)$ of one variable such that

$$
V^{\prime \prime}(u)=(\log \operatorname{det} V)^{\prime}(u) V^{\prime}(u)+k(u) V(u)
$$

or equivalently $\left(V^{\prime} / \operatorname{det}(V)\right)^{\prime}=k V$.

The relation to the Riccati space equation is subtle, and involves introducing a $u$-dependent triple of vectors $\theta_{\alpha}^{i}(u)(i=1,2,3)$ such that

$$
V=(\operatorname{det} \theta) \theta^{-1}\left(\theta^{\mathrm{T}}\right)^{-1}, \quad \text { i.e., } \quad V^{\alpha \beta}=\sum_{i=1}^{3}(\operatorname{det} \theta)\left(\theta^{-1}\right)_{i}^{\alpha}\left(\theta^{-1}\right)_{i}^{\beta},
$$

where $\theta=\left(\theta_{\alpha}^{i}\right)$ is assumed invertible. Now write $\mathcal{C}_{i j}:=\left(\theta^{-1}\right)_{i}^{\alpha}\left(\theta_{\alpha}^{j}\right)^{\prime}$ so that $\theta_{\alpha}^{i}=\sum_{j=1}^{3} \mathcal{C}_{i j} \theta_{\alpha}^{j}$. There is a gauge freedom in the choice of $\theta$, which may be used to suppose $\mathcal{C}$ is symmetric.

Substituting (10.5) into (10.4), straightforward computation, using the symmetry of $\mathcal{C}$ together with standard matrix identities such as $\left(A^{-1}\right)^{\prime}=-A^{-1} A^{\prime} A^{-1}$ and $(\operatorname{det} A)^{-1}(\operatorname{det} A)^{\prime}=$ $\operatorname{tr}\left(A^{-1} A^{\prime}\right)$, then yields

$$
\mathcal{C}^{\prime}=2 \mathcal{C}^{2}-(\operatorname{tr} \mathcal{C}) \mathcal{C}+c I,
$$

where $c$ is an unknown scalar function of $u$ (related to $k$ ).

Now decompose $\mathcal{C}=\mathcal{B}+a I$ with $\mathcal{B}$ tracefree; then the trace part of the above equation determines $c$, while the trace-free part is the Riccati equation for $\mathcal{B}$. Thus, remarkably, the hydrodynamic integrability condition agrees with the twistor-theoretic Riccati equation.

After classifying solutions of (10.4) (equivalently, solutions of the Riccati equation), Ferapontov et al. compute the form of the equation (10.3) for $u$ arising from each solution. They then observe that the central quadric Ansatz

$$
x^{\alpha} M_{\alpha \beta}(u) x^{\beta}=1
$$

for $u$ yields all Painlevé equations [74]. 
It is this geometry that originally led to the identification of (10.4) with the Riccati equation. The key observation is that (10.3) is a divergence form equation, meaning that $u$ is in the kernel of the Laplace-Beltrami operator of the metric $g_{\alpha \beta}(u)$ with $g=\operatorname{adj}(V)=(\operatorname{det} V) V^{-1}$ so that $V=\sqrt{\operatorname{det} g} g^{-1}$. Hence the equations in this class have the form

$$
\mathrm{d} *{ }_{g} \mathrm{~d} u=0,
$$

where $g=g(u)$, and $*_{g}$ is the associated Hodge star operator. Now $\theta_{\alpha}^{i}(u)$ has an interpretation, as a framing of the metric $g$ :

$$
g_{\alpha \beta}(u)=\sum_{i=1}^{3} \theta_{\alpha}^{i}(u) \theta_{\beta}^{i}(u) .
$$

In terms of the geometry of $g, \theta$ is thus an $\mathbb{R}^{3}$-valued 1-form: on vector fields $X, Y$, the metric is $g(u)(X, Y)=\langle\theta(u) X, \theta(u) Y\rangle$. Thus there is a gauge freedom to rotate $\theta$ by an $\mathrm{SO}(3)$-valued function of $u$ : this is the freedom used to make $\mathcal{C}_{i j}$ symmetric above (which in turn fixes $\theta$ up to a rigid rotation).

As will be explained in Section 11.2 (the last part of the next and final interlude), (10.3) is a hodograph transformation of the generalized Nahm equation with gauge group $\operatorname{SDiff}\left(\Sigma^{2}\right)$. The central quadric Ansatz corresponds to a reduction of gauge group from $\operatorname{SDiff}\left(\Sigma^{2}\right)$ to $\mathrm{SU}(2)$ (which acts by area preserving diffeomorphisms on the 2-sphere). This in turn explains the appearance of the Painlevé equations: generalized Nahm equations with gauge group $G$ describe isomonodromic deformation problems for $G$-connections on $\mathbb{C} P^{1}$ with four poles, and, when $G=\mathrm{SU}(2)$ (rank 2 bundles over $\mathbb{C} P^{1}$ ), the connection between such isomonodromy problems and the Painlevé equations is well known [35].

\section{Interlude: the $\operatorname{Diff}(2)$ generalized Nahm equation}

This interlude is devoted to Weyl structures of the form (8.1). It follows from the work of Section 8, that these Weyl structures are Einstein-Weyl provided that $\mathcal{B}$ satisfies the matrix Riccati equation and $\Phi^{i}=\phi^{i} \partial_{p}+\psi^{i} \partial_{q}(i=1,2,3)$ defines a generalized Nahm field on this Riccati space, the gauge group being a subgroup of $\operatorname{Diff}\left(\Sigma^{2}\right)$ where $\Sigma^{2}$ is a surface with coordinates $p$, $q$.

However, as details were omitted in Section 8, I present a self-contained study of these Weyl structures in the following two cases:

(i) $\mathcal{B}=0$ (so the Riccati space is trivial);

(ii) the $\Phi^{i}$ are area preserving vector fields.

These examples have an interest that stretches beyond the proscenium of Einstein-Weyl geometry, since they are closely related both to well-known integrable systems, and also to hypercomplex, hyperkähler and scalar-flat Kähler structures in four dimensions.

\subsection{HyperCR Einstein-Weyl spaces and the Diff(2) Nahm equation}

In Section 9, hyperCR Einstein-Weyl spaces were related to the $\operatorname{Diff}\left(S^{1}\right)$ Hitchin equation. Now it is known $[8,22]$ that any solution of the $\operatorname{Diff}\left(S^{1}\right)$ Einstein-Weyl Bogomolny equation on a hyperCR Einstein-Weyl space gives rise to a hypercomplex 4-manifold (which is sometimes hyperkähler).

This two step construction ties in with the construction of hypercomplex and hyperkähler structures from the $\operatorname{Diff}\left(\Sigma^{2}\right)$ Hitchin equation: see Section 7. On the other hand, also in Section 7 , hypercomplex and hyperkähler structures were related to the $\operatorname{Diff}\left(\Sigma^{3}\right)$ Nahm equation, 
so it is natural to expect that hyperCR Einstein-Weyl spaces may be constructed from the $\operatorname{Diff}\left(\Sigma^{2}\right)$ Nahm equation.

In fact this is not hard to see. Suppose that $D=D^{g}+\omega$ is a Weyl structure given by

$$
g=\eta_{1}^{2}+\eta_{2}^{2}+\eta_{3}^{2}, \quad \omega=-\frac{\sum_{i, j, k} \varepsilon_{i j k} \nu_{i}\left(\phi_{p}^{j}+\psi_{q}^{j}\right) \eta_{k}}{\nu_{1}^{2}+\nu_{2}^{2}+\nu_{3}^{2}}
$$

where

$$
\begin{aligned}
& \eta_{i}=\nu_{i} \mathrm{~d} r+\phi^{i} \mathrm{~d} q-\psi^{i} \mathrm{~d} p, \\
& \nu_{1}=\phi^{2} \psi^{3}-\phi^{3} \psi^{2}, \quad \nu_{2}=\phi^{3} \psi^{1}-\phi^{1} \psi^{3}, \quad \nu_{3}=\phi^{1} \psi^{2}-\phi^{2} \psi^{1},
\end{aligned}
$$

and

$$
\Phi=\left(\phi^{1}, \phi^{2}, \phi^{3}\right) \partial_{p}+\left(\psi^{1}, \psi^{2}, \psi^{3}\right) \partial_{q} .
$$

The Nahm equation expands to give

$$
\phi_{r}^{1}=\phi^{2} \phi_{p}^{3}-\phi^{3} \phi_{p}^{2}+\psi^{2} \phi_{q}^{3}-\psi^{3} \phi_{q}^{2}, \quad \psi_{r}^{1}=\phi^{2} \psi_{p}^{3}-\phi^{3} \psi_{p}^{2}+\psi^{2} \psi_{q}^{3}-\psi^{3} \psi_{q}^{2}
$$

and cyclic permutations. From this it follows easily that $\mathrm{d} \eta_{1}+\omega \wedge \eta_{1}=\kappa \eta_{2} \wedge \eta_{3}$ (and similarly for the cyclic permutations), where

$$
\kappa=\frac{\nu_{1}\left(\phi_{p}^{1}+\psi_{q}^{1}\right)+\nu_{2}\left(\phi_{p}^{2}+\psi_{q}^{2}\right)+\nu_{3}\left(\phi_{p}^{3}+\psi_{q}^{3}\right)}{\nu_{1}^{2}+\nu_{2}^{2}+\nu_{3}^{2}} .
$$

Hence the Weyl structure is hyperCR Einstein-Weyl. Note that if the Nahm field preserves the (a priori arbitrary) area form $\mathrm{d} p \wedge \mathrm{d} q$ then $\kappa=0$ and the Einstein-Weyl space is flat. Ward [60] uses this fact to linearize the Nahm equation in this case.

In fact any hyperCR Einstein-Weyl space $B$ arises locally from this construction. One way to see this is to choose an Abelian monopole on $B$. This defines a hypercomplex structure with a triholomorphic vector field $K$ tangent to the fibres over $B$. For example, $\kappa$ is an Abelian monopole on any hyperCR Einstein-Weyl space and the corresponding hypercomplex structure is hyperkähler with a triholomorphic homothetic vector field [22].

Now let $r$ be a solution of $\Delta r=0$, which is constant on the fibres over $B$, where $\Delta$ is the Laplacian of the Obata connection. This defines a divergence-free coframe $\mathrm{d} r, I \mathrm{~d} r, J \mathrm{~d} r, K \mathrm{~d} r$ and a dual frame of vector fields $V_{0}, V_{1}, V_{2}, V_{3}$. Since $r$ is constant on the fibres over $B, K$ is tangent to the level surfaces of $r$, so it is in the span of $V_{1}, V_{2}, V_{3}$ and commutes with them all (since it is triholomorphic). Therefore the gauge group of the Nahm field defined by $V_{1}, V_{2}, V_{3}$ reduces to the group of diffeomorphisms of $\Sigma^{3}$ commuting with the flow of $K$. Taking the local quotient by $K$ gives a Nahm field with gauge group $\operatorname{Diff}\left(\Sigma^{2}\right)$ for some surface $\Sigma^{2}$, and this is clearly a Nahm field giving rise to the Einstein-Weyl space $B$.

Theorem 11.1. Let $\Phi$ be a solution of the Nahm equation with gauge group $\operatorname{Diff}\left(\Sigma^{2}\right)$ for some surface $\Sigma^{2}$. Then the Einstein-Weyl space defined by $\Phi$ is hyperCR, and any hyperCR EinsteinWeyl space arises locally in this way. If there is a reduction to $\operatorname{SDiff}\left(\Sigma^{2}\right)$, the Einstein-Weyl space is flat.

There is a great deal more freedom in this construction than in the $\operatorname{Diff}\left(S^{1}\right)$ Hitchin construction of hyperCR Einstein-Weyl spaces, which can be an advantage or a drawback, since the same hyperCR Einstein-Weyl space will arise in many ways. There are some interesting special cases one could consider, such as the group $\mathrm{SL}(2, \mathbb{C})$ acting on $\mathbb{C P}^{1}$ (which does not preserve an area form). The Nahm equations in this case reduce to a (complexified) Euler top equation, solvable in terms of elliptic functions. 


\subsection{The $\operatorname{SDiff}(2)$ generalized Nahm equation}

In addition to hyperCR Einstein-Weyl spaces, another important class consists of the EinsteinWeyl spaces arising from solutions of the $\mathrm{SU}(\infty)$ Toda field equation $u_{x x}+u_{y y}+\left(e^{u}\right)_{z z}=0$ $[39,55,60]$. The terminology originates by regarding the equation as a dispersionless limit of the $\mathrm{SU}(N)$ Toda equation as $N \rightarrow \infty$ : the Toda equation is a two-dimensional system with independent variables $(x, y)$; the $z$ variable is normally discrete, but becomes continuous in the limit. The large $N$ limit of $\mathrm{SU}(N)$ may be interpreted as the group $\operatorname{SDiff}\left(S^{1} \times S^{1}\right)$ of area preserving diffeomorphisms of a torus, with its Lie algebra $\mathbb{Z}$-graded by the Fourier components in one of the circles. There is a potential source of confusion here, however, since the $\mathrm{SU}(\infty)$ Toda equation is related to area preserving diffeomorphisms in another way. Namely, each solution gives rise to a hyperkähler metric with a Killing vector $K[6]$, and hyperkähler metrics are in turn obtained from the $\operatorname{SDiff}\left(\Sigma^{2}\right)$ Hitchin equations on $\mathbb{R}^{2}$, as shown in Theorem 7.6 (cf. [62]). Hence one expects the $\mathrm{SU}(\infty)$ Toda equation to be equivalent to a symmetry reduction of the $\operatorname{SDiff}\left(\Sigma^{2}\right)$ Hitchin equations to one dimension.

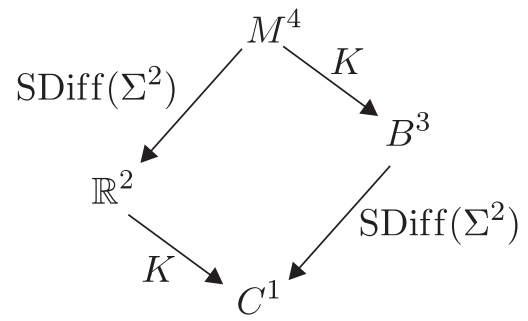

The Einstein-Weyl structure on a Toda Einstein-Weyl space $B^{3}$ is given by the metric and Weyl 1-form

$$
h=e^{u}\left(\mathrm{~d} x^{2}+\mathrm{d} y^{2}\right)+\mathrm{d} z^{2}, \quad \omega_{h}=-u_{z} \mathrm{~d} z .
$$

In these geometric terms, the $\mathrm{SU}(\infty)$ Toda equation may be written $\mathrm{d} *_{h} \mathrm{~d} u=0$, and so $*_{h} \mathrm{~d} u$ is a closed 2-form, which is therefore locally of the form $\mathrm{d} p \wedge \mathrm{d} q$. Now $(p, q, u)$ will be functionally independent unless $0=\mathrm{d} u \wedge{ }^{h} \mathrm{~d} u=*_{h}|\mathrm{~d} u|^{2}$. Hence (in Euclidean signature), $(p, q, u)$ may be used as coordinates, except in the trivial case ( $u$ constant). The solution of the Toda equation is now given implicitly by the functions $(x, y, z)$ of $(p, q, u)$. These functions will turn out to satisfy a generalized Nahm equation on the type (D) Riccati space with gauge group $\operatorname{SDiff}\left(\Sigma^{2}\right)$.

In order to see this, and relate it to the work of Ferapontov et al. [74], consider the generalized Nahm equation on any Riccati space with affine coordinate $r$, where the Higgs fields $\Phi^{i}=$ $\phi^{i} \partial_{p}+\psi^{i} \partial_{q}$ preserve the area form $\mathrm{d} p \wedge \mathrm{d} q$ on a surface $\Sigma^{2}$ with coordinates $(p, q)$. Motivated by the general construction of Section 8 , introduce the Weyl structure

$$
g=\eta_{1}^{2}+\eta_{2}^{2}+\eta_{3}^{2}, \quad \omega=2 \frac{\lambda_{1} \nu_{1} \eta_{1}+\lambda_{2} \nu_{2} \eta_{2}+\lambda_{3} \nu_{3} \eta_{3}}{\nu_{1}^{2}+\nu_{2}^{2}+\nu_{3}^{2}}
$$

where

$$
\begin{aligned}
& \eta_{i}=\nu_{i} \mathrm{~d} r+\phi^{i} \mathrm{~d} q-\psi^{i} \mathrm{~d} p, \\
& \nu_{1}=\phi^{2} \psi^{3}-\phi^{3} \psi^{2}, \quad \nu_{2}=\phi^{3} \psi^{1}-\phi^{1} \psi^{3}, \quad \nu_{3}=\phi^{1} \psi^{2}-\phi^{2} \psi^{1} .
\end{aligned}
$$

The metric $g$ can be simplified considerably using the fact that area preserving vector fields are locally hamiltonian: this means one can write $\phi^{i}=F^{i}$ and $\psi^{i}=-F_{p}^{i}$ for some functions $F^{1}, F^{2}, F^{3}$ of $(p, q, r)$. The (r-dependent) constants of integration for the hamiltonians may be chosen so that the $F^{i}$ satisfy the generalized Nahm equation in the Lie algebra of functions 
under Poisson bracket: $F^{1}{ }_{r}-\left\{F^{2}, F^{3}\right\}=\sum_{j} \mathcal{B}_{1 j} F^{j}$ and so on. (The system $p^{i}{ }_{r}-\sum_{j} \mathcal{B}_{i j} p^{i}=q^{i}$ has local solutions for any $q^{i}(r)$, and these can be added to $F^{i}$. However, if the $\Phi^{i}$ take values in a Lie subalgebra of the area preserving vector fields, then the $F^{i}$ may take values in a central extension of this Lie subalgebra.) The metric now reduces to

$$
g=\sum_{i}\left(\mathrm{~d} F^{i}-\sum_{j} \mathcal{B}_{i j} F^{j} \mathrm{~d} r\right)^{2}=\sum_{i} \theta_{\alpha}^{i}(r) \theta_{\beta}^{i}(r) \mathrm{d} x^{\alpha} \mathrm{d} x^{\beta},
$$

where $\theta_{\alpha}^{i}$ is a basis of solutions to the linear system $\theta_{r}^{i}=\sum_{j} \mathcal{B}_{i j} \theta^{j}$ and, using the summation convention on Greek indices, $F^{i}=\theta_{\alpha}^{i} x^{\alpha}$, for some functions $x^{\alpha}(p, q, r)(\alpha=1,2,3)$. The equation $F_{r}^{i}-\frac{1}{2} \sum_{j, k} \varepsilon_{i j k}\left\{F^{j}, F^{k}\right\}=\sum_{j} \mathcal{B}_{i j} F^{j}$ is immediately equivalent to

$$
\theta_{\alpha}^{i} x_{r}^{\alpha}=\sum_{j, k} \varepsilon_{i j k} \theta_{\alpha}^{j} \theta_{\beta}^{k}\left\{x^{\alpha}, x^{\beta}\right\}
$$

Now for any functions $x^{\alpha}(p, q, r)$ direct calculations yield the general jacobian identities

$$
\begin{aligned}
& \left\{x^{2}, x^{3}\right\} \mathrm{d} x^{1}+\left\{x^{3}, x^{1}\right\} \mathrm{d} x^{2}+\left\{x^{1}, x^{2}\right\} \mathrm{d} x^{3}=J(x) \mathrm{d} r, \\
& x^{1}{ }_{r} \mathrm{~d} x^{2} \wedge \mathrm{d} x^{3}+x^{2}{ }_{r} \mathrm{~d} x^{3} \wedge \mathrm{d} x^{1}+x^{3}{ }_{r} \mathrm{~d} x^{1} \wedge \mathrm{d} x^{2}=J(x) \mathrm{d} p \wedge \mathrm{d} q,
\end{aligned}
$$

where $J(x)$ is the determinant of the jacobian of $\left(x^{1}, x^{2}, x^{3}\right)$ with respect to $(p, q, r)$. Hence (11.1) holds if and only if $*_{g} \mathrm{~d} r=\mathrm{d} p \wedge \mathrm{d} q$.

This argument was carried out using an affine coordinate $r$. However, any coordinate $t$ can be used, simply by writing $\partial_{t}=\partial_{r}+a$. The following result summarizes the general construction.

Proposition 11.2. Consider the 3-dimensional metric

$$
g=\sum_{i}\left(\theta_{\alpha}^{i}(t) \mathrm{d} x^{\alpha}\right)^{2}
$$

where $\theta_{\alpha}^{i}$ is a basis of solutions of the linear system $\theta^{i}{ }_{t}-a \theta^{i}=\sum_{j} \mathcal{B}_{i j} \theta^{j}$ for some symmetric traceless matrix $\mathcal{B}_{i j}(t)$, and $x^{\alpha}(p, q, t)$ are arbitrary functions. Then $*_{g} \mathrm{~d} t=\mathrm{d} p \wedge \mathrm{d} q$ if and only if the functions $F^{i}=\theta_{\alpha}^{i} x^{\alpha}$ satisfy

$$
F_{t}^{i}-a F^{i}-\frac{1}{2} \sum_{j, k} \varepsilon_{i j k}\left\{F^{j}, F^{k}\right\}=\sum_{j} \mathcal{B}_{i j} F^{j} .
$$

Remark 11.3. We pause briefly to reiterate (conversely) the link between this analysis and the work of Ferapontov et al. [74]. The framing $\theta_{\alpha}^{i}$ of the metric $g$ appearing in $\mathrm{d} *_{g} \mathrm{~d} u=0$, i.e., the formulation (10.6) of (10.3), provides a diagonalization

$$
g_{\alpha \beta} \mathrm{d} x^{\alpha} \mathrm{d} x^{\beta}=\sum_{i=1}^{3}\left(\theta_{\alpha}^{i} \mathrm{~d} x^{\alpha}\right)^{2}=\sum_{i=1}^{3}\left(\mathrm{~d} F^{i}-(\mathcal{C} F)^{i} \mathrm{~d} u\right)^{2},
$$

where $F^{i}=\theta_{\alpha}^{i} x^{\alpha},(\mathcal{C} F)^{i}=\sum_{i=1}^{3} \mathcal{C}_{i j} F^{j}$. Writing $*_{g} \mathrm{~d} u=\mathrm{d} p \wedge \mathrm{d} q$ yields

$$
\left(F^{i}\right)^{\prime}-\frac{1}{2} \sum_{i, j, k} \varepsilon_{i j k}\left\{F^{j}, F^{k}\right\}=\sum_{j} \mathcal{C}_{i j} F^{j},
$$

which reduces to the generalized Nahm equation after splitting $\mathcal{C}$ into its tracefree and tracelike parts. 
Returning to the $\mathrm{SU}(\infty)$ Toda equation, $\left(\theta_{\alpha}^{i}\right)$ is now a diagonal matrix with eigenvalues $\left(e^{u / 2}, e^{u / 2}, 1\right)$. The case that $u$ is constant (so that $\mathcal{B}=0$ ) is the overlap with the previous subsection, and this construction recovers Ward's linearization of the $\mathrm{SU}(\infty)$ Nahm equation [61]. On the other hand if $u$ is not constant, $t=u$ can be used as a coordinate, so that $a=1 / 3$ and $\mathcal{B}_{i j}$ is diagonal with constant eigenvalues $1 / 6,1 / 6,-1 / 3$. This is the type (D) solution of the matrix Riccati equation with $\lambda=1 / 6$ (see Remark 10.3).

There is another class of Einstein-Weyl spaces, governed by the dKP equation [18]. The Weyl structure in this case is Lorentzian:

$$
h=\mathrm{d} y^{2}-4 \mathrm{~d} x \mathrm{~d} t-4 u \mathrm{~d} t^{2}, \quad \omega_{h}=2 u_{x} \mathrm{~d} t .
$$

The Einstein-Weyl condition may again be written $\mathrm{d} *_{h} \mathrm{~d} u=0$, which now reduces to the dKP equation $u_{y y}=\left(u_{t}-u u_{x}\right)_{x}$. If the solutions $\left(\theta_{\alpha}^{i}\right)$ are taken to be $(1+u, 2 \boldsymbol{i} u, 0),(2 \boldsymbol{i} u, 1-u, 0)$ and $(0,0,1)$, then $h=\sum_{i, \alpha, \beta} \theta_{\alpha}^{i}(r) \theta_{\beta}^{i}(r) \mathrm{d} x^{\alpha} \mathrm{d} x^{\beta}$ with $-\sqrt{2} t=x^{1}+\boldsymbol{i} x^{2}, 2 \sqrt{2} x=x^{1}-\boldsymbol{i} x^{2}, y=x^{3}$, whereas $\mathcal{B}$ is the type $(\mathrm{N})$ solution of the Riccati equation. The equivalence works as long as $|\mathrm{d} u|_{h} \neq 0$.

Theorem 11.4. The $\mathrm{SU}(\infty)$ Toda and dKP equations are generically locally equivalent to $\operatorname{SDiff}\left(\Sigma^{2}\right)$ generalized Nahm equations, on the type (D) and (N) Riccati space respectively.

The equivalence in each case is obtained by a "hodograph transformation", i.e., dependent and independent variables are exchanged. The beauty of such transformations is that they interchange coordinate-invariance and gauge-invariance, which is why they are potentially useful for studying geometric differential equations, where the independent variables are often not well defined.

This result was first obtained in for the $\mathrm{SU}(\infty)$ Toda equation and type (D) Nahm equation. The extension to more general Nahm equations resulted from discussions with Maciej Dunajski. Together with Paul Tod, he has given a detailed discussion of the dKP case [20].

Solutions of the $\mathrm{SU}(\infty)$ Toda equation may be divided into two classes according to whether they are most easily presented explicitly or implicitly: in the former class, there are very few examples - to the best of my knowledge, the only examples are the separable solutions [6, 23] and the hyperCR-Toda solutions [11] (the latter were discussed in Section 9).

The class of implicit solutions is much richer and all of them (that I know of) are obtained by the above hodograph transformation. There are two general classes.

\section{Solutions with a Killing vector $[10,60]$}

Suppose that the generalized Nahm field is invariant under a one-dimensional symmetry group of $\Sigma^{2}$, generated by a hamiltonian vector field $X$, with momentum map $\eta$, and let $\psi$ be a function on $\Sigma^{2}$ with $\mathrm{d} \psi(X)=1$. Then $\psi, \eta$ can be used as coordinates on $\Sigma^{2}, X=\partial_{\psi}$ and the area form is $\mathrm{d} \psi \wedge \mathrm{d} \eta$. The Lie algebra of hamiltonian vector fields commuting with $\partial_{\psi}$ consists of vector fields of the form $b \partial_{\eta}+W(\eta) \partial_{\psi}$, where $b$ is constant (on $\Sigma^{2}$ ), and the hamiltonian is $-b \psi+V(\eta$ ) where $V_{\eta}=W$. The solutions found by Ward [60] are given by the metric

$$
h=\rho^{2}\left(\mathrm{~d}\left(U_{\eta}\right)^{2}+\mathrm{d} \psi^{2}\right)+\mathrm{d}\left(\rho U_{\rho}\right)^{2},
$$

where $U(\rho, \eta)$ satisfies $\left(\rho U_{\rho}\right)_{\rho}+\rho U_{\eta \eta}=0$, the equation for axisymmetric harmonic functions on $\mathbb{R}^{3}$. In this case one of the hamiltonians is simply $\psi$. The general solutions were found in [10] following similar ideas. 


\section{Solutions constant on central ellipsoids [55]}

Motivated by the Pedersen-Poon Ansatz for scalar-flat Kähler metrics [47], Tod [55] observed that solutions of the Toda equation which are constant on central ellipsoids may be obtained from an ordinary differential equation, namely Painlevé's third equation. Painlevé III is equivalent to the isomonodromic deformation problem for an $\mathrm{SU}(2)$ connection with two double poles, and hence to the generalized Nahm equation with gauge group $\mathrm{SU}(2)$ on the type (D) Riccati space. Tod's solutions are obtained by reducing to the gauge algebra $\mathfrak{s o}(3)$ of infinitesimal isometries of $S^{2}$. Solutions constant on central ellipsoids arise in this way because ellipsoids are precisely the quotients of left invariant metrics on $S^{3}=\mathrm{SU}(2)$ by a U(1) subgroup.

Similar reductions are known in the dKP case - see $[18,20]$ - and the latter construction is the context for the central quadric Ansatz and Painlevé equations of [74].

\section{Further speculations}

The integrable background geometries discussed in this paper are those arising from the selfduality equation for conformal structures in four dimensions. Roughly speaking, they are characterized as geometries associated to a twistor space containing rational curves with degree 2 normal bundle, although this requires some interpretation for the one or two-dimensional geometries, where the twistor space is not Hausdorff and has dimension zero or one respectively.

Null reductions lead to twistor spaces with lower degree normal bundles. For instance, a surface with a projective structure may be viewed as a moduli space of rational curves in a complex surface with normal bundle $\mathcal{O}(1)$ (see Hitchin [32] and LeBrun [38]).

These considerations lead to the idea of extending the integrable background geometry concept to higher degree normal bundles. This would encompass the Einstein-Weyl and selfdual hierarchies (where the normal bundle is $\mathcal{O}(n)$ or $\mathcal{O}(n) \otimes \mathbb{C}^{2}$ respectively), as well as quaternionic geometry (normal bundle $\mathcal{O}(1) \otimes \mathbb{C}^{2 k}$ ).

In the language of integrable systems, the concept of a background geometry appears to be related to Lax systems involving a derivative with respect to the spectral parameter $\zeta$. The zeros of the coefficient of $\partial_{\zeta}$ are associated with poles of the Lax system: the prototype here is the one-dimensional case (isomonodromic deformations). In its most general form, the background geometry idea might be regarded as a study of these extra $\partial_{\zeta}$ terms: general principles for the introduction of such terms have been developed by Burtsev-Zakharov-Mikhailov [7]. At the very least, it is desirable to know when these terms can be eliminated. For the geometries studied in this paper, this question has an answer: they can be eliminated provided that the background geometry is a trivial Riccati space, a trivial or spherical spinor-vortex space, a hyperCR EinsteinWeyl space, or a hypercomplex selfdual space.

Returning to the general context of this paper, recent work of Ferapontov and Kruglikov [75] suggest deep links between selfduality (hence twistor theory) and integrability in low dimensions, going beyond the inspirational analysis by Mason and Woodhouse. For example, in the particular case of equations of the form (10.6), the Einstein-Weyl structure may be recovered from the linearization $\mathrm{d} *_{g(u)} \mathrm{d} v+\mathrm{d}\left(v *_{g}{ }^{\prime}(u) \mathrm{d} u\right)=0$. The leading term in $v$ is

$$
g^{\alpha \beta}(u) \frac{\partial^{2} v}{\partial x^{\alpha} \partial x^{\beta}}
$$

so the symbol of the linearized equation is the inverse metric $g^{-1}$ (up to a conformal factor) of the Einstein-Weyl structure. Ferapontov and Kruglikov also show how to recover (the 1-form of) the Weyl structure. They obtain a similar result for several classes of PDEs, relating the integrability of such equations (by the method of hydrodynamic reductions) to Einstein-Weyl or selfdual conformal structures on their moduli spaces. 


\section{A Table of integrable background geometries}

The following table summarizes the background equations and gauge field equations in each dimension (given in Euclidean signature for convenience).

In this table, I indicate the data defining the background geometry, starting with the conformal manifold $Q$ and the conformal vector bundle $\mathcal{V}=L, \mathcal{W}$, or $\mathcal{E}$ (which has rank 1,2, or 3 respectively) followed by the other data: $D$ is a conformal connection on $\mathcal{V}, \bar{\partial}^{a}$ a holomorphic structure, whereas $\mathcal{C}, \psi, \mathcal{B}$ are sections of $\mathcal{W}^{*} \otimes_{\mathbb{C}} \mathcal{W}^{*} \otimes_{\mathbb{C}} T N, \mathcal{W}^{*} \otimes_{\mathbb{C}} T N$ and $T^{*} C \otimes \operatorname{Sym}_{0} \mathcal{E}$ respectively.

The gauge field equations are for a pair $(A, \Phi)$, where $A$ is a $G$-connection and the Higgs field $\Phi$ is an $\mathfrak{g}_{Q}$-valued section of $\mathcal{V}^{*}$, except that in four dimensions there is no Higgs field, and in one dimension, the connection $A$ can be assumed trivial.

\begin{tabular}{|l|c|c|c|}
\hline Dimension & Data & Background equations & Gauge field equations \\
\hline 4 Selfdual & $M$ & $W^{-}=0$ & $* F^{A}=F^{A}$ \\
\hline 3 Einstein-Weyl & $(B, L), D$ & $r_{0}^{D}=0$ & $* D^{A} \Phi=F^{A}$ \\
\hline 2 Spinor-vortex & $(N, \mathcal{W})$, & $\bar{\partial}^{a} \mathcal{C}=0$, \\
& $\left(\bar{\partial}^{a}, \mathcal{C}, \psi\right)$ & $\bar{\partial}^{a} \psi=-3 \mathcal{C} \bar{\psi}$, \\
& $s_{\mathcal{W}}^{-1} T N=\psi \bar{\psi}-2 \mathcal{C} \overline{\mathcal{C}}$ & $F^{A}-[\Phi, \bar{\Phi}]_{\mathfrak{g}}=\psi \wedge \bar{\Phi}+\bar{\psi} \wedge \Phi$, \\
& $\bar{\partial}^{a, A} \Phi=\mathcal{C} \Phi$ \\
\hline Riccati & $(C, \mathcal{E}),(D, \mathcal{B})$ & $D \mathcal{B}=2\left(\mathcal{B}^{2}\right)_{0}$. & $D^{A} \Phi-*[\Phi, \Phi]_{\mathfrak{g}}=\mathcal{B} \cdot \Phi$ \\
\hline
\end{tabular}

Finally, I summarize the background geometry constructions in an $\operatorname{SDiff}(\Sigma)$-gauge, i.e., using an arbitrarily chosen volume form $\nu$ on $\Sigma\left(\nu=\mathrm{d} t\right.$ on $\left.\Sigma^{1}\right)$.

\section{Selfdual spaces}

- From Einstein-Weyl spaces:

$$
g=\Phi^{2} \mathrm{c}_{B}+(\mathrm{d} t+A)^{2} .
$$

- From spinor-vortex spaces (with conformal coordinate $z$ ):

$$
g=|\nu(\Phi, \bar{\Phi})|^{2} \mathrm{~d} z \mathrm{~d} \bar{z}+\langle\nu(\Phi, \cdot)+\nu(\Phi, A), \nu(\bar{\Phi}, \cdot)+\nu(\bar{\Phi}, A)\rangle .
$$

- From Riccati spaces (with affine coordinate $r$ ):

$$
g=|\nu(\Phi \times \Phi \times \Phi)|^{2} \mathrm{~d} r^{2}+|\nu(\Phi \times \Phi, \cdot)|^{2} .
$$

\section{Einstein-Weyl spaces}

- From spinor-vortex spaces (with conformal coordinate $z$ ):

$$
\begin{aligned}
& g=4 \Phi \bar{\Phi} \mathrm{d} z \mathrm{~d} \bar{z}+(\mathrm{d} t+\alpha \mathrm{d} z+\bar{\alpha} \mathrm{d} \bar{z})^{2} \\
& \omega=\dot{A}-\frac{2 \overline{\mathcal{C}} \Phi}{\bar{\Phi}} \mathrm{d} z-\frac{2 \mathcal{C} \Phi}{\Phi} \mathrm{d} \bar{z}-\frac{1}{2}\left(\frac{\psi+\dot{\Phi}}{\Phi}+\frac{\bar{\psi}+\dot{\bar{\Phi}}}{\bar{\Phi}}\right)(\mathrm{d} t+\alpha \mathrm{d} z+\bar{\alpha} \mathrm{d} \bar{z}) .
\end{aligned}
$$

- From Riccati spaces (with affine coordinate $r$ ):

$$
\begin{aligned}
& g=|\nu(\Phi \times \Phi) \mathrm{d} r+\nu(\Phi, \cdot)|^{2} \\
& \omega=\frac{\left\langle 2 \mathcal{B}(\nu(\Phi \times \Phi))-\nu(\Phi \times \Phi) \times \operatorname{div}_{\nu} \Phi, \nu(\Phi \times \Phi) \mathrm{d} r+\nu(\Phi, \cdot)\right\rangle}{|\nu(\Phi \times \Phi)|^{2}} .
\end{aligned}
$$




\section{Spinor-vortex spaces from Riccati spaces}

$\mathcal{W}$ is the kernel of $\Phi$ in the pullback of $\mathcal{E}$ to $C \times \Sigma^{1}$ with complex structure given by cross product with $\Phi /|\Phi|$ and holomorphic structure induced by the conformal connection

$$
D+\frac{\left(\Phi_{r} \triangle \Phi+\langle\mathcal{B} \Phi, \Phi\rangle \mathrm{id}\right) \mathrm{d} r+\dot{\Phi} \triangle \Phi \mathrm{d} t}{|\Phi|^{2}}
$$

the conformal structure is represented by $g=|\Phi|^{2} \mathrm{~d} r^{2}+\mathrm{d} t^{2}$ and the other two fields are

$$
\begin{aligned}
& \mathcal{C}=\mathcal{B}-\frac{\mathcal{B} \Phi \otimes \Phi+\Phi \otimes \mathcal{B} \Phi}{|\Phi|^{2}}+\frac{\langle\mathcal{B} \Phi, \Phi\rangle}{2|\Phi|^{2}}\left(\mathrm{id}+\frac{\Phi \otimes \Phi}{|\Phi|^{2}}\right) \\
& \psi=\frac{\Phi \times(2 \mathcal{B} \Phi+\Phi \times \dot{\Phi})}{|\Phi|^{2}} .
\end{aligned}
$$

\section{Acknowledgements}

I would like to thank Harry Braden, Maciej Dunajski, Evgeny Ferapontov, Paul Gauduchon, Nigel Hitchin, Boris Kruglikov, Claude LeBrun, Lionel Mason, Ian Strachan, Paul Tod and Nick Woodhouse for helpful discussions. This work was supported by the EPSRC, the Leverhulme Trust, and the William Gordon Seggie Brown Trust.

\section{References}

[1] Ablowitz M.J., Clarkson P.A., Solitons, nonlinear evolution equations and inverse scattering, London Mathematical Society Lecture Note Series, Vol. 149, Cambridge University Press, Cambridge, 1991.

[2] Ashtekar A., Jacobson T., Smolin L., A new characterization of half-flat solutions to Einstein's equation, Comm. Math. Phys. 115 (1988), 631-648.

[3] Atiyah M., Hitchin N., The geometry and dynamics of magnetic monopoles, M.B. Porter Lectures, Princeton University Press, Princeton, NJ, 1988.

[4] Atiyah M.F., Hitchin N.J., Singer I.M., Self-duality in four-dimensional Riemannian geometry, Proc. Roy. Soc. London Ser. A 362 (1978), 425-461.

[5] Atiyah M.F., Ward R.S., Instantons and algebraic geometry, Comm. Math. Phys. 55 (1977), 117-124.

[6] Boyer C.P., Finley J.D., Killing vectors in self-dual, Euclidean Einstein spaces, J. Math. Phys. 23 (1982), $1126-1130$.

[7] Burtsev S.P., Zakharov V.E., Mikhailov A.V., Inverse scattering method with variable spectral parameter, Theoret. and Math. Phys. 70 (1987), 227-240.

[8] Calderbank D.M.J., Selfdual Einstein metrics and conformal submersions, math.DG/0001041.

[9] Calderbank D.M.J., Mason L.J., Spinor-vortex geometry and microtwistor theory, unpublished, 2002.

[10] Calderbank D.M.J., Pedersen H., Selfdual spaces with complex structures, Einstein-Weyl geometry and geodesics, Ann. Inst. Fourier (Grenoble) 50 (2000), 921-963, math.DG/9911117.

[11] Calderbank D.M.J., Tod P., Einstein metrics, hypercomplex structures and the Toda field equation, Differential Geom. Appl. 14 (2001), 199-208, math.DG/9911121.

[12] Cartan É., Sur une classe d'espaces de Weyl, Ann. Sci. École Norm. Sup. (3) 60 (1943), 1-16.

[13] Chakravarty S., Mason L., Newman E.T., Canonical structures on anti-self-dual four-manifolds and the diffeomorphism group, J. Math. Phys. 32 (1991), 1458-1464.

[14] Dancer A.S., Scalar-flat Kähler metrics with SU(2) symmetry, J. Reine Angew. Math. 479 (1996), 99-120.

[15] Dancer A.S., Strachan I.A.B., Cohomogeneity-one Kähler metrics, in Twistor Theory (Plymouth), Lecture Notes in Pure and Appl. Math., Vol. 169, Dekker, New York, 1995, 9-27.

[16] Dubrovin B., Geometry of 2D topological field theories, in Integrable Systems and Quantum Groups (Montecatini Terme, 1993), Lecture Notes in Math., Vol. 1620, Springer, Berlin, 1996, 120-348, hep-th/9407018. 
[17] Dunajski M., The twisted photon associated to hyper-Hermitian four-manifolds, J. Geom. Phys. 30 (1999), 266-281, math.DG/9808137.

[18] Dunajski M., Mason L.J., Tod P., Einstein-Weyl geometry, the dKP equation and twistor theory, J. Geom. Phys. 37 (2001), 63-93, math.DG/0004031.

[19] Dunajski M., Mason L.J., Woodhouse N.M.J., From 2D integrable systems to self-dual gravity, J. Phys. A: Math. Gen. 31 (1998), 6019-6028, solv-int/9809006.

[20] Dunajski M., Tod P., Einstein-Weyl spaces and dispersionless Kadomtsev-Petviashvili equation from Painlevé I and II, Phys. Lett. A 303 (2002), 253-264, nlin.SI/0204043.

[21] Gauduchon P., Structures de Weyl-Einstein, espaces de twisteurs et variétés de type $S^{1} \times S^{3}$, J. Reine Angew. Math. 469 (1995), 1-50.

[22] Gauduchon P., Tod K.P., Hyper-Hermitian metrics with symmetry, J. Geom. Phys. 25 (1998), 291-304.

[23] Gegenberg J.D., Das A., Stationary Riemannian space-times with self-dual curvature, Gen. Relativity Gravitation 16 (1984), 817-829.

[24] Gibbons G.W., Hawking S.W., Gravitational multi-instantons, Phys. Lett. B 78 (1978), 430-432.

[25] Glazebrook J.F., Kamber F.W., Pedersen H., Swann A., Foliation reduction and self-duality, in Geometric Study of Foliations (Tokyo, 1993), Editors T. Mizutani, K. Masuda, S. Matsumoto, T. Inaba, T. Tsuboi, Y. Mitsumatsu, World Sci. Publ., River Edge, NJ, 1994, 219-249.

[26] Grant J.D.E., Strachan I.A.B., Hypercomplex integrable systems, Nonlinearity 12 (1999), 1247-1261, solv-int/9808019.

[27] Gross M., Wilson P.M.H., Large complex structure limits of $K 3$ surfaces, J. Differential Geom. 55 (2000), 475-546, math.DG/0008018.

[28] Hashimoto Y., Yasui Y., Miyagi S., Ootsuka T., Applications of the Ashtekar gravity to four-dimensional hyper-Kähler geometry and Yang-Mills instantons, J. Math. Phys. 38 (1997), 5833-5839, hep-th/9610069.

[29] Hitchin N.J., Complex manifolds and Einstein's equations, in Twistor Geometry and Nonlinear Systems (Primorsko, 1980), Lecture Notes in Math., Vol. 970, Editors H.D. Doebner, T.D. Palev, Springer, Berlin, 1982, 73-99.

[30] Hitchin N.J., The self-duality equations on a Riemann surface, Proc. London Math. Soc. 55 (1987), 59-126.

[31] Hitchin N.J., Twistor spaces, Einstein metrics and isomonodromic deformations, J. Differential Geom. 42 (1995), 30-112.

[32] Hitchin N.J., Geometrical aspects of Schlesinger's equation, J. Geom. Phys. 23 (1997), 287-300.

[33] Hitchin N.J., Hypercomplex manifolds and the space of framings, in The Geometric Universe (Oxford, 1996), Editors S.A. Huggett, L.J. Mason, K.P. Tod, S.T. Tsou, N.M.J. Woodhouse, Oxford University Press, Oxford, 1998, 9-30.

[34] Husain V., Self-dual gravity as a two-dimensional theory and conservation laws, Classical Quantum Gravity 11 (1994), 927-937, gr-qc/9310003.

[35] Jimbo M., Miwa T., Ueno K., Monodromy preserving deformation of linear ordinary differential equations with rational coefficients. I. General theory and $\tau$-function, Phys. D 2 (1981), 306-352.

[36] Jones P.E., Tod K.P., Minitwistor spaces and Einstein-Weyl spaces, Classical Quantum Gravity 2 (1985), $565-577$.

[37] Joyce D.D., Explicit construction of self-dual 4-manifolds, Duke Math. J. 77 (1995), 519-552.

[38] LeBrun C., Spaces of complex geodesics and related structures, Ph.D. Thesis, University of Oxford, 1980.

[39] LeBrun C., Explicit self-dual metrics on $\mathbb{C P}_{2} \# \cdots \# \mathbb{C P}_{2}$, J. Differential Geom. 34 (1991), 223-253.

[40] Mason L.J., Newman E.T., A connection between the Einstein and Yang-Mills equations, Comm. Math. Phys. 121 (1989), 659-668.

[41] Mason L.J., Woodhouse N.M.J., Integrability, self-duality, and twistor theory, London Mathematical Society Monographs. New Series, Vol. 15, The Clarendon Press, Oxford University Press, New York, 1996.

[42] Maszczyk R., The classification of self-dual Bianchi metrics, Classical Quantum Gravity 13 (1996), 513-527.

[43] Maszczyk R., Mason L.J., Woodhouse N.M.J., Self-dual Bianchi metrics and the Painlevé transcendents, Classical Quantum Gravity 11 (1994), 65-71.

[44] Nahm W., The construction of all self-dual multimonopoles by the ADHM method, in Monopoles in Quantum Field Theory (Trieste, 1981), World Sci. Publishing, Singapore, 1982, 87-94. 
[45] Obata M., Affine connections on manifolds with almost complex, quaternion or Hermitian structure, Jpn. J. Math. 26 (1956), 43-77.

[46] Park Q.H., Self-dual gravity as a large- $N$ limit of the 2D nonlinear sigma model, Phys. Lett. B 238 (1990), 287-290.

[47] Pedersen H., Poon Y.S., Kähler surfaces with zero scalar curvature, Classical Quantum Gravity 7 (1990), 1707-1719.

[48] Pedersen H., Tod K.P., Three-dimensional Einstein-Weyl geometry, Adv. Math. 97 (1993), 74-109.

[49] Penrose R., Nonlinear gravitons and curved twistor theory, Gen. Relativity Gravitation 7 (1976), 31-52.

[50] Plebański J.F., Some solutions of complex Einstein equations, J. Math. Phys. 16 (1975), 2395-2402.

[51] Tafel J., Two-dimensional reductions of the self-dual Yang-Mills equations in self-dual spaces, J. Math. Phys. 34 (1993), 1892-1907.

[52] Tafel J., Wójcik D., Null Killing vectors and reductions of the self-duality equations, Nonlinearity 11 (1998), 835-844.

[53] Tod K.P., Self-dual Einstein metrics from the Painlevé VI equation, Phys. Lett. A 190 (1994), $221-224$.

[54] Tod K.P., Cohomogeneity-one metrics with self-dual Weyl tensor, in Twistor Theory (Plymouth), Lecture Notes in Pure and Appl. Math., Vol. 169, Editor S.A. Huggett, Dekker, New York, 1995, 171-184.

[55] Tod K.P., Scalar-flat Kähler and hyper-Kähler metrics from Painlevé-III, Classical Quantum Gravity 12 (1995), 1535-1547.

[56] Tod K.P., 'Special' Einstein-Weyl spaces, Twistor Newsletter 42 (1997), 13-15.

[57] Todd J.A., Projective and analytical geometry, Pitman Publishing Corporation, New York, 1946.

[58] Ueno T., Integrable field theories derived from 4-D self-dual gravity, Modern Phys. Lett. A 11 (1996), 545-552, hep-th/9508012.

[59] Ward R.S., Integrable and solvable systems, and relations among them, Philos. Trans. Roy. Soc. London Ser. A 315 (1985), 451-457.

[60] Ward R.S., Einstein-Weyl spaces and SU( $\infty$ ) Toda fields, Classical Quantum Gravity 7 (1990), L95-L98.

[61] Ward R.S., Linearization of the SU( $\infty)$ Nahm equations, Phys. Lett. B 234 (1990), 81-84.

[62] Ward R.S., The SU( $\infty)$ chiral model and self-dual vacuum spaces, Classical Quantum Gravity 7 (1990), L217-L222.

[63] Weyl H., Space, time, matter, Dover, New York, 1952.

[64] Yoshida M., Hypergeometric functions, my love (modular interpretations of configuration spaces), Aspects of Mathematics, Vol. E32, Friedr. Vieweg \& Sohn, Braunschweig, 1997.

\section{Additional references}

[65] Calderbank D.M.J., Selfdual 4-manifolds, projective structures, and the Dunajski-West construction, SIGMA 10 (2014), 035, 18 pages, math.DG/0606754.

[66] Donaldson S., Fine J., Toric anti-self-dual 4-manifolds via complex geometry, Math. Ann. 336 (2006), 281309, math.DG/0602423.

[67] Dunajski M., Harmonic functions, central quadrics and twistor theory, Classical Quantum Gravity 20 (2003), 3427-3440, math.DG/0303181.

[68] Dunajski M., Solitons, instantons, and twistors, Oxford Graduate Texts in Mathematics, Vol. 19, Oxford University Press, Oxford, 2010.

[69] Dunajski M., Grant J.D.E., Strachan I.A.B., Multidimensional integrable systems and deformations of Lie algebra homomorphisms, J. Math. Phys. 48 (2007), 093502, 11 pages, nlin.SI/0702040.

[70] Dunajski M., Krynski W., Einstein-Weyl geometry, dispersionless Hirota equation and Veronese webs, Math. Proc. Cambridge Philos. Soc., to appear, arXiv:1301.0621.

[71] Dunajski M., Sparling G., A dispersionless integrable system associated to Diff $\left(S^{1}\right)$ gauge theory, Phys. Lett. A 343 (2005), 129-132, nlin.SI/0503030.

[72] Dunajski M., West S., Anti-self-dual conformal structures with null Killing vectors from projective structures, Comm. Math. Phys. 272 (2007), 85-118, math.DG/0601419. 
[73] Dunajski M., West S., Anti-self-dual conformal structures in neutral signature, in Recent Developments in Pseudo-Riemannian Geometry, Editors D.V. Alekseevsky, H. Baum, ESI Lect. Math. Phys., Eur. Math. Soc., Zürich, 2008, 113-148, math.DG/0610280.

[74] Ferapontov E.V., Huard B., Zhang A., On the central quadric ansatz: integrable models and Painlevé reductions, J. Phys. A: Math. Theor. 45 (2012), 195204, 11 pages, arXiv:1201.5061.

[75] Ferapontov E.V., Kruglikov B., Dispersionless integrable systems in 3D and Einstein-Weyl geometry, arXiv:1208.2728.

[76] Fine J., Toric anti-self-dual Einstein metrics via complex geometry, Math. Ann. 340 (2008), 143-157, math.DG/0609487.

[77] LeBrun C., Mason L.J., Zoll manifolds and complex surfaces, J. Differential Geom. 61 (2002), 453-535, math.DG/0211021.

[78] LeBrun C., Mason L.J., Nonlinear gravitons, null geodesics, and holomorphic disks, Duke Math. J. 136 (2007), 205-273, math.DG/0504582.

[79] LeBrun C., Mason L.J., The Einstein-Weyl equations, scattering maps, and holomorphic disks, Math. Res. Lett. 16 (2009), 291-301, arXiv:0806.3761.

[80] LeBrun C., Mason L.J., Zoll metrics, branched covers, and holomorphic disks, Comm. Anal. Geom. 18 (2010), 475-502, arXiv:1002.2993.

[81] Nakata F., Self-dual Zollfrei conformal structures with $\alpha$-surface foliation, J. Geom. Phys. 57 (2007), 20772097, math.DG/0701116.

[82] Nakata F., Singular self-dual Zollfrei metrics and twistor correspondence, J. Geom. Phys. 57 (2007), 14771498, math.DG/0607276.

[83] Nakata F., A construction of Einstein-Weyl spaces via LeBrun-Mason type twistor correspondence, Comm. Math. Phys. 289 (2009), 663-699, arXiv:0806.2696. 